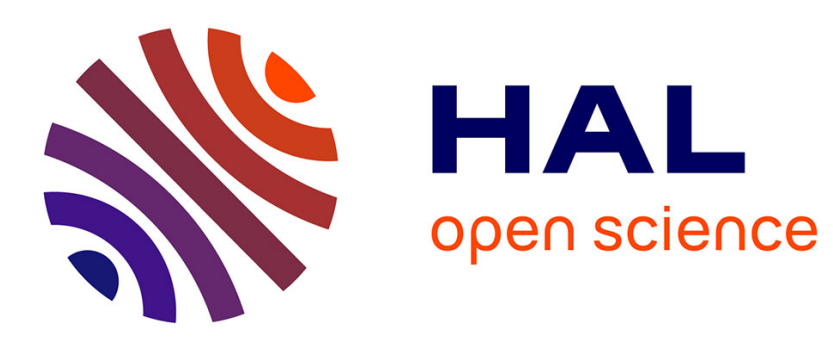

\title{
La partie méridionale du premier village du quartier de l'Île à Martigues (Bouches-du-Rhône) entre le milieu du Ves. et la fin du IIIe s. av. J.-C.
}

Axel Cauvin

\section{- To cite this version:}

Axel Cauvin. La partie méridionale du premier village du quartier de l'île à Martigues (Bouches-du-Rhône) entre le milieu du Ves. et la fin du IIIe s. av. J.-C.. Documents d'archéologie méridionale, 2017, 39. halshs-01686092

\section{HAL Id: halshs-01686092 \\ https://shs.hal.science/halshs-01686092}

Submitted on 9 Dec 2019

HAL is a multi-disciplinary open access archive for the deposit and dissemination of scientific research documents, whether they are published or not. The documents may come from teaching and research institutions in France or abroad, or from public or private research centers.
L'archive ouverte pluridisciplinaire HAL, est destinée au dépôt et à la diffusion de documents scientifiques de niveau recherche, publiés ou non, émanant des établissements d'enseignement et de recherche français ou étrangers, des laboratoires publics ou privés. 
Assumpció TOLEDO I MUR, ANNE LAGARRIGUE

Les ensembles céramiques du Bronze ancien et du Bronze final IIIa d'El Camp del Viver

(Baho, Pyrénées-Orientales)

- Fabrice PONS, Sébastien PANCIN, Roberta BEVILACQUA, Hélène MARTIN

Un habitat de la fin du premier âge du Fer dans le Toulousain: le site de Sauzas à Blagnac (Haute-Garonne)

- IAN ARMIT, TIM HORSLEY, FRÉDÉRIC MARTY.

Le Castellan (Istres, Bouches-du-Rhône) : résultats de prospection géophysique

- AnNie MONTÉCINOS, RÉJAne ROURE, SÉBAstien MUNOS

L’agglomération protohistorique de Mèze (Hérault): nouvelles données sur l'âge du Fer

- Alexandre BeYlier, Anne-Marie CURÉ, Cécile DUbOSSE, Éric GAILlEDRAT . avec la collaboration de SÉBASTIEN MUNOS et de SÉvERINE SANZ-LALIBERTÉ

Le dépôt de la «fouille 47» du Cayla de Mailhac (Aude): un ensemble du V $\mathrm{V}^{\mathrm{e}}$. av. n. è. à caractère rituel?

- Axel CAUVIN

La partie méridionale du premier village du quartier de l'Île à Martigues (Bouches-du-Rhône) entre le milieu du $\mathrm{V}^{\mathrm{e}}$ s. et la fin du $\mathrm{III}^{\mathrm{e}}$ s. av. J.-C.

- LoḮ BUFFAT, Bernard DEDET.

avec la collaboration de CRISTINA GARCIA DALMAU et AlEXANDRE AYASSE

L'ensemble funéraire du second âge du Fer de la Grange Blanche à Courthézon (Vaucluse)

- Patrice Courtaud, Elisabeth ROUSSEAU, Henri DUdAy, Philippe BOISSinOt

Les restes humains de l'âge du Fer de Roquepertuse (13) : fouilles anciennes et récentes 



\section{La partie méridionale du premier village du quartier de l'île à Martigues (Bouches-du-Rhône) entre le milieu du $V^{e}$ s. et la fin du III s. av. J.-C.}

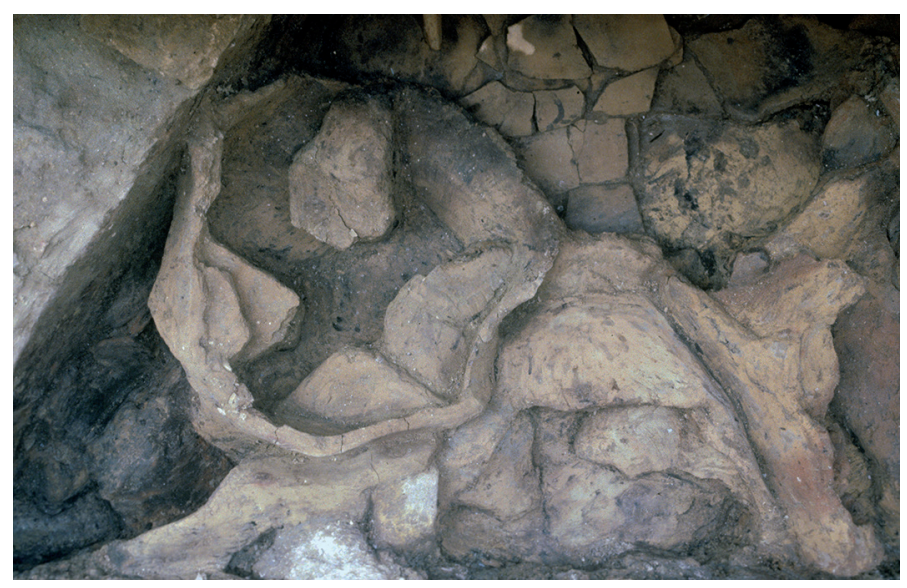

Résumé:

Cet article présente les résultats d'une campagne de fouille réalisée en 1989 dans le quartier de l'île à Martigues, mettant au jour de nombreuses structures du second âge du Fer. Ces découvertes, appartenant principalement au premier village, ont permis de confirmer les limites méridionales de l'agglomération protohistorique occupée entre le milieu du $V^{e}$ s. et l'extrême fin du III ${ }^{e}$ s. av. J.-C. La reprise de la documentation de fouille a également conduit à la mise en exergue d'un plan organisationnel différent lors des premières périodes d'occupations, qui était supposé lors de la fouille. Enfin, les séquences stratigraphiques bien connues dans cet habitat permettent de présenter un mobilier assez riche et surtout bien daté s’insérant dans un faciès céramique régional cohérent représentatif des sites proches de Marseille.

Mot-clé:

Provence occidentale, second âge du Fer, agglomération protohistorique, Martigues, céramiques.

\section{Abstract:}

This article presents the results of an excavation campaign carried out in 1989 in the district of L'Île in Martigues, revealing many structures of the second Iron Age. These discoveries, mainly belonging to the first village, confirmed the southern limits of the protohistoric agglomeration occupied between the middle of the $\mathrm{V}^{\text {th }}$ century and the end of the $\mathrm{III}^{\text {th }}$ century BC. The resumption of the excavation documentation also led to the highlighting of a different organizational plan during the first periods of occupation that was already supposed during the excavation. Finally, the stratigraphic sequences well known in this habitat make it possible to present a rather rich and especially well-dated furniture fitting into a coherent regional ceramic facies representative of the sites near Marseille.

Keywords:

West Provence, second Iron Age, Protohistoric agglomeration, Martigues, ceramics 


\section{Introduction}

Le quartier de l'île à Martigues ${ }^{1}$ occupe une position centrale au sein de cette ville des Bouches-du-Rhône, située sur les rives de l'étang de Berre, à 25 kilomètres à l'ouest de Marseille. En plein centre-ville de l'agglomération actuelle, il se situe précisément au débouché du chenal de Caronte, qui relie l'étang au golfe de Fos et à la Méditerranée.

Avec d'autres sites archéologiques du pourtour de l'étang, il marque l'importance qu'avaient revêtue, depuis la fin de la Préhistoire récente, l'occupation et l'exploitation humaine du territoire dans cette partie occidentale de la basse Provence. Pour l'âge du Fer, son emplacement, au carrefour de voies terrestres et maritimes, lui confère une place privilégiée dans les échanges commerciaux, l'étang de Berre étant durant toute cette période une région particulièrement dynamique (Chausserie-Laprée 2005, 42). Si le site même de l'Île ne paraît pas occupé avant le milieu du $\mathrm{V}^{\mathrm{e}} \mathrm{s}$. av. J.-C., on doit signaler que les rives toutes proches du chenal de Caronte sont le siège de plusieurs habitats antérieurs en particulier à la fin de l'âge du Bronze (L'Abion et les Salins de Ferrières) et dans la première moitié du VI' s. av. J.-C. (site de l'Annonciade) (Legros 1986; Chausserie-Laprée 2005; Marino, Damotte, Mercurin 2009, 148) (fig. 1).

La connaissance que nous avons aujourd'hui du site protohistorique du quartier de l'île est le fruit de plusieurs décennies de recherches. Si elles ont débuté en 1977 à l'oc-

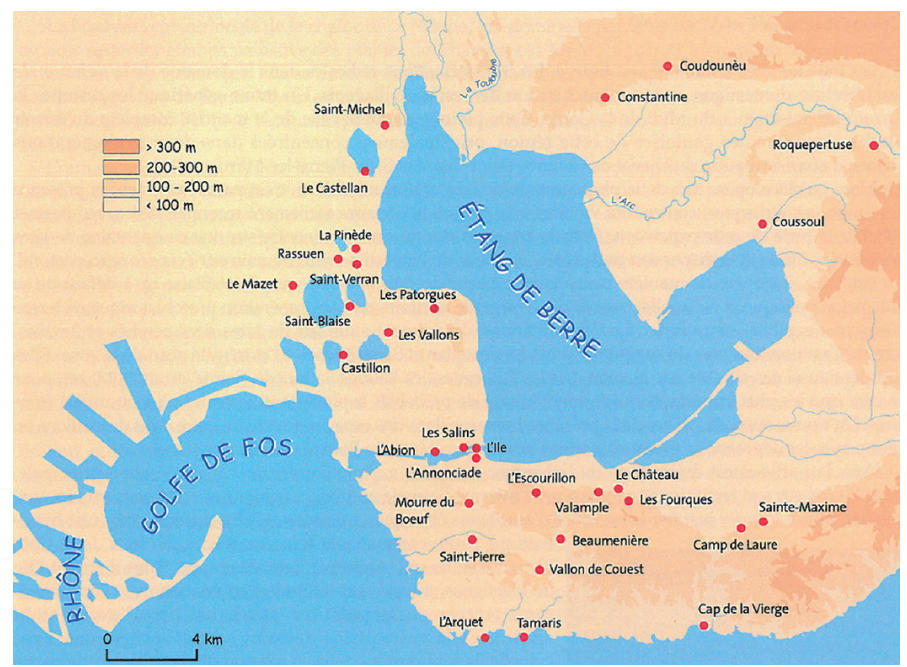

1 Carte de la région de l'étang de Berre du Vle au Ve $s$. Localisation des principaux sites d'habitats (d'après Chausserie-Laprée 2005, 46). casion d'une opération de restructuration du centre-ville moderne, ce n'est qu'à partir de 1982, que les archéologues ont pu réaliser des fouilles de qualité. Durant huit années (1982-1989), elles ont pris la forme d'opérations archéologiques de sauvetage en aire ouverte, qui précédaient les chantiers de construction. Fournissant des données scientifiques remarquables, elles ont permis d'identifier et d'explorer dans le sous-sol de ce quartier, les structures d'habitat et de défense d'un des sites gaulois les mieux conservés du Midi de la France. Après une longue interruption, il a fallu attendre 2001, puis 2003, pour que deux nouvelles fouilles d'urgence viennent compléter notre documentation, nous permettant de mieux saisir l'évolution et la chronologie de cet habitat (Chausserie-Laprée 2005, 17-24) (fig. 2, A).

$\mathrm{Au}$ terme de ces différentes fouilles, l'occupation protohistorique du quartier de l'Île peut être divisée en deux grandes étapes, marquées par la superposition et la succession de deux structures villageoises très différentes:

- un premier village occupé entre le milieu du $\mathrm{V}^{\mathrm{e}} \mathrm{s}$. av. J.-C. jusqu'à sa destruction définitive à la fin du $\mathrm{III}^{\mathrm{e}} \mathrm{s}$. av. J.-C.

- un second village, fondé au début du $\mathrm{II}^{\mathrm{e}} \mathrm{s}$. av. J.-C., et détruit à la fin de ce même siècle

\section{La campagne de fouilles de 1989}

D’une durée de cinq mois, entre mars et juillet 1989, cette campagne de fouilles est venue clôturer le long cycle d'opérations archéologiques de sauvetage, menées presque en continu dans le quartier de l'Île depuis la découverte du site en 1977. Succédant à des campagnes de fouilles extensives, qui avaient permis de mettre au jour et d'identifier les structures d'habitat appartenant aux deux villages gaulois successifs qui occupent le site, cette opération a pris une forme toute différente. Elle était motivée par la programmation de travaux de restauration et d'assainissement (par la pose de drains) de l'église de la Madeleine, classée Monument Historique, et a consisté dans la réalisation de tranchées de sondages, préalables aux travaux, à l'intérieur comme à l'extérieur de l'édifice.

Cette campagne de 1989 était restée en très grande partie inédite, en particulier en ce qui concerne le mobilier archéologique et le détail des vestiges mis au jour. Son importance s'est pourtant d'emblée révélée très grande, car c'est grâce à elle qu'a pu être restitué dans sa globalité le plan du premier village gaulois de l'île. 


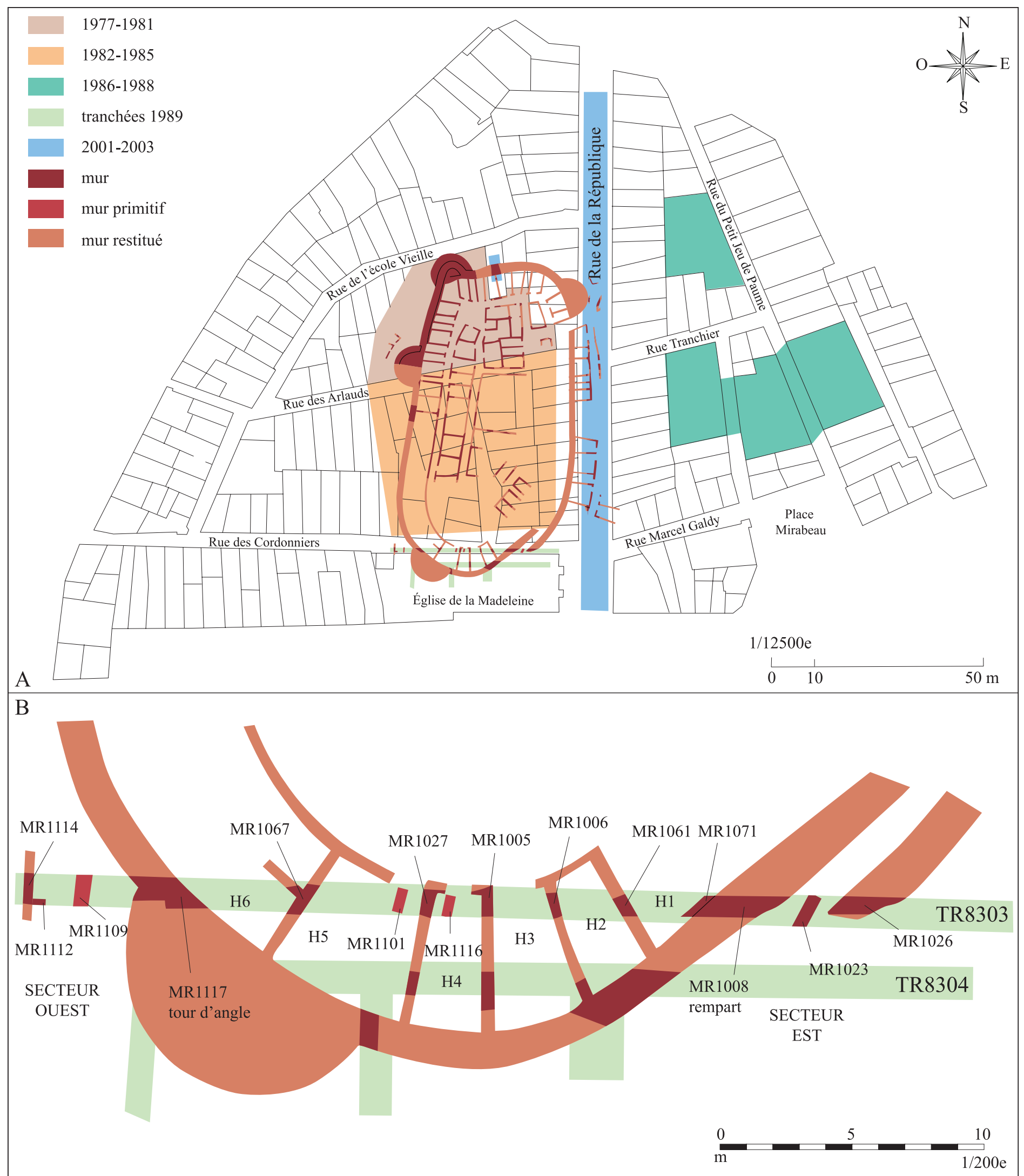

2 A Plan des différentes campagnes de fouille réalisées dans le quartier de l'île avec la restitution du plan du premier village protohistorique (d'après Chausserie-Laprée 2005, 17 et 96).

I B - Détail de l'îlot $H$ et de ses différents secteurs (A. Cauvin). 
C'est en effet l'unique campagne de fouille qui nous renseigne sur la partie méridionale de cette petite agglomération, nous en donnant en particulier les limites à travers le positionnement de son mur d'enceinte et d'un îlot d'habitations adossées (fig. 2, B).

\subsection{Les différents sondages et leurs principaux résultats}

Au sein de l'église, formée d'une grande nef unique, les investigations ont d'abord concerné, au pied des petits autels adossés contre son mur nord, une tranchée de $30 \mathrm{~m}$ de long sur $0,80 \mathrm{~m}$ à $1 \mathrm{~m}$ de large. Se rattachant perpendiculairement à cette tranchée, deux petits sondages complémentaires ont permis d'explorer la partie centrale de la nef. Ces trois excavations constituent le sondage 8904. Derrière le maître-autel situé dans le chœur, une dernière tranchée d'axe nord-sud, de $10 \mathrm{~m}$ de long et $1 \mathrm{~m}$ de large, correspond au sondage 8905 .

Cet ensemble de tranchées et sondages à l'intérieur de l'église a livré des vestiges de nature très variée, souvent difficiles à mettre en relation entre eux et à recaler par rapport aux structures protohistoriques déjà connues. La raison principale de cette difficulté tient non seulement à l'exiguïté des tranchées qui rendait difficile la lecture des strates et constructions mises au jour, mais surtout à l'importance des perturbations occasionnées dans le sous-sol par l'utilisation de l'édifice religieux comme lieu de sépulture durant la période moderne. Outre un grand nombre d'ossements humains, souvent sans connexion et accompagnés de chaux, qui parsèment les terres sous-jacentes au dallage en pierre de la nef, l'élément le plus notable est un caveau en pierre de taille de La Couronne, qui porte sur l'une des dalles supérieures l'inscription S CLER, désignant l'un des personnages inhumés dans ce tombeau. Il pourrait s'agir d'un homme enterré en octobre 1653 si l'on en croit l'inscription tronquée, mais déchiffrable sur le pourtour des dalles conservées (fig. 3, A et B).

Pour la période protohistorique qui nous intéresse ici, les vestiges les plus remarquables et facilement identifiables sont les deux tronçons de la fortification du premier village gaulois qui marque ici la limite sud de l'habitat. On les a repérés dans le sondage 8904, aussi bien dans la tranchée principale d'axe est-ouest que dans les deux sondages perpendiculaires, en particulier au contact du caveau (fig. 4). Montrant une nette inflexion vers l'ouest, la muraille est faite ici d'un mur simple d'environ 1,50 m de large, composé de blocs et moellons liés à l'argile, qui paraît s'enfoncer profondément dans le sous-sol.
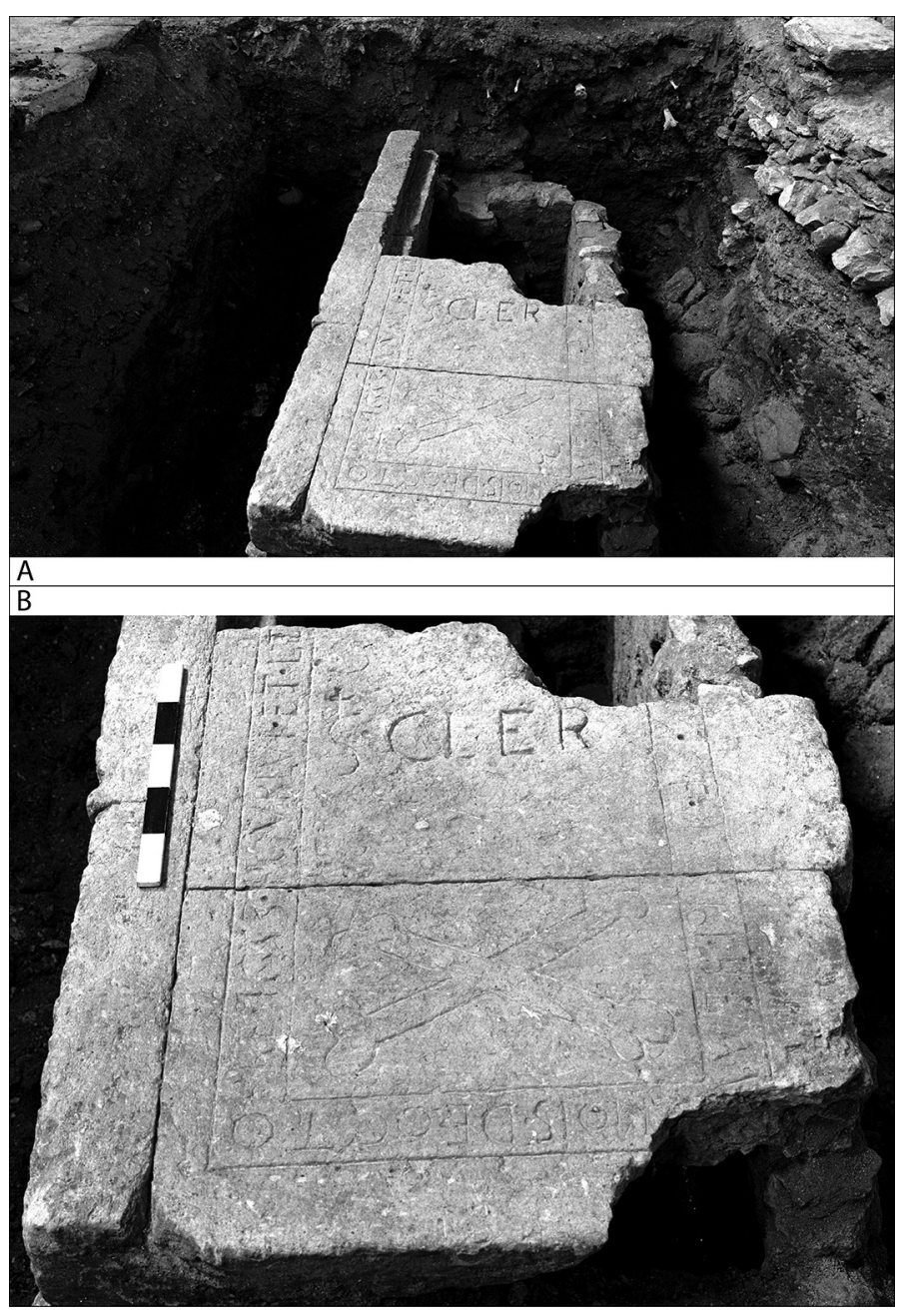

I 3 Caveau en pierre de taille de La Couronne mis au jour dans l'église de la Madeleine (photos J. Chausserie-Laprée).

I A Caveau dans son contexte lors de la fouille.

I B Photo de détail de l'inscription «S CLER» sur la dalle du caveau.

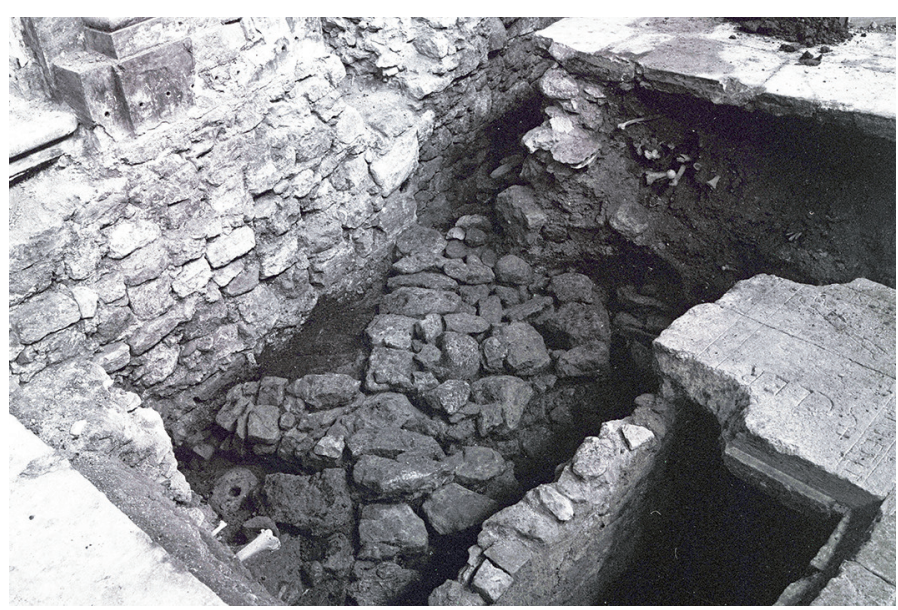

- 4 Tronçon du rempart dans l'église de la Madeleine dans la tranchée 8904 avec, sur la gauche, le départ du mur entre la maison $\mathrm{H} 2$ et H3 (photo : J. Chausserie-Laprée). 
Les structures d'habitat gaulois mises au jour dans ces tranchées intérieures à l'église sont à la fois modestes et d'une interprétation difficile. À l'ouest et au nord du rempart, quelques murs de pierres liés à la terre appartiennent à l'îlot simple d'habitations (îlot $\mathrm{H}$ ) adossé à la fortification du premier village gaulois, que l'on appréhende bien mieux au sein de la tranchée extérieure (8903). À l'est, plusieurs maçonneries, adossées ou non à l'enceinte, appartiennent à des constructions, parfaitement en place, que l'on doit probablement rattacher aux extensions successives qui marquent l'habitat, d'abord au milieu du IV ${ }^{\mathrm{e}}$ s. av. J.-C., puis au II ${ }^{\mathrm{e}}$ S. av. J.-C. avec l'occupation du second village gaulois (fig. 5). Le mur mis au jour au sein du sondage 8905, assez loin à l'ouest de la fortification, relève du même principe.

Le mobilier archéologique issu de ces tranchées 8904 et 8905 est à la fois trop peu abondant et trop mal calé pour être intégré dans cette étude ${ }^{3}$. Celle-ci ne concerne en effet pratiquement que les vestiges et objets archéologiques principalement des céramiques - issus de la fouille du sondage 8903, effectué à l'extérieur de l'église et au pied de son mur nord, dans la rue des Cordonniers.

Il s'agit d'une tranchée de $40 \mathrm{~m}$ de long pour environ $1 \mathrm{~m}$ de large. Bien souvent, l'espace exploré est plus étroit encore, à cause des fondations de l'église dont le ressaut réduit la largeur de la fouille à $80 \mathrm{~cm}$. Malgré le caractère étriqué et contraint de cette exploration en tranchée, l'excellente conservation des vestiges bâtis et la bonne lisibilité des strates protohistoriques correspondantes nous ont non seulement permis de comprendre l'organisation des diverses constructions mises au jour au sein de ce sondage 8903, mais nous ont fourni aussi, le plus souvent, les repères stratigraphiques et chronologiques pour l'interprétation et la datation de ces vestiges.

Le principal acquis de cette longue coupe transversale d'axe est-ouest concerne l'approche détaillée de la partie méridionale du premier village gaulois de l'Île. Hormis quelques couches et constructions mises au jour dans les niveaux supérieurs, qui appartiennent soit au second village gaulois soit à des ensembles plus tardifs remaniés, l'essentiel de la documentation retirée de cette tranchée doit être rattaché à l'habitat protohistorique initial. Elle concerne plus précisément six habitations de l'îlot $\mathrm{H}$, qui s'appuient contre la fortification sud de l'agglomération et dont l'occupation s'étend de la seconde moitié du V $V^{\mathrm{e}} \mathrm{s}$. av. J.-C. à la fin du III ${ }^{\mathrm{e}}$ s. av. J.-C. Aux deux extrémités de la tranchée, ont été mises au jour les maçonneries de la muraille ainsi que des strates et structures extérieures attenantes. Nous y reviendrons plus loin en détail.

\subsection{Données anciennes, intérêt et limites de l'étude}

Si la fouille en tant que telle est restée très largement inédite, nombreuses sont les données de ces sondages à avoir été intégrées dans des publications sur tel ou tel aspect du site gaulois de l'Île de Martigues. On peut distinguer d'une part les études et synthèses concernant les structures bâties et les aménagements domestiques (Chausserie-Laprée, Nin 1990 et plus largement Chausserie-Laprée 2005), d'autre part celles qui traitent d'éléments du mobilier ar-

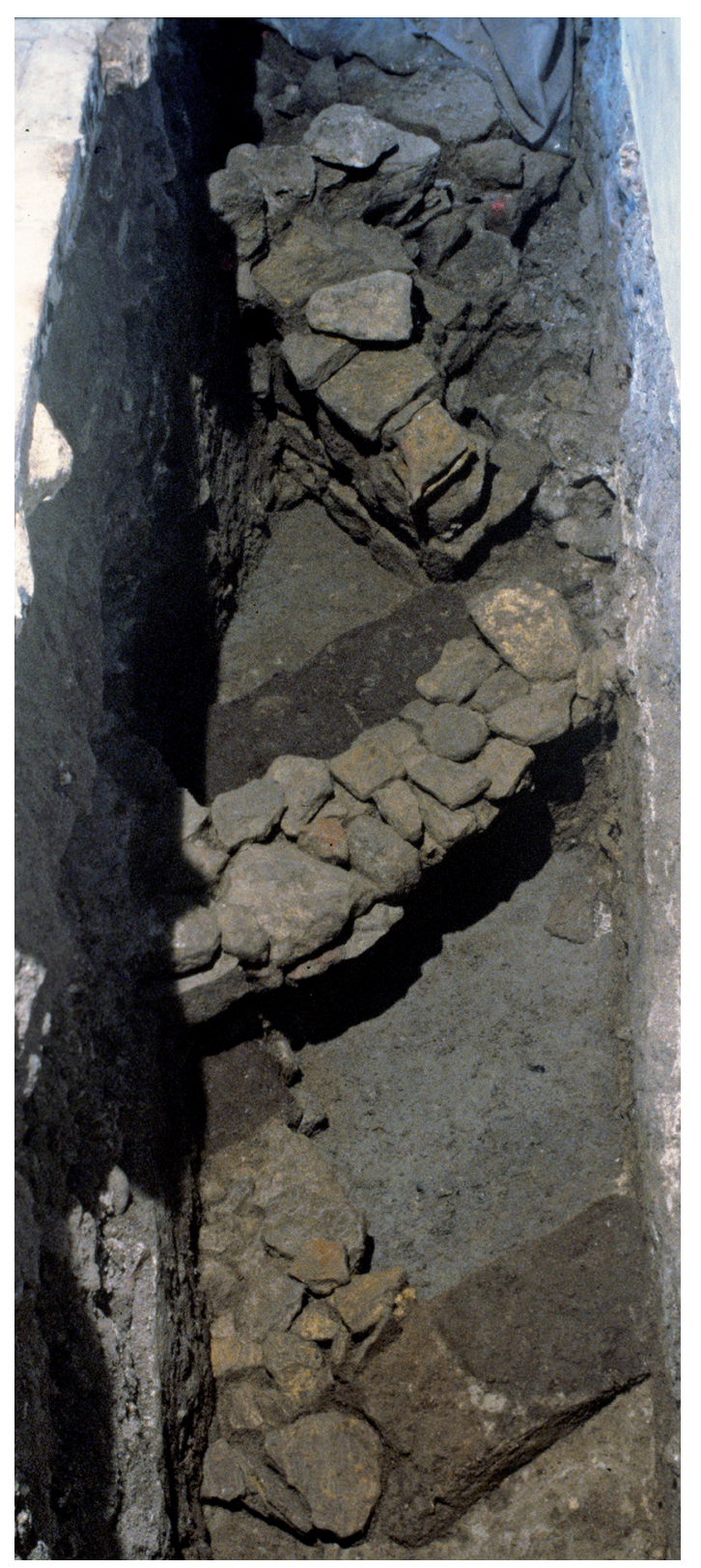

I 5 Construction en pierre et en terre crue hors des murs de l'agglomération dans la tranchée 8904 à l'intérieur de l'église de la Madeleine - vue de l'est (photo : J. Chausserie-Laprée). 
chéologique. Parmi ces dernières, outre les publications déjà mentionnées sur la céramique grise monochrome de l'Île (Chausserie-Laprée, Nin 1995) et les amphores des $\mathrm{VI}^{\mathrm{e}}-\mathrm{IV}^{\mathrm{e}}$ siècles av. J.-C. (Chausserie-Laprée 2004), on doit citer le travail de master d'Antoine Ratsimba (2005) sur les pithoi de Gaule méridionale, l'étude concernant les meules des différents habitats martégaux (Chausserie-Laprée 1998), enfin le mobilier métallique du premier village gaulois de l'île (Rétif 2000). Mais le mobilier issu du sondage 8903 n'a jamais été étudié en tant que tel et dans son ensemble comme cela a pu être fait pour plusieurs habitations de la partie nord du village (Damotte 2003). Le principe de cet article est donc de présenter de manière détaillée la quasi-totalité d'une collection archéologique qui a pu pour l'essentiel être précisément recalée aussi bien en plan qu'en stratigraphie.

Partis des données brutes de terrain (plans, schémas, photos, fiches d'US), nous avons dû surmonter l'écueil que représentait le contexte particulier de la fouille au sein d'une tranchée très étroite. Absence de vue d'ensemble, lisibilité parfois difficile des couches au sein d'espaces très réduits, caractère tronqué des vestiges mis au jour voire du matériel recueilli, sont autant de difficultés que nous avons pu dépasser, au moins pour ce qui concerne l'approche chronologique et quantitative du mobilier. Parmi les réserves que l'on doit formuler sur cette approche, et malgré la relative abondance de la collection - elle représente plusieurs milliers de fragments -, il faut mentionner d'abord le caractère disparate de cet ensemble qui privilégie nettement la période 2 au détriment des autres périodes d'occupation. Plus encore que dans d'autres secteurs du site, on le doit d'une part à la difficulté d'atteindre, au sein d'une tranchée si étroite, les niveaux d'occupation les plus anciens (période 1) le plus souvent inaccessibles car noyés sous le niveau de la nappe phréatique, d'autre part à la mauvaise conservation ou la pauvreté des niveaux les plus récents (période 4 surtout). Ceux-ci sont en effet le plus souvent recoupés par les quelques vestiges du second village gaulois ou de la période moderne qui apparaissent dans les couches supérieures, immédiatement sous les remblais d'époque contemporaine.

Par ailleurs, les circonstances de la fouille en tranchée de cette partie sud du village, qui restitue une image trop tronquée et partielle des espaces domestiques, nous a empêché de tenter ici une approche spatiale du mobilier, débouchant sur une étude quasi ethnographique des maisons gauloises, qui avait été réalisée pour les habitations du quartier nord-ouest du village (Damotte 2003).
Malgré ces réserves, l'homogénéité et la qualité documentaire des structures et mobiliers mis au jour au sein de la tranchée 8903 , très conforme à la documentation déjà étudiée, nous ont permis de nous appuyer sur la très longue expérience acquise lors des fouilles extensives précédentes. Elles ont en particulier rendu possible la distinction précise des différentes strates et périodes d'occupation, qui voient alterner niveaux d'habitat et couches de destruction ou d'abandon. Parmi ces dernières la plus notable demeure l'épisode violent qui marque la destruction par incendie de toutes les maisons du village un peu avant le milieu du IV $\mathrm{s}$. av. J.-C. Son identification systématique s'est avérée ici un marqueur stratigraphique et chronologique décisif pour l'étude de la collection.

\section{Cadre de l'étude}

Le travail présenté ici ${ }^{4}$ s'intéresse à la stratigraphie et à l'étude du mobilier (vaisselle et matériel de transport) issu du sondage $8903^{5}$ qui ont touché la structure de l'îlot $\mathrm{H}$ dans la partie sud du premier village de l'Île. La période chronologique concernée couvre toute l'occupation de cet habitat, du milieu du $\mathrm{V}^{\mathrm{e}} \mathrm{s}$. av. J.-C. jusqu'à la fin du $\mathrm{III}^{\mathrm{e}} \mathrm{s}$. av. J.-C. L'un des objectifs de l'étude de ce matériel est donc de s'intéresser aux évolutions quantitatives sur le temps long pour identifier de possibles tendances.

\subsection{Chronologie}

La présentation des phases d'occupation et de leur datation reprend celle des anciennes publications qui ont concerné les données issues de la partie nord-ouest du village (Chausserie-Laprée, Nin 1990, 1995 ; Damotte 2003).

- La période 1 correspond à la fondation du village et à l'installation ex nihilo des premiers habitants sur le site. Datée entre 450 et 425 av. J.-C., elle s'achève par une couche de destruction à la fin du $\mathrm{V}^{\mathrm{e}} \mathrm{s}$. av. J.-C. Son mobilier céramique se compose essentiellement de céramique non tournée.

- La période 2 est quant à elle divisée en trois temps : o La période 2a est celle qui correspond à la réoccupation immédiate du site et à la reconstruction de l'habitat après ce premier incendie. Très proche de la période précédente, et s'étendant de 425 400 av. J.-C., elle se traduit par d'importantes modifications architecturales au sein des îlots. 
- La période $2 \mathrm{~b}$, dans la continuité de la période précédente, se caractérise notamment, au sein du mobilier céramique, par un accroissement de la vaisselle tournée et des productions non tournées des rives de l'étang de Berre. Dans le sud du village, c'est durant cette période que l'îlot $\mathrm{H}$ paraît se mettre en place et se structurer. Elle est datée du premier quart du IV $\mathrm{IV}^{\mathrm{s}}$ s., soit environ de 400 à 375 av. J.-C.

- La période $2 \mathrm{c}$ correspond à la deuxième couche d'incendie attestée sur l'ensemble de l'habitat. Résultat d'une attaque militaire du village, elle est placée à la charnière du premier et du deuxième quart du IVe av. J.-C., entre 375 et 360 av. J.-C.

- La période 3 est divisée en deux temps :

$\circ \mathrm{La}$ période $3 \mathrm{a}$ correspond à la reconstruction immédiate du site après l'importante destruction précédente. Elle se caractérise par un retour à un répertoire céramique plus traditionnel, avec de nouveau une part plus importante de la céramique non tournée. Perçue comme un moment de récession économique, elle ne dépasse pas le dernier quart du IV s. av. J.-C.

$\circ$ La période $3 b$, beaucoup plus longue, qui voit l'apparition des productions italiques, est datée entre la fin du IV e s. av. J.-C. et la fin du III' ${ }^{\mathrm{e}}$ s. av. J.-C., soit environ de 325 à 200 av. J.-C.

- La période 4 correspond à la dernière destruction du village qui survient vers 200 av. J.-C.

Chacune de ces périodes a été identifiée dans le sondage 8903. Malgré sa faible largeur, il a pu livrer de nombreuses élévations de murs d'enceinte et d'habitations, occupées sur la longue durée (fig. 6). Sur l'ensemble des sondages 8903 et 8904, six maisons ont donc été partiellement fouillées ainsi que des éléments du rempart du premier village à l'est et à l'ouest de ces maisons, formant en tout huit ensembles cohérents, au moins à partir de la période $2 \mathrm{~b}$. Pour les niveaux inférieurs, et en particulier lors de la période 1, la fondation des murs n'a pas toujours été atteinte en raison des remontées d'eau de la nappe. Quant aux niveaux supérieurs de l'habitat protohistorique, plusieurs fosses, tranchées de récupérations et murs modernes viennent perturber la stratigraphie, nous privant parfois d'une bonne approche des périodes ultimes de cette occupation.

\subsection{Durant les périodes 1 et $2 a$, une organisation primitive différente?}

Tels qu'ils sont présentés dans la dernière étude de synthèse sur l'habitat du premier village (Chausserie-Laprée, Nin 1990), le plan et la division de l'îlot $H$, adossé à la muraille, ne sont pas mis en place avant la période $2 b$, soit au tout début du $I V^{e} s$. Antérieurement, ils adoptent, en tout cas, une configuration différente, quoique difficile à mettre en évidence en raison du caractère tronqué et peu net des données recueillies. Sur la foi des constructions et

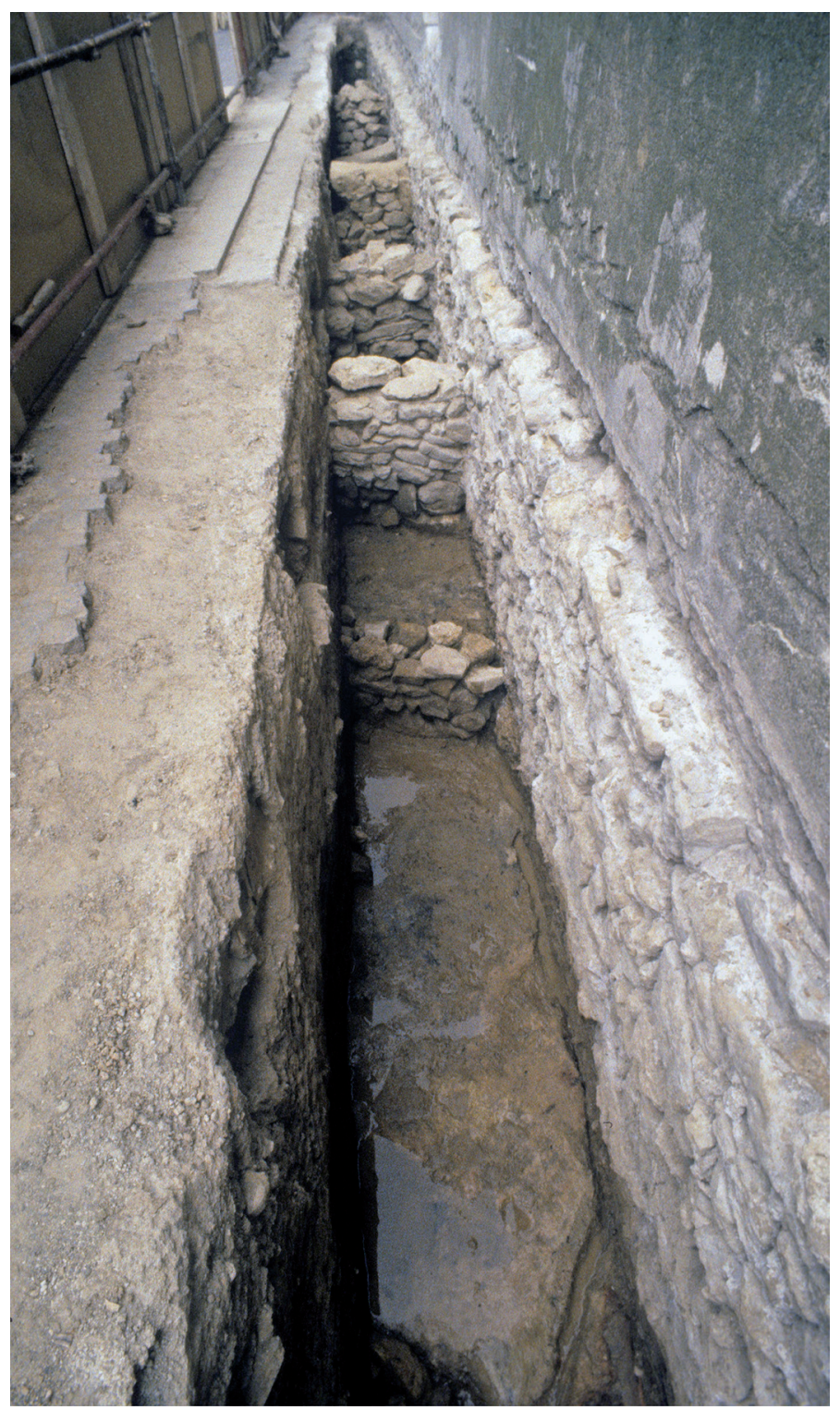

I 6 Vue générale de la tranchée 8903, dans la rue des Cordonniers, au nord du mur de l'église de la Madeleine, avec la succession des murs du premier au dernier plan : MR1101, MR1027, MR1005, MR1106.

Vue de l'ouest. (photo : J. Chausserie-Laprée). 
strates apparaissant à la base de la coupe, dans la partie ouest du sondage 8903 , on peut la qualifier ici de primitive. En effet l'alignement des murs les plus profonds n'est pas repris ensuite dans la division qui prévaut à partir du début du $\mathrm{IV}^{\mathrm{e}}$ s. av. J.-C. (fig. 7, Sections C, D et E). Associés à ces murs primitifs des périodes 1 et 2 a (les murs de maisons MR1116 et MR1101 ainsi que les éléments de rempart MR1117 et MR1109), plusieurs niveaux sont attestés dans la partie ouest de la tranchée 8903. Cette zone est très largement entamée par le creusement d'un large fossé (1085), qui a détruit toute la sédimentation gauloise et récupéré aussi jusqu’à leur fondation les éléments de la muraille principale. De la fortification, ne subsistent ici que des massifs parementés de blocs liés à l'argile, interprétés l'un comme un massif de renfort extérieur (MR1109), l'autre comme le départ d'une tour d'angle (MR1117). Ancrés dans une couche de sable presque vierge de mobilier (1111), ces différents éléments bâtis sont associés intra-muros à un niveau hétérogène et charbonneux (1115), qui correspond à la destruction initiale d'une habitation de grandes dimensions, appuyée au mur d'enceinte (période 1). Quant à l'espace plus modeste, délimité par les murs MR1116 et MR1101, les niveaux primitifs n'y ont pas été explorés.

Extra-muros, à l'ouest de MR1109, la sédimentation est caractérisée par une couche initiale noirâtre (1113), faite d'éléments organiques en décomposition (bois gorgés d'eau) qui n'a pu être fouillée entièrement à cause des remontées d'eau. La destruction de la période 1 est marquée ici par des amas de pierres (1110) interprétés comme l'effondrement de la muraille (fig. 8 et fig. 9).

Pour la période 2a, qui marque la reprise de l'occupation après cette première destruction, les données sont encore très lacunaires. Elles indiquent cependant une reconstruction à l'identique par rapport à la période précédente. Deux espaces habités sont délimités à l'abri de la muraille: pour la petite cellule à l'ouest, seul le sol cendreux 1104 peut y être sans doute associé; dans la grande maison appuyée à la muraille, la sédimentation étudiée est plus importante. Les niveaux 1106, 1107 sont des couches argilo-cendreuses, parsemées de débris de coquilles, avec des zones charbonneuses et rubéfiées, résurgence des couches d'incendie de la période 1.

Extra-muros, alors que le renfort MR1109, ruiné, n'est pas reconstruit, la période 2a est marquée par une couche dépotoir (1108) riche en mobilier archéologique, parmi lequel on note la présence d'une hache polie faite dans un galet de rivière (fig. 10, $\mathrm{n}^{\circ} 5$ ).

En définitive, malgré les circonstances de la fouille en tranchée, qui empêchent d'avoir une vision d'ensemble satisfaisante, l'exploration de cette zone méridionale du village gaulois de l'Île confirme les observations effectuées en 1984 au sein de l'îlot A, dans la partie nord-ouest du village. En effet, dans les deux cas, le découpage de l'habitat lors des premiers temps de son occupation n'est pas le même que celui qui prévaudra ensuite presque sans changements et que nous abordons ci-dessous.

\subsection{Durant les périodes $2 b$ à 4 , la structura- tion et l'occupation d'un îlot simple d'habita- tions adossées à la muraille (fig. 2, fig. 7)}

L'acquis principal des recherches menées dans les sondages 8903 et 8904 concerne la connaissance des constructions et de la sédimentation correspondante appartenant à l'occupation du premier village gaulois de l'île pour la phase qui s'étend entre la période $2 \mathrm{~b}$ et la période 4. Quoiqu'inégalement conservés selon les secteurs de la tranchée, les vestiges mis au jour permettent de définir la stratigraphie et la nature de l'occupation de six maisons gauloises, appartenant à un même îlot (îlot $\mathrm{H}$ ) formant l'extrémité sud du village. Plus précisément la tranchée 8903 marque une section longitudinale d'axe est-ouest à travers ces six habitations et les deux sections opposées de la fortification qui limite l'îlot $\mathrm{H}$ au sud-est comme au sud-ouest.

La description des structures et l'analyse du mobilier recueilli au sein de ces maisons forment l'essentiel de la matière de cet article. Nous appuyant principalement sur la coupe restituée que nous avons établie à partir des données du terrain (fig. 7), nous décrirons les différents espaces identifiés en procédant de l'est vers l'ouest.

\subsubsection{Le secteur est: une poterne à l'angle sud-est $d u$ rempart}

La partie est du sondage 8903 est occupée presqu'entièrement par les constructions d'un imposant dispositif maçonné, formé de plusieurs murs parallèles, traversant en oblique la tranchée (fig. 11). Lélément principal est un puissant mur de pierres liées à la terre (MR1008), qui marque la limite de l'habitat domestique. Son emprise initiale, large d'1,20 m, pourrait avoir été doublée à l'est par un mur de renfort (MR1008a) à plusieurs parements $\mathrm{d}^{\prime} 1,40 \mathrm{~m}$ de large ${ }^{6} .1$ mètre au-delà, vers l'est, on trouve un second mur (MR1026) de même nature et emprise, parallèle à MR1008. Au nord, ce massif MR1026 montre un parement fini, qui traduit ici très probablement l'existence d'un piédroit et donc d'un passage piéton. C'est sur cette base qu'a été émise l'hypothèse de l'existence, à ce point 


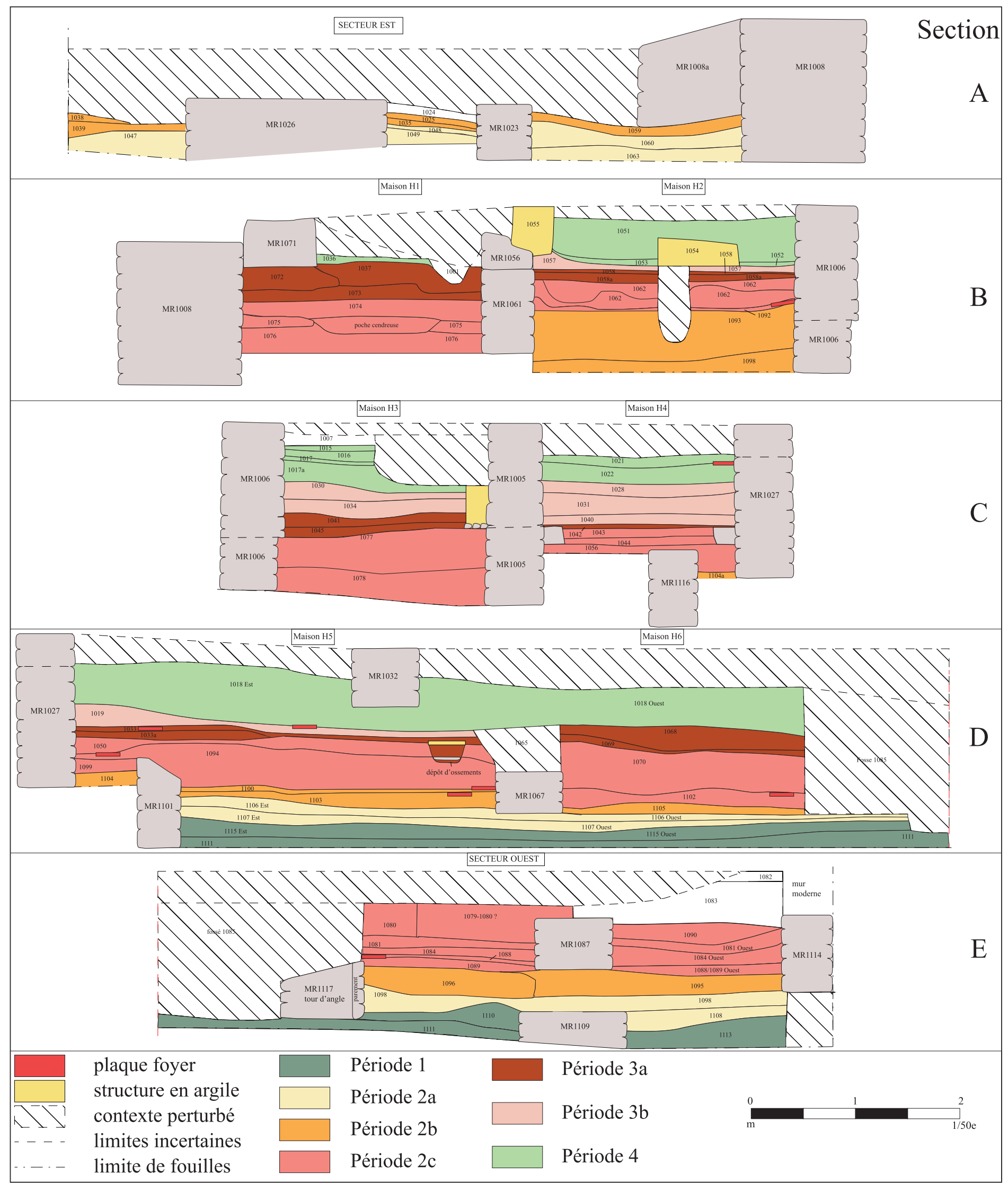

7 Restitution de la coupe stratigraphique de la tranchée 8903 d'après les altitudes prises sur les croquis et les plans lors de la fouille. Certains murs apparaissent très larges puisqu'ils sont obliques par rapport à la tranchée, notamment le rempart MR1008 ou encore MR1087 dans le secteur ouest. De nombreux sauts de coupes sont effectués, principalement à hauteur des murs, afin de restituer l'ensemble de la tranchée (A. Cauvin). 


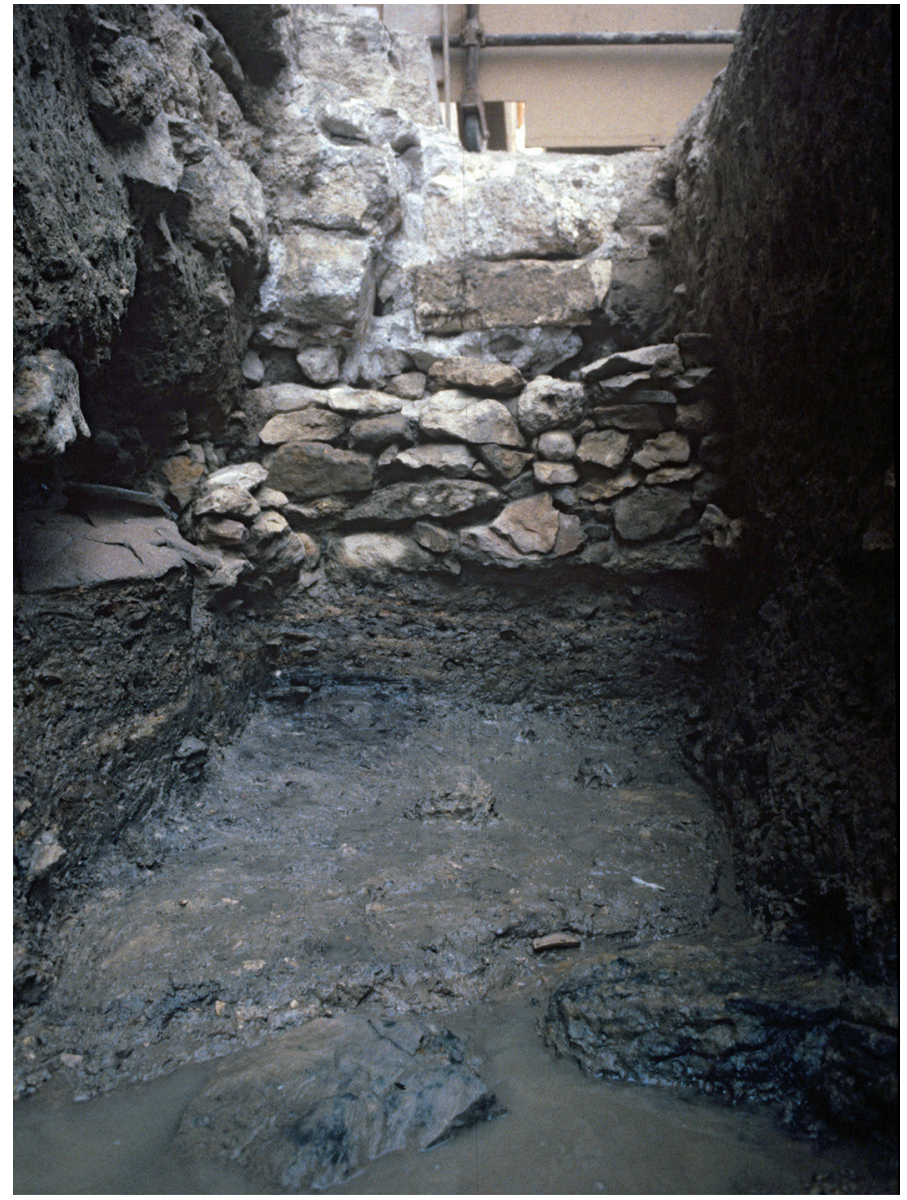

I 8 Fond de la tranchée 8903 après la fouille de l'US 1113 dans le secteur ouest, avec le mur protohistorique MR1114, surmonté et entamé par un mur moderne cimenté. Vue de l'est (photo J. Chausserie-Laprée).

précis du site, d'une poterne donnant accès au village. Cependant, aucun véritable niveau de circulation en place n'a été mis en évidence dans le couloir étroit de cette supposée porte à recouvrement. Les niveaux supérieurs (1024) (période 3 ou 4) apparaissent même remaniés?

Sous l'effondrement ou le doublage du mur MR1008, daté de la période 4, celle de la destruction du premier village, apparaissent quelques remblais homogènes $(1059,1060$ et 1063) qu'on peut dater grâce au matériel céramique, abondant et varié, de la période 2. Ces niveaux anciens sont curieusement associés à deux murs non perpendiculaires entre eux (MR1023 et MR1064), dont la présence dans cet espace dit de circulation, extérieur au rempart, interroge. La supposée poterne, peut-être en chicane, ne serait-elle qu'un aménagement tardif?

\subsubsection{La maison $\mathrm{HI}$}

Accolée au rempart MR1008, la maison H1 est recoupée sur environ 2,30 m jusqu'au mur MR1061, à l'ouest dans la

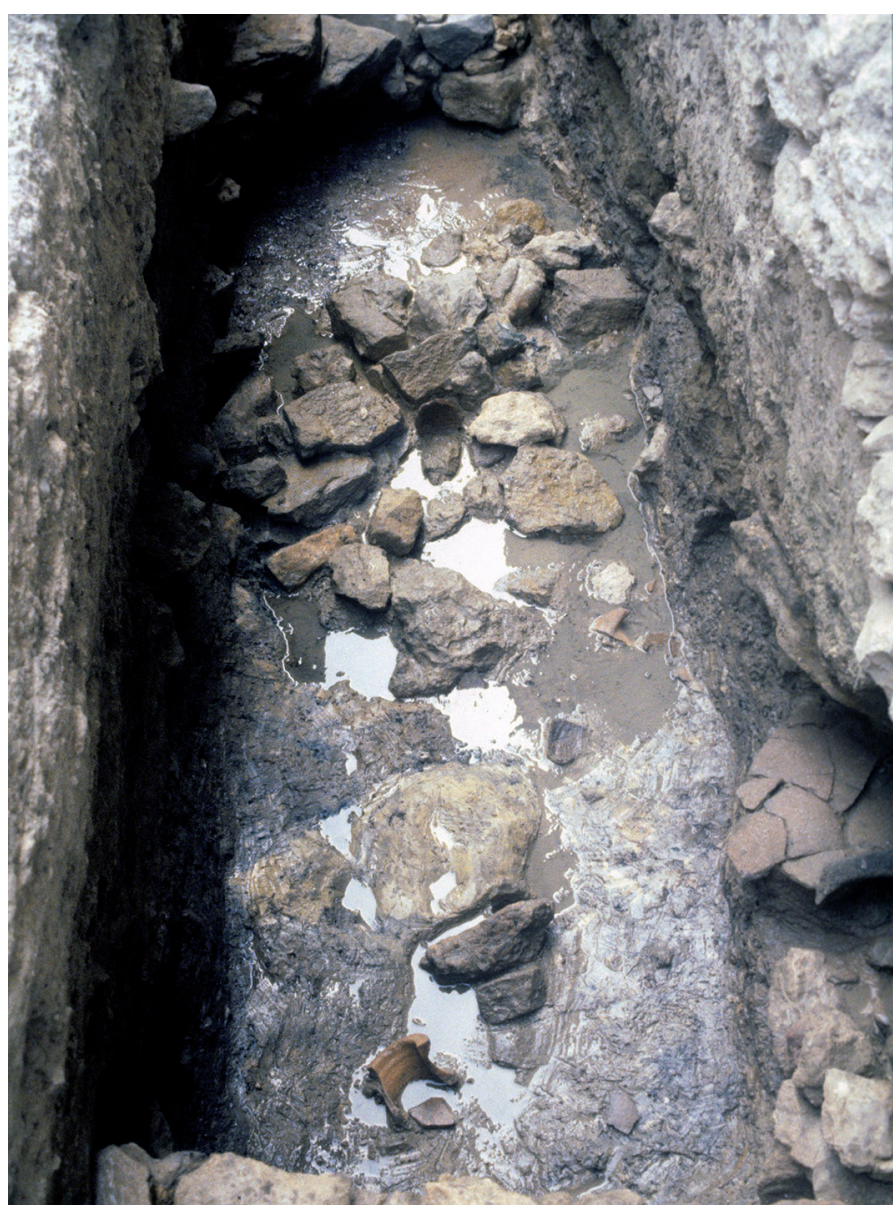

\section{9 Niveaux primitifs de la période 1 avec l'US 1108 et le mur MR1109 au centre. \\ Vue de l'ouest (photo J. Chausserie-Laprée).}

tranchée. Il s'agit d'un mur construit en briques crues de couleur grise sur un solin de pierres, constitué de trois assises de moellons calcaires liés à la terre. L'élévation en brique est très bien conservée, les joints d'argile jaune liant les sept assises de briques grises étant parfaitement visibles (fig. 12).

\section{Sédimentation, principaux aménagements et mobiliers (fig. 7, section B)}

Un seul niveau d'occupation est relatif à la période $2 \mathrm{c}$, la période d'incendie. Il s'agit d'un niveau d'effondrement de la toiture et des murs (1076), sur lesquels viennent se placer deux épaisses couches de terre crue fondue, riches en matériel céramique (1075 et 1074), en particulier de nombreux fragments d'une jatte en céramique non tournée, écrasée sur place contre le rempart (fig. 41, $\left.\mathrm{n}^{\circ} 1\right)^{8}$. Au sein de la couche 1074, on note aussi la présence d'un bracelet en bronze plié (Rétif 2000, 171, fig. 9, n 195). Au pied du rempart, toujours à l'intérieur de la maison, il faut noter la présence d'un dépôt d'ossements de faune dans une petite fosse. Malgré leur rareté sur le site de l'Île, c'est une pratique très bien attestée sur les 


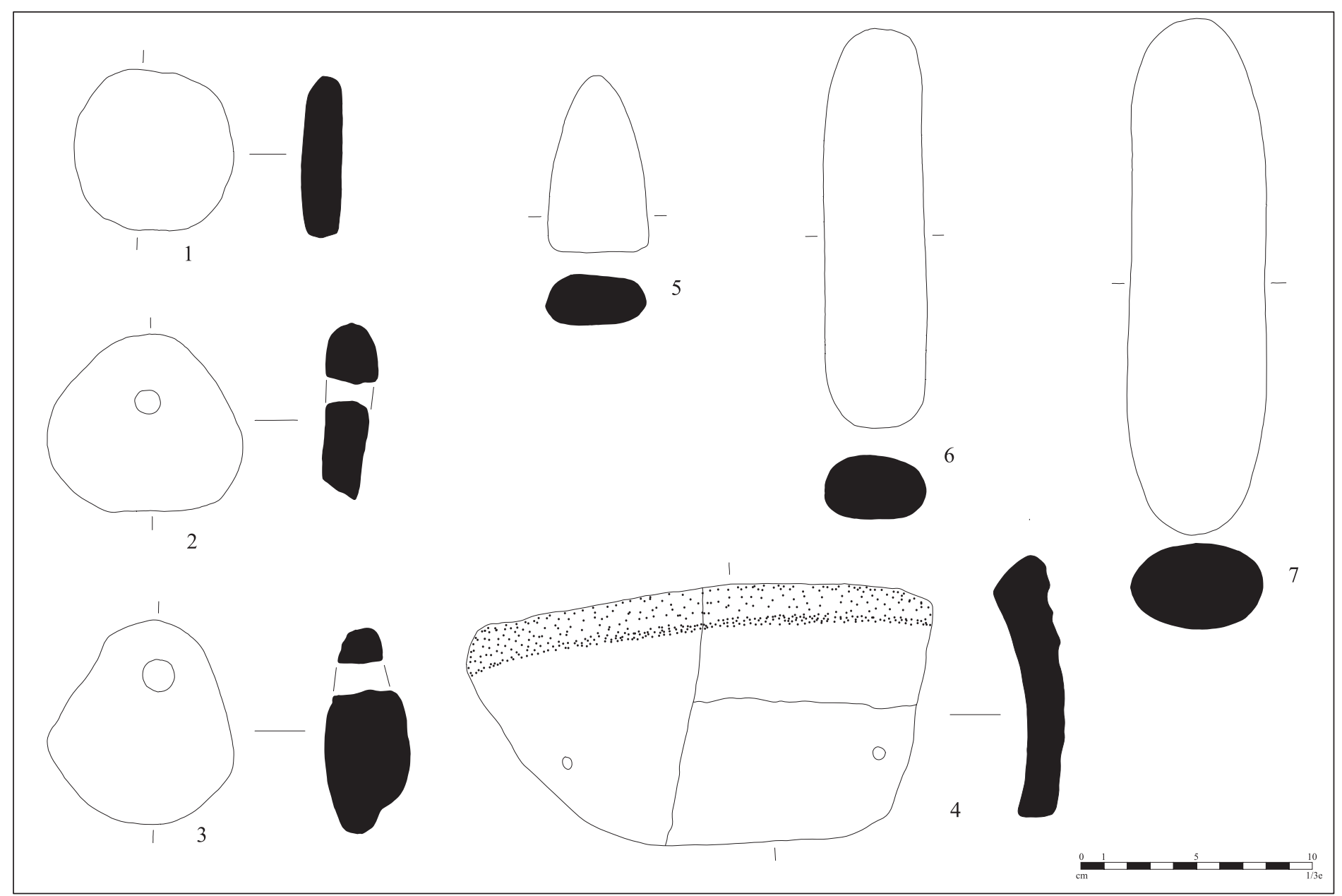

I 10 Petit mobilier - $n^{\circ} 1$ : jeton; $n^{\circ s} 2$ et $3:$ pesons en céramique ; $n^{\circ} 4$ : peson en mortier massaliète ; $n^{\circ} 5:$ hache polie ; $\mathrm{n}^{\text {os }} 6$ et 7 : pilon (ou concasseur en pierre) (dessin : Service Archéologie de la Ville de Martigues, D.A.O. : A. Cauvin).

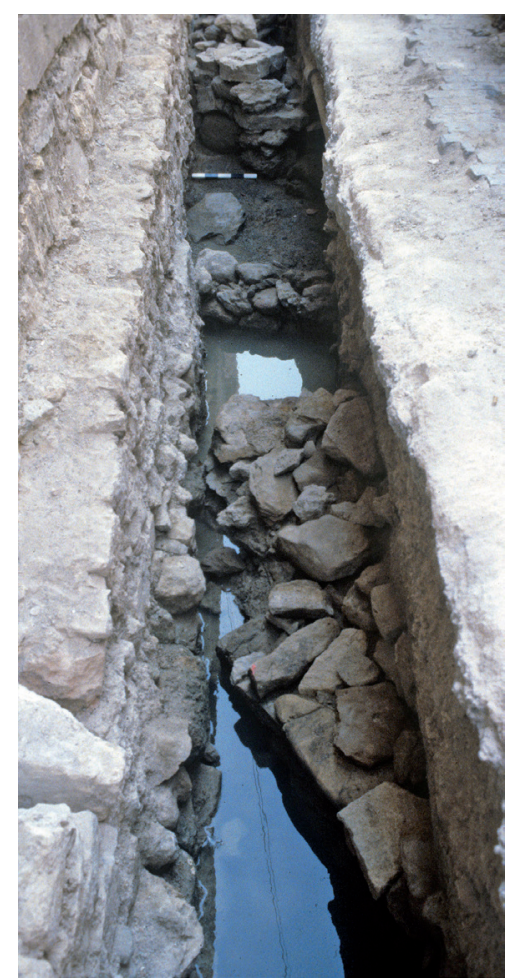

I1 Secteur est de la tranchée 8903 au premier plan MR1026, ensuite MR1023, puis au fond le rempart MR1008. Vue de l'est (photo :J. Chausserie-Laprée).

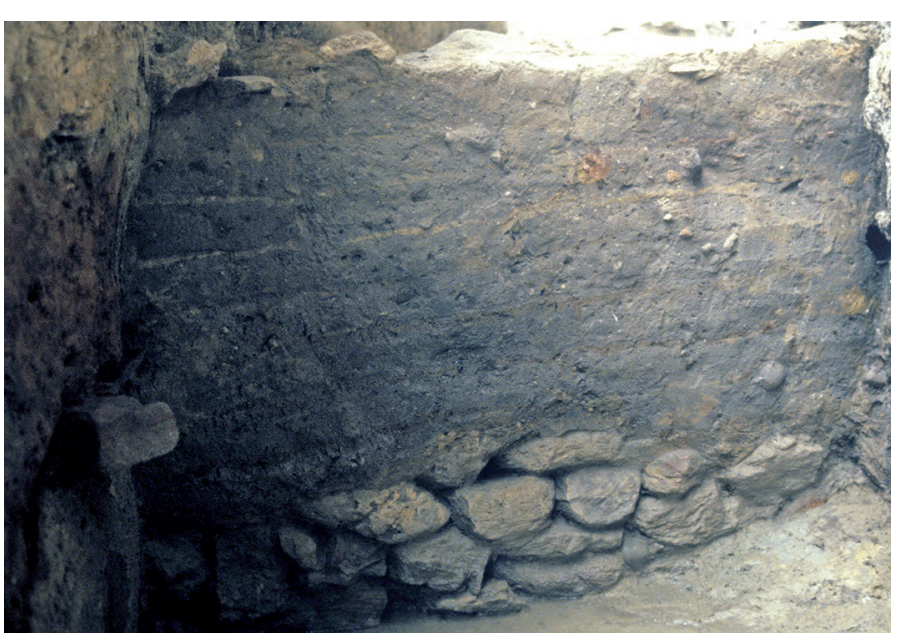

I 12 Mur MR1061 avec son élévation en briques crues et les joints sont encore visibles.

Vue de l'ouest (photo: J. Chausserie-Laprée). 
sites protohistoriques proches, tel l'oppidum de Saint-Pierreles-Martigues et le caractère symbolique de ce dépôt ne fait pas de doute (Nin 1999, 264-268; Chausserie-Laprée 2005, 232; Nin, Chausserie-Laprée 2016).

Pour la période 3, un niveau cendreux (1073) vient recouvrir ces dépôts: pauvre en matériel céramique, il comporte plusieurs plaques d'argile jaune-orangée et pierres plates et se trouve lui-même surmonté par un niveau d'effondrement de pierres (1072), inexpliqué lors de la fouille, mais provenant sans doute du rempart. Parmi ces pierres, des fragments d'une molette en andésite ont été découverts (ChausserieLaprée 1998, fig. 56, $\mathrm{n}^{\circ}$ 106).

L'ensemble est recouvert par un niveau d'effondrement (1036), composé de brique fondue et de fragments de vases en argile crue marquant la destruction du premier village

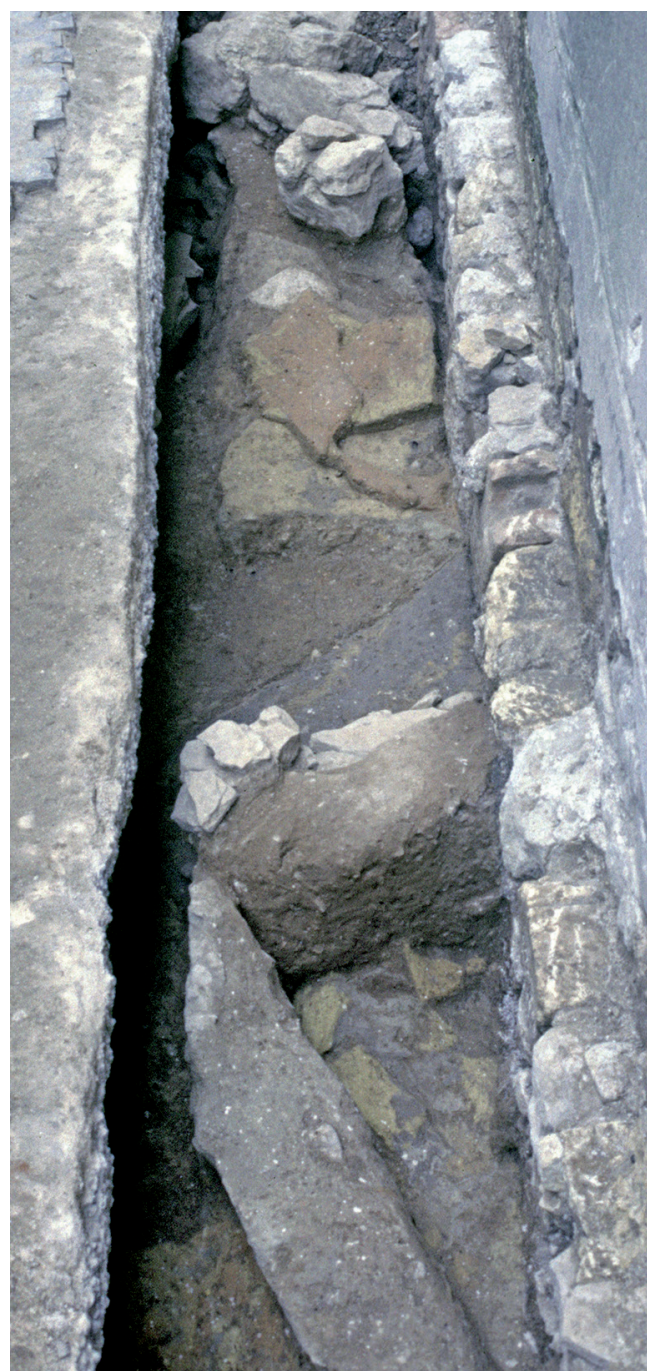

I 13 Au premier plan, les deux structures en argile 1055 et 1054 formant un angle. Au second plan, effondrement des superstructures en argile (1036) au pied du mur MR1071. Vue de l'ouest (photo: J. Chausserie-Laprée). daté de la période 4 , recouvrant une surface de sol légèrement cendreux (1037) (fig. 13). Ces niveaux sont associés au mur MR1071 venant se poser contre le rempart, pratique couramment attestée sur le site.

\subsubsection{La maison $\mathrm{H} 2$}

Mitoyenne de la maison H1 à l'est par le mur MR1061, décrit ci-dessus, la maison $\mathrm{H} 2$ est délimitée à l'ouest par le mur MR1006: elle occupe une emprise de 2,50 m de large. De cette maison, nous connaissons aussi son angle sudouest, mis au jour dans l'église (sondage 8904), où MR1006 vient s'appuyer contre la muraille (fig. 4). Dans la tranchée 8903, le mur MR1006 est constitué d'un soubassement de pierres liées à la terre argileuse, empilées sur neuf assises, et d'une élévation en brique crue. Cependant, la partie en pierres du mur n'est pas homogène, montrant une rupture dans l'appareil au niveau de la cinquième assise, qui laisse penser qu'il s'agit d'une superposition de deux murs (fig. 14). Cette pratique d'empilement des maçonneries sur les mêmes alignements, au fur et à mesure des reconstructions et réoccupations du site, a déjà été constatée dans le village de Martigues à de nombreuses reprises (ChausserieLaprée et al. 1984, 55).

\section{Sédimentation, principaux aménagements et mobiliers} (fig. 7 : section B)

Le niveau le plus ancien mis au jour (1098) dans cette maison remonte à la période $2 \mathrm{~b}$ et correspond à un sol d'habitat argilo-cendreux. Une épaisse couche de remblai jaune

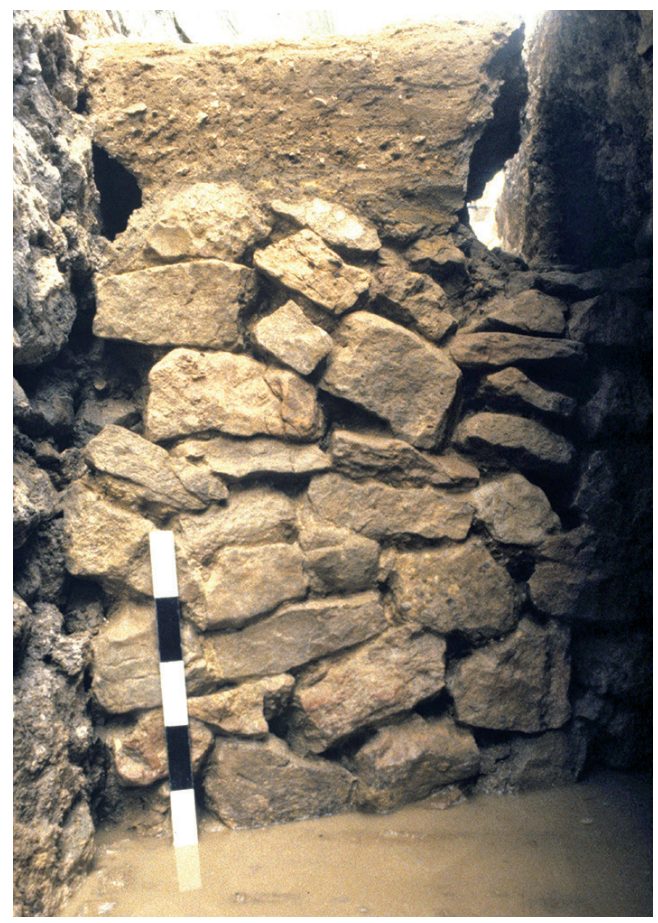

14 Empilement de murs successifs formant le mur MR1006. Vue de l'est (photo J. Chausserie-Laprée). 
sableux (1093), présentant quelques passes argileuses, vient le recouvrir. Elle appartient également à la même période. C'est au-dessus qu'a été dégagé le sol d'habitat d'argile grise (1092) (fig. 15), parfaitement caractérisé de la période 2c. Nous en détaillons ici les différents aménagements domestiques et architecturaux encore en place: l'angle nord-ouest de la maison est investi par une plaque foyer d'argile maçonnée dont n'apparaît que sa moitié sud. Elle est dépourvue de radier réfractaire. Associée à ce foyer, et extraite de la coupe nord de la tranchée, une grande meule dormante (type 6 b) très usée et cassée, en basalte vacuolaire, repose sur le sol tout à côté et en position de travail (ChausserieLaprée 1998, CDrom objet $n^{\circ}$ 87, fig. 53). À quelques centimètres au sud, un trou de poteau circulaire de $20 \mathrm{~cm}$ de diamètre est associé à un calage de pierre et une petite fosse.

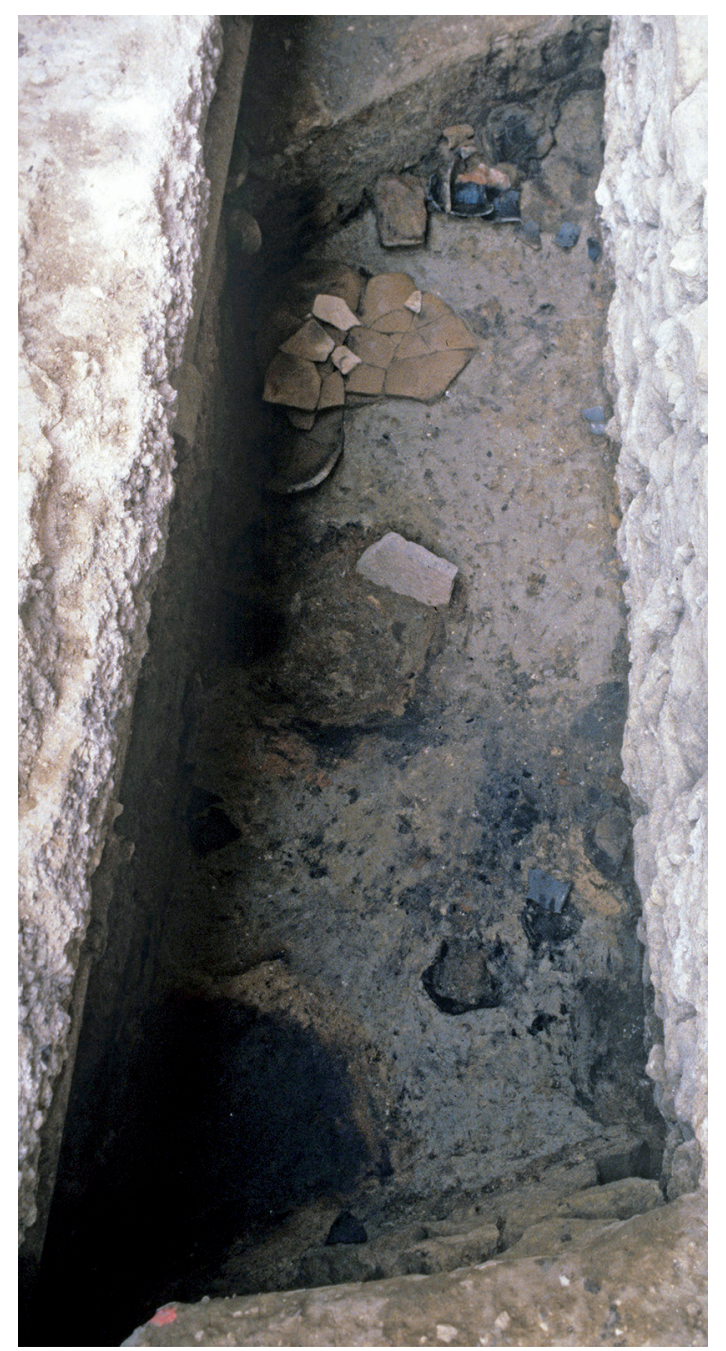

15 Sol incendié dans la maison H2 (1092), avec la plaque foyer au premier plan à gauche contre le mur MR1006, au centre le trou de poteau avec son calage, et un dolium écrasé sur place contre le mur MR1061 (photo : J. Chausserie-Laprée).
La couche de destruction (1062) recouvrant ce sol est très bien conservée et a pu être explorée avec précision malgré les conditions difficiles de la fouille en tranchée. Le sédiment qui la compose présente une configuration habituelle sur ce site: terre marron provenant des briques crues fondues recouvrant de manière plus ou moins nette un sédiment plus sableux jaune ou rubéfié appartenant à la toiture en terre. L'ensemble surmonte les restes mal identifiés, car très fragmentés ou insuffisamment carbonisés, de la charpente. Cette couche de destruction ennoie un mobilier archéologique abondant, brisé sur place par l'effondrement et/ou la fusion rapide des superstructures de l'habitation. On distingue d'une part le mobilier en céramique, écrasé sur place et retrouvé à même le sol, et d'autre part plusieurs objets en terre crue de grand format retrouvés en général au-dessus.

Ces objets, faits d'une argile crue de couleur jaune, n'ont pas subi assez les effets de la chaleur et donc d'une cuisson secondaire suffisamment élevée pour être transformés en «céramiques», même mal cuites. De ce fait, ils n'ont pu être ni prélevés ni même dessinés précisément pour en présenter les formes et en apprécier les volumes. La fouille a cependant permis d'identifier nettement au moins quatre objets en argile crue distincts au sein de cette couche de destruction: deux grandes urnes sur pied ${ }^{9}$ et un couvercle plat dans la partie est de la maison, un grand silo caréné écrasé sur le sol au centre et à l'ouest de l'espace. De tels objets en terre crue ont déjà été identifiés dans plusieurs autres habitations $\mathrm{du}$ village, en particulier dans son quartier nord-ouest (Nin 2003, 102, fig. 5; 115-117, fig. 19 et 20; 121, fig. 27 et 28) (fig. 16).

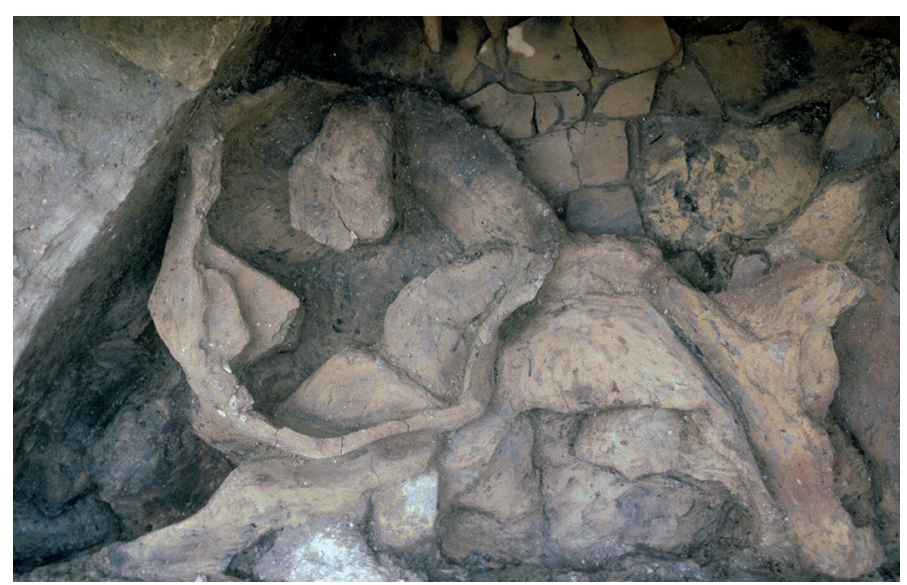

I 16 Détail des vases en terre crue dans la maison $\mathrm{H} 2$ de la période 2c (1062) (photo : J. Chausserie-Laprée). 
Les objets en céramique ne sont pas absents dans cette couche 1062: contre la paroi est, on a découvert un grand dolium à panse lisse et un pot en CNT-BER, ainsi que les fragments d'une petite jarre à panse peignée et d'une cruche grise monochrome; contre le mur ouest les tessons d'une seconde urne en céramique non tournée des rives de l'étang de Berre (fig. 41, n 11).

À travers ces nombreux vases écrasés sur place, qui associent notamment des doliums et récipients de réserve en terre crue, la maison $\mathrm{H} 2$ apparaît indéniablement comme un lieu de stockage alimentaire. Mais la fonction proprement culinaire et domestique de cette cellule est également très marquée avec l'existence d'un foyer associé à une meule en place. Sur une superficie si réduite, une telle dualité n'a rien pour surprendre si l'on considère la situation similaire observée en maints autres espaces du village (ChausserieLaprée 2005, 182).

Lors de la période $3 \mathrm{a}$, se mettent en place des niveaux de réoccupation argilo-cendreux (1058 et 1058a) qui se caractérisent par le maintien du poteau central et la présence d'aménagements associant pierres plates, dont une molette brisée en basalte (Chausserie-Laprée 1998, fig. 57), et masses argileuses. Ils sont interprétés comme des supports d'objet ou d'installation de travail domestique.

Des aménagements semblables sont attestés pour le sol argilo-cendreux 1057 (période 3b), qui vient recouvrir l'ensemble et dont la principale nouveauté est le lien avec le mur MR1056, remonté sur MR1061. Dépourvu de mobilier céramique, ce niveau a livré un hameçon en bronze (Rétif $2000, n^{\circ} 200$ ). Les sols supérieurs (1053 et 1052) comportent tous des éléments d'argile crue, de couleur jaune, orange ou grise, qui proviennent soit de vases en terre crue fondue, soit de placages muraux recouverts par les couches 1051 .

Durant sa dernière occupation (période 4), l'habitation est réorganisée et divisée en deux espaces avec la mise en place d'une cloison partielle d'axe est-ouest en terre crue massive (MR1054). Celle-ci forme un angle droit avec le mur MR1055, nouveau remontage, également en terre crue, de la paroi est de cette maison (fig. 13).

\subsubsection{La maison $\mathrm{H3}$}

A l'est, l'espace mitoyen est celui de la maison H3, délimitée par les murs MR1006 et MR1005. L'emprise de cette habitation au sein de la tranchée est très restreinte: sa largeur est inférieure à $2 \mathrm{~m}$. Le mur MR1005, qui la limite à l'ouest, est un mur de pierres liées à la terre. Les six assises en pierres calcaires conservées de ce mur révèlent des changements d'appareil, accompagnés de légères modifications d'alignement, qui indiquent qu'il s'agit une fois encore d'un empilement de plusieurs murs successifs. Ils attestent la pérennité des tracés des murs du village au fil des deux siècles d'occupation de cet habitat (fig. 17). En ce qui concerne l'habitation $\mathrm{H} 3$, comme pour la maison $\mathrm{H} 2$, la fouille de la tranchée 8904, à l'intérieur de l'église, en a révélé son contact avec la fortification, nous révélant ici son angle sud-est.

\section{Sédimentation, principaux aménagements et mobiliers} (fig. 7, section C)

Dans cette maison, l'exploration n'est pas allée au-delà du sol recouvert par la couche de destruction (1078) de la période d'incendie $2 \mathrm{c}$, faite d'une épaisse couche de terre crue sableuse rubéfiée. Elle se caractérise par l'accumulation d'un abondant mobilier de transport ou de stockage, et par la rareté dans cette partie nord de l'habitation de mobilier en terre crue et de vaisselle ${ }^{10}$. On note tout de même la présence d'une petite olpé en céramique à pate claire de Marseille (fig. 17; fig. 42, $\mathrm{n}^{\circ}$ 7). Si l'exploration de cette maison n'avait pas été si restreinte, la découverte de trois amphores massaliètes et d'un gros dolium à anses (Ratsimba 2005, pl. 29, p. 86) auraient pu donner à cette cellule un rôle spécifique de

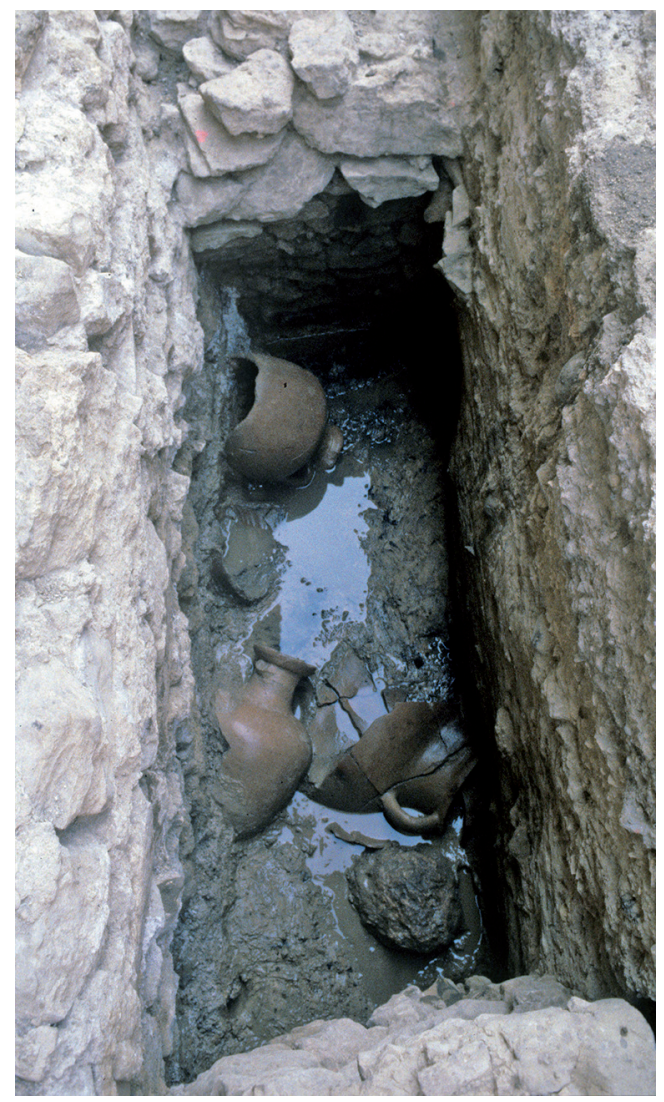

I 17 Amphores massaliètes et dolium à anses sur le niveau 1078, photo prise du mur MR1005 avec au fond le mur MR1006 caractérisé par un léger ressaut. Vue de l'est (photo : J. Chausserie-Laprée). 
cellier. Mais une telle fonction exclusive est contredite par la présence d'autres objets non céramiques. On trouve d'abord un petit mobilier métallique, constitué d'un ruban en bronze (Rétif 2000, $n^{\circ} 196$ ) et d'une alène en fer (Rétif 2000, n 197); et aussi un pilon ou aiguisoir en galet oblong présentant des traces de percussions à ses extrémités (fig. 10, nº 7; fig. 21, C), ainsi qu'une rondelle perforée taillée dans un tesson d'amphore massaliète, possible fusaïole.

Installées dans le niveau argilo-cendreux de réoccupation (1077) de cette maison (période 3a), deux petites fosses contiennent des ossements d'ovicapridés. La première, près du mur est (MR1006), présente un dépôt peu organisé, mêlé à des fragments de poteries non tournées; la seconde, creusée au pied d'une banquette basse de pierres et de tessons, près du mur ouest (1005), offre un dépôt d'os d'animaux soigneusement organisés, les os longs visiblement rangés en surface et recouvrant les os de la tête (fig. 18). Ces deux fosses sont interprétées comme des aménagements à caractère rituel (Nin, Chausserie-Laprée 2016).

Une banquette basse d'argile, reprise ensuite avec des pierres plates, est accolée au mur MR1005. Elle fonctionne en lien avec un sol de terre battue (1045), fait de passes cendreuses superposées ${ }^{11}$. Puis avec d'autres sols $(1041,1034$, 1030) de nature proche, intercalés avec des couches de brique fondue, faisant office de remblai, qui sont la marque d'une occupation longue de cette maison. On note alors la présence d'un abondant mobilier céramique, daté en particulier par la présence des premières importations italiques (bol à vernis à rosette centrale).

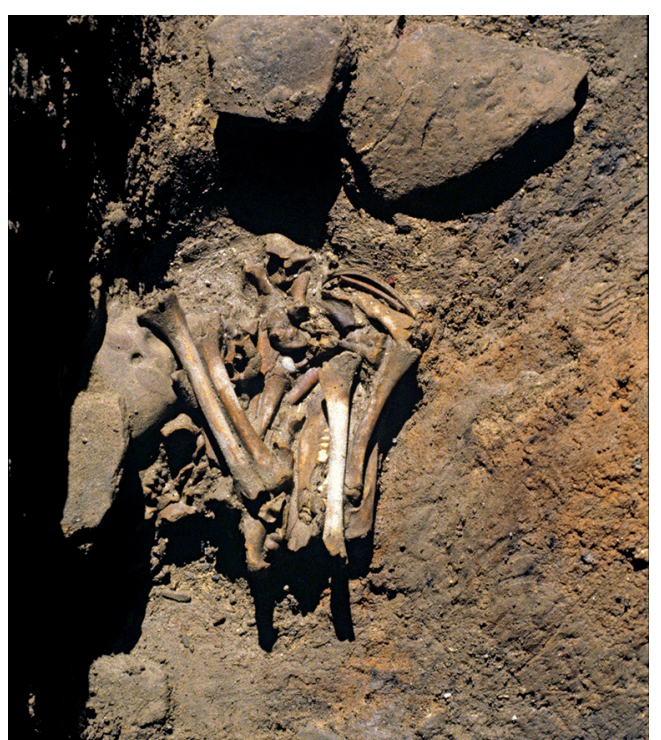

I 18 Dépôt d'ossements d'animaux dans une fosse creusée dans le niveau de sol 1077 au pied du mur MR1005. Vue du sud (photo : J. Chausserie-Laprée).
La maison est ensuite scellée par le nivellement de couches en terre crue (1015 et 1016) qui viennent se poser sur les ultimes sols d'occupation (1017, 1017a), marqués en particulier par la présence de poteries écrasées sur place et de zones d'argile rubéfiées. Ces quelques restes de la période 4 sont recouverts par les strates du second village (1007) et entamés par une fosse contemporaine dans la partie ouest de la maison.

\subsubsection{La maison $\mathrm{H4}$}

Limitée par le mur MR1005 à l'est, la maison H4 s'étend jusqu'au mur MR1027 à l'ouest, l'espace habité occupant une largeur très réduite d'environ $1,90 \mathrm{~m}$. Comme les maisons précédentes, cette habitation $\mathrm{H} 4$ adopte donc une configuration longiligne très habituelle pour les îlots simples appuyés à la fortification. La paroi ouest (MR1027) est conservée sur six assises en pierre, faite d'éléments très irréguliers, liés à la terre. À l'instar des murs MR1006 et MR1005, elle constitue l'empilement d'au moins deux murs successifs, marqués par des différences d'appareil, moellons pour les quatre premières assises, blocs pour les deux assises supérieures (fig. 19).

\section{Sédimentation, principaux aménagements et mobiliers} (fig. 7, section C)

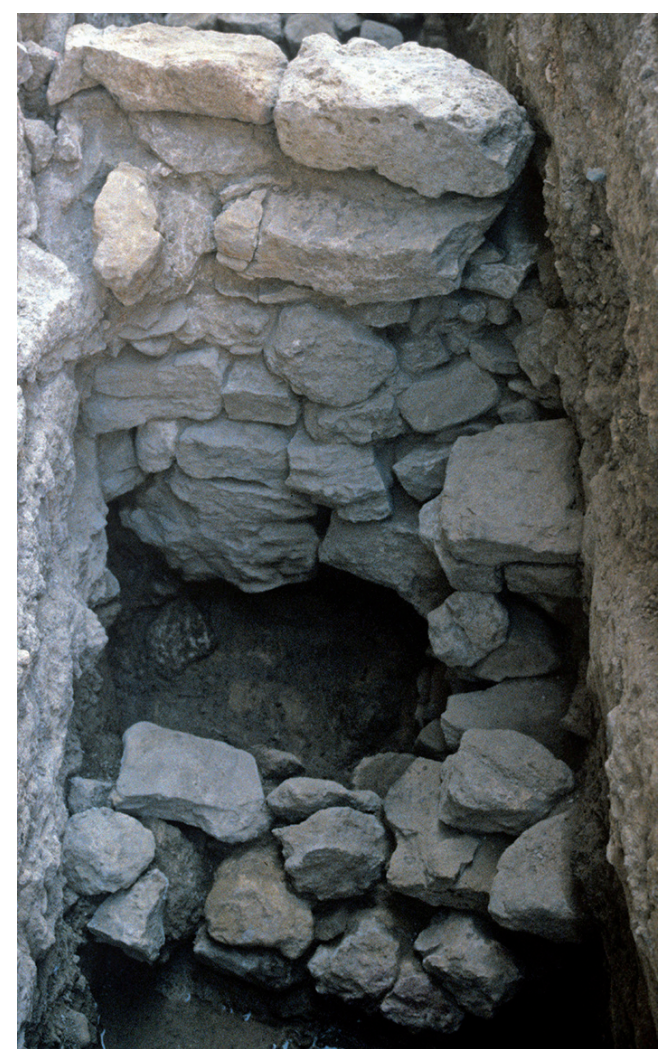

I 19 Empilement de murs formant le mur MR1027. Au premier plan le mur primitif MR1116.

Vue de l'est (photo: J. Chausserie-Laprée). 
Dans la maison $\mathrm{H} 4$, la couche de destruction par incendie du $\mathrm{IV}^{\mathrm{e}} \mathrm{s}$. av. J.-C. (période 2c) n'a été que très peu observée. Audessus d'un niveau jaune argileux présentant quelques charbons (1056), elle n'est préservée que sous la forme de deux niveaux rubéfiés (1044 et 1043), inhabituellement pauvres en mobilier. Apparaît en revanche le dispositif d'entrée de la maison, marqué ici par deux blocs de format carré, appuyés contre les murs est et ouest (fig. 20).

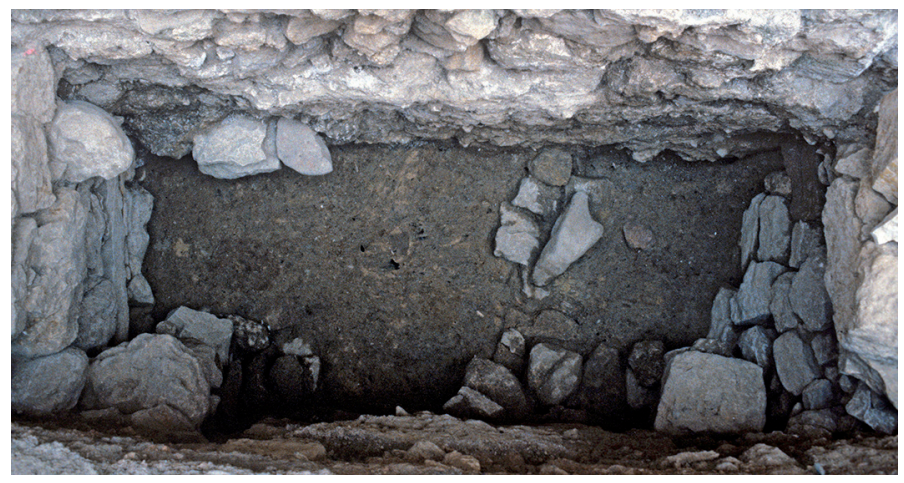

I 20 Entrée de la maison $\mathrm{H} 4$ identifiée par la présence des deux pierres contre les murs MR1005 et MR1027 marquant le seuil de la maison $\mathrm{H} 4$, au centre le début du mur antérieur MR1116. Vue du nord (photo : J. Chausserie-Laprée).

Pour la période 3a, la couche de réoccupation (1042), faite d'un remblai de terre argileuse est également pauvre en matériel, mais vient asseoir un sol d'habitat qui recèle un équipement domestique particulier (fig. 21): le regroupement, à l'angle nord-ouest de la pièce, de trois pesons laissés au sol. Tous caractérisés par le percement d'un ou deux trous, ils sont de forme et de matériau différents. L'un est fabriqué dans un tesson de mortier massaliète (fig. 10, $\mathrm{n}^{\circ} 4$ ), le deuxième fait à partir d'un fragment de dolium (fig. $10, n^{\circ} 2$ ), et le dernier réalisé dans un galet (fig. $10, n^{\circ} 3$ ). Nous interprétons ces objets percés comme des poids de pêche, bien attestés dans les habitats protohistoriques du littoral (Chausserie-Laprée 2005, 193-194). La diversité des matériaux utilisés montre un pragmatisme opportuniste pour la réalisation de ces objets. Ils font évidemment écho aux nombreux objets métalliques liés aux pratiques halieutiques des villageois, confirmés par la présence de nombreux restes ichtyologiques (Rétif 2000, 185; Chausserie-Laprée 2005, 192).

À ces poids de pêche, il faut ajouter la présence d'un jeton en céramique non tournée (fig. 10, $\mathrm{n}^{\circ} 1$ ). La fonction de ces jetons céramiques (ou rondelles) demeure obscure, malgré les nombreuses attestations pour la Protohistoire méridionale, notamment dans la région de Martigues (Verdin et al. 1996-1997, 180, fig. 15, $\mathrm{n}^{\circ} 4$; Duval 1998, 162, fig. 32, $\mathrm{n}^{\text {os }} 241$ et 242 ; Py 2006, fig. 29, n 129 , fig. 45 , n 331, fig. 59; Py 2016, 402).
Avec le recouvrement des blocs marquant le seuil de la maison, l'occupation de la période $3 \mathrm{~b}$ est marquée par la superposition de sols de terre battue cendreuse et de remblais de brique
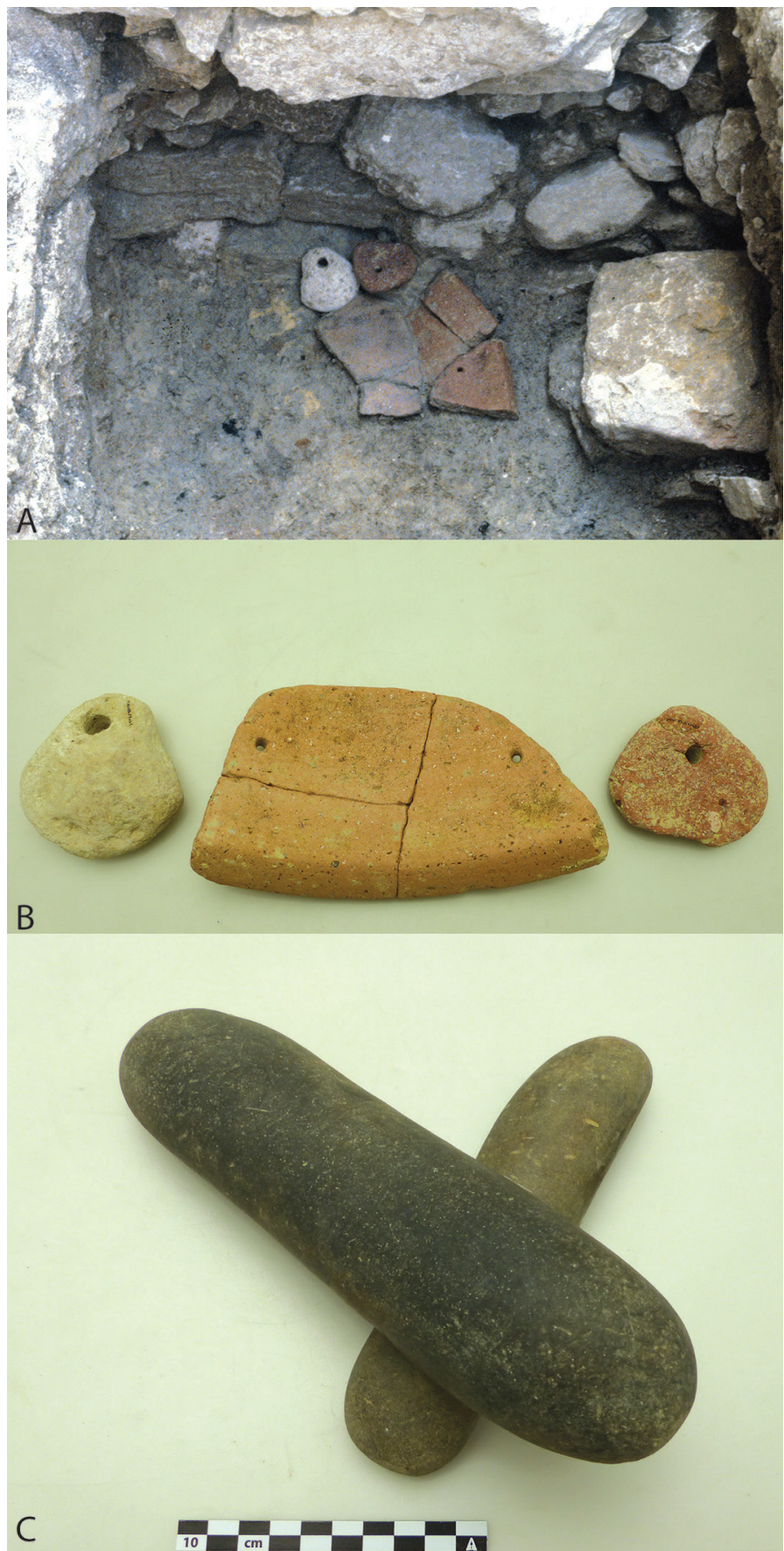

I 21 A Pesons dans leur contexte de découvertes sur le niveau de sol 1042 dans la maison H4 au pied du mur MR1027. Vue de l'est (photo: J. Chausserie-Laprée).

I B Pesons aménagés dans un galet, dans un fragment de mortier massaliète et dans un tesson de dolium (photo : Service Archéologie de la Ville de Martigues)

I C Pilons se caractérisant par leurs extrémités usées et par le polissage des zones de préhension (photo : Service Archéologie de la Ville de Martigues). 
fondue (1040, 1031 et 1028) contenant un important mobilier céramique et métallique (Rétif 2000, $\mathrm{n}^{\text {os }} 204,205,208$ ).

L'occupation ultime de cette maison (période 4) se traduit à nouveau par l'évidence d'un sol d'argile jaune (1022) auquel était associé une plaque-foyer d'argile maçonnée. Enfin, la sédimentation est surmontée par une épaisse couche de brique fondue (1021) caractéristique de la destruction du village à la fin de la période 4 .

\subsubsection{La maisons $\mathrm{H5}$}

Dans cette partie ouest de l'îlot, la maison H5 est limitée à l'ouest par le mur MR1067, assez mal conservé à cause d'une tranchée de récupération des pierres qui le composaient ${ }^{12}$. Quant à sa limite orientale, elle semble avoir évolué avec son déplacement au cours de la période 2 . Dans un premier état, elle est d'abord marquée par le mur MR1101, présent depuis l'origine, qui perd ce rôle au profit de la paroi MR1027. Dans ce second état, la maison $\mathrm{H} 5$ s'avère alors de loin la plus ample, avec une emprise est-ouest de plus de $4 \mathrm{~m}$. Par ailleurs, nous pouvons en estimer sa superficie totale à environ $20 / 25 \mathrm{~m}^{2}$, grâce aux recherches effectuées à l'intérieur de l'église. Celles-ci ont en effet mis au jour l'extrémité sud de cette maison, directement appuyée à la fortification.

\section{Sédimentation, principaux aménagements et mobiliers (fig. 7, section D)}

Ce n'est qu'à partir de la période $2 \mathrm{~b}$ que les nouveaux murs MR1067 et MR1066 des maisons H5 et H6 sont construits alors que le mur primitif MR1101 est toujours en fonction. Dans cette configuration (état 1), deux niveaux de sols superposés (1103 et 1100) marquent l'occupation de la maison H5. Ils sont signalés par des surfaces d'argile recouvertes de cendres et charbons, eux-mêmes générés par l'usage de plaques-foyers installées contre le mur ouest (MR1067). Pour l'un des foyers maçonnés associés au sol 1103 a pu être mis au jour un radier réfractaire composé de l'assemblage de tessons d'amphore de Marseille et d'un mortier de même origine cassé sur place (fig. 22).

Dans un second état, le mur MR1101 est abattu, son niveau d'arasement maintenant une sorte de marche intérieure d'environ $10 \mathrm{~cm}$ au sein de la maison $\mathrm{H} 5$, désormais agrandie d'1 $m$ vers l'est. Cette modification n'intervient que peu avant la destruction du site par incendie (période 2c). La couche de destruction (1050) s'y s'avère épaisse, mêlant terre de toiture fine jaune, plus ou moins rubéfiée, et terre à brique marron. Elle emprisonne un mobilier domestique abondant écrasé sur le sol (1094 et 1094a). Il est spécialement regroupé près d'une plaque-foyer d'argile maçon- née non loin du mur est MR1027 (fig. 23). Outre deux petites urnes en céramique non tournée, on y trouve une grande jatte recouverte par les débris d'un four complexe en terre crue $^{13}$. Mais ils ont été trop mal cuits par l'incendie pour être tous individualisés et prélevés. Seule des quatre parties qui composent habituellement ce four à avoir été clairement identifiée, un objet durci et noirci par le feu se présente sous la forme habituelle d'une grande cuve à sole perforée d'environ $0,80 \mathrm{~m}$ de diamètre (fig. 24).

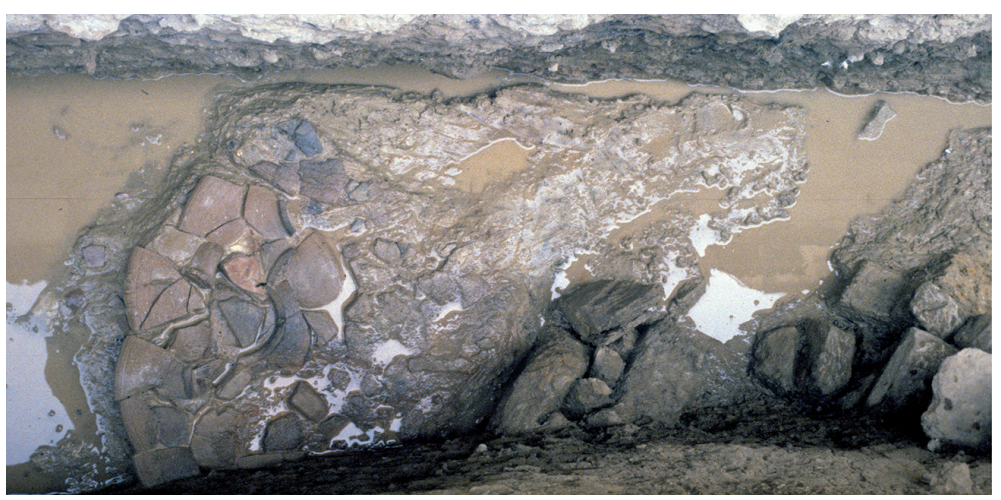

I 22 Radier d'une plaque foyer, fait de fragments d'amphores et de mortiers massaliètes sur le niveau 1103 Vue du nord (photo J. Chausserie-Laprée).

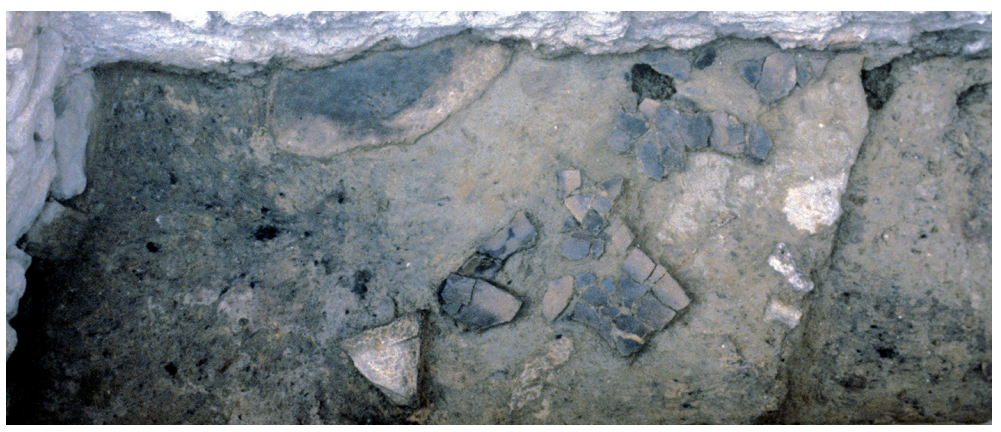

I 23 Sol incendié dans la maison H5 présentant des céramiques écrasées sur place (1094), une plaque foyer à côté du mur MR1027 et sur la droite la marche formée par le mur primitif MR1101. Vue du nord (photo : J. Chausserie-Laprée).

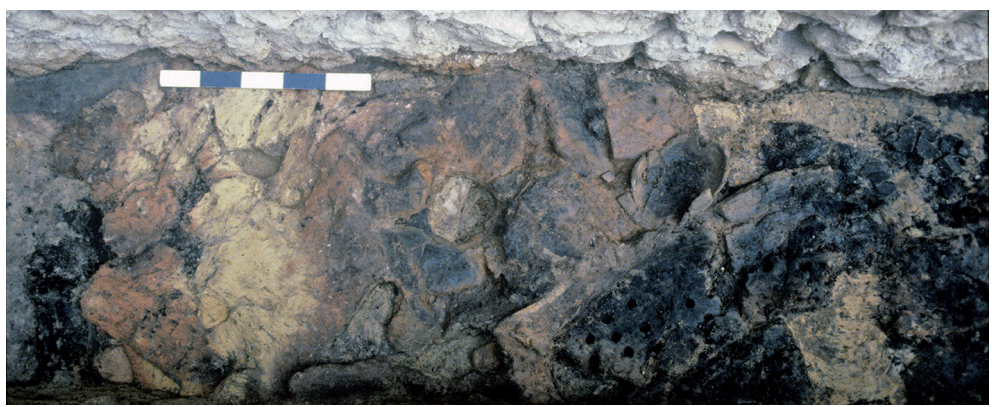

I 24 Couche d'incendie de la période 2 avec des éléments en terre crue, la sole percée d'un four complexe et les fragments des vases en céramique non tournée dans la maison H5 (1094). Vue du nord (photo : J. Chausserie-Laprée). 
On retrouve en tout cas dans cette maison $\mathrm{H} 5$ l'association couramment mise en évidence d'un four complexe et de poteries culinaires, notamment une jatte et des urnes en céramique non tournée. Mais l'absence d'indices, qu'aurait pu par exemple fournir la découverte de restes végétaux ou alimentaires associés, ne permet pas d'en donner ici la fonction particulière (Chausserie-Laprée 2005, 165). On indique enfin la découverte, à l'extrémité sud de la maison, d'une grande table de mouture incomplète en basalte. Elle présente la particularité d'avoir continué à être utilisée après que la meule a été brisée au $2 / 3$ de sa longueur initiale (Chausserie-Laprée 1998, n 54 ).

Pour les niveaux supérieurs correspondant aux périodes 3 et 4, la sédimentation des maisons H5 et H6 apparaît aussi dans la coupe de la tranchée de récupération du mur de refend MR1066.

Pour la période $3 \mathrm{a}$, au sein même de la couche de destruction inférieure, on notera l'aménagement d'un dépôt d'ossements animaux contre la paroi ouest. Il semble associé à une pierre plate taillée faisant office soit de support soit de couvercle. Il précède la constitution d'un sol (1033a), marqué par la présence de deux foyers, dont l'un est formé d'une plaque circulaire d'argile maçonnée sur un radier de tessons. Contre le mur oriental MR1027, on note aussi la présence d'un couvercle en argile crue de $50 \mathrm{~cm}$ de diamètre. Au-dessus se met en place une surface de sol argilo-cendreux (1033), dont la puissance sédimentaire est très faible.

En revanche, la destruction et l'abandon de la maison H5 (période 4) sont beaucoup mieux conservés avec une forte épaisseur de terre à brique fondue (fig. 25), voire de briques crues effondrées d'un bloc (1018) au-dessus d'un sol d'argile grise (1019) sali par l'occupation domestique de la période 3b. À sa surface, un ultime foyer d'argile, appuyé sur un radier de poteries très dégradées par le feu, est recouvert ou entouré par de nombreuses plaques d'ar-

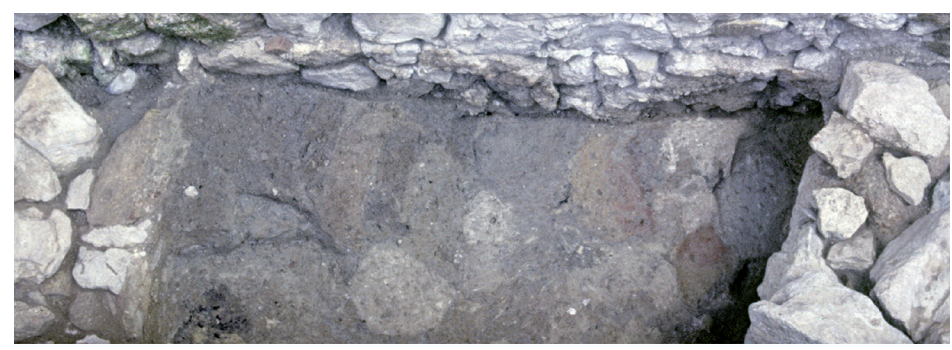

I 25 Couche hétérogène composée de brique fondue (1018) entre les murs MR1027 à gauche et MR1032 à droite Vue du nord (photo J. Chausserie-Laprée). gile appartenant à un ou plusieurs récipients en terre crue. On y trouve également une fibule en bronze à pied replié (Rétif 2000, n²09).

Il faut enfin signaler que cette maison $\mathrm{H} 5$ du premier village est l'une des seules de la tranchée 8903 à être surmontée par une construction appartenant sans conteste à l'organisation du second village gaulois, en l'occurrence le mur MR1032, d'axe nord-sud (fig. 25).

\subsubsection{La maison $\mathrm{H6}$}

Au-delà et à l'ouest du mur MR1067, en partie récupéré, se développe l'emprise de la maison H6. Le sondage 8903 la montre coupée par le large fossé 1085 , qui nous prive de toute sédimentation protohistorique jusqu'à sa probable limite ouest originelle, à savoir la muraille du village, MR1117, mentionnée plus haut. La restitution de la configuration initiale de la maison H6 demeure incertaine, aussi bien pour son emprise est-ouest que pour son découpage intérieur. Pour le premier point, rien n'interdit en effet de penser que l'îlot $\mathrm{H}$ n'ait pas été divisé davantage encore, avec la présence, à l'est d'une étroite maison H6, d'une cellule longiligne supplémentaire H7, qui aurait été appuyée contre la fortification. Le creusement du fossé 1085 nous empêche de le savoir.

Quant à la partition nord-sud de la maison H6, suggérée par la présence du mur de cloisonnement MR1066, qui s'enfonce dans la coupe de terrain nord du sondage 8903, on ne sait pas non plus quelle est sa vraie nature: simple cloison intermédiaire divisant la maison en deux pièces communicantes, ou séparation plus franche en deux habitations indépendantes (H6 au sud, H7 au nord) donnant sur une voie ou une place située à l'ouest.

Sédimentation, principaux aménagements et mobiliers (fig. 7, section D)

Au sein du fragment de la maison H6 que nous connaissons, on retrouve à peu près le même schéma stratigraphique que dans la maison précédente. De la période $2 \mathrm{~b}$ qui précède la destruction par incendie du village, n'a été fouillé qu'un seul niveau d'habitat 1105 , fait d'une surface argileuse recouverte de dépôts cendreux. Une petite épaisseur de sable et de brique fondue la recouvre pour servir d'assise au sol d'argile jaune (1102).

C'est à ce niveau qu'a été explorée l'épaisse couche 1070 qui marque ici la destruction violente du village (période $2 \mathrm{c}$ ) (fig. 26). Sur à peine $2 \mathrm{~m}^{2}$, la fouille a permis de dégager et de comprendre une part de l'aménagement et du mobilier présent à l'angle nord-est de la pièce H6. Le sol est parsemé de tessons de céramique appartenant à une amphore de 
Marseille et à plusieurs doliums. On observe aussi un remarquable tesson décoré à la peinture ocre, appartenant à une petite jarre en céramique non tournée à pate claire (fig. 42, $\mathrm{n}^{\circ} 10$ ). Les récipients et objets en terre crue ne sont pas non plus absents, avec au moins un grand silo cylindrique accompagné peut-être de son couvercle ${ }^{14}$.

Non loin d'une dalle calcaire taillée, parfaitement en place et faisant office de banc ou de support d'activité, on a mis également au jour deux galets oblongs utilisés comme pilon et/ou aiguisoir.

Comme celui découvert dans la maison $\mathrm{H} 3$ (fig. 10, $\mathrm{n}^{\circ}$ 6), ces pilons, nommés aussi «concasseurs en pierre», sont bien attestés en Gaule méditerranéenne et sont identifiables par les

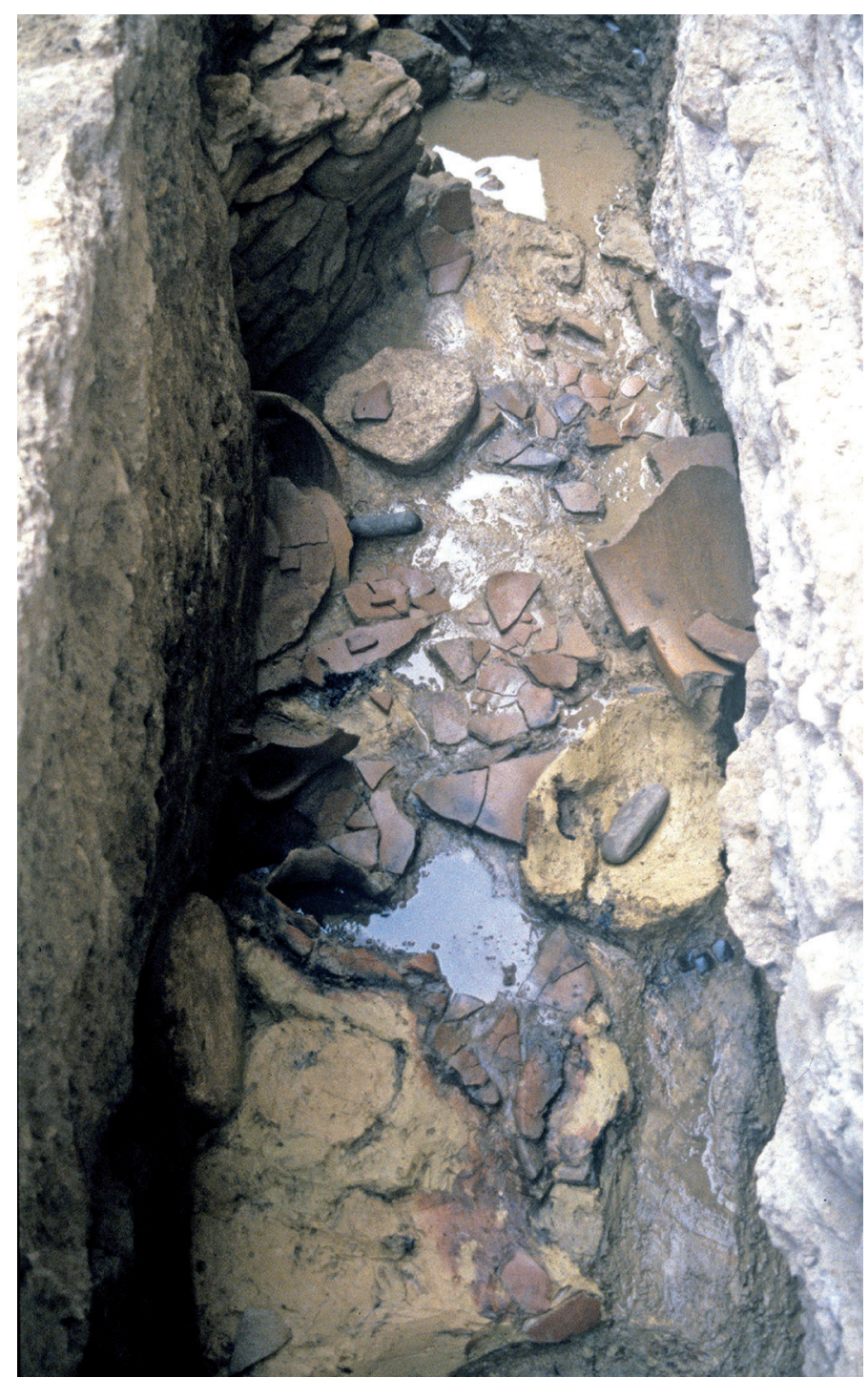

I 26 Couche d'incendie de la période 2c de la maison $\mathrm{H} 6$ avec les fragments de doliums écrasés sur place et des éléments de vase en terre crue (US 1070 et 1102).

Vue de l'ouest (photo: J. Chausserie-Laprée). traces d'usure et de percussion localisées aux extrémités et par le polissage des zones de préhension (Curé 2013, 285; Py 2016, 256). À Lattes, Stéphanie Raux $(1999,447)$ leur attribue un usage varié: concassage de minerai ou de matières minérales dures (calcite, coquillages) pour servir de dégraissant dans la fabrication de la céramique; aiguisoir, etc.

L'équipement domestique est complété par une plaquefoyer d'argile maçonnée sur laquelle reposait, curieusement de chant, une pierre taillée ronde de $40 \mathrm{~cm}$ de diamètre.

$\mathrm{Au}$-dessus de la couche de destruction, l'espace est rapidement réoccupé, la maison H6 montrant l'aménagement d'un sol argileux (1069) couvert en surface d'un abondant mobilier en céramique et en terre crue. Une nouvelle pierre plate taillée est soigneusement calée dans le sol, comme support, mais dont la fonction domestique ou architecturale précise demeure indéterminée. Lors de la destruction finale de l'habitat (période 4), le dernier sol d'occupation (1068) pourvu du même type d'aménagement (pierre de support), est recouvert d'une épaisse couche de brique fondue (1018 ouest) emprisonnant à la base pierres en vrac et tessons de dolium.

\subsubsection{Le secteur ouest}

Pour ce secteur situé à l'extérieur des remparts, cette partie de la tranchée 8903 est, à l'instar du secteur oriental, difficile à appréhender. Il est délimité à l'est par des blocs de pierre, interprétés comme la tour d'angle du rempart, largement entamée par le fossé contemporain 1085. Quant à la limite ouest qui correspond à l'extension maximale de notre investigation, elle nous est donnée par un mur protohistorique MR1114, d'axe nord-sud, auquel est peut-être associé, au sud, un muret en retour MR1112 (fig. 8). Alors qu'il est bâti sans doute dès le début du $\mathrm{IV}^{\mathrm{e}} \mathrm{s}$. av. J.-C. (période 2b), MR1114 est exactement surmonté par le soubassement d'un mur cimenté d'époque contemporaine. Appartenant à une construction de bonne facture, le mur gaulois est constitué d'un solin de quatre assises bien appareillées de pierres liées à la terre ${ }^{15}$. À $4 \mathrm{~m}$ de la tour d'angle sud-ouest du rempart, il vient délimiter de manière nette une zone hors les murs dont la fonction varie au fil de l'occupation.

\section{Sédimentation, principaux aménagements et mobiliers (fig. 7, section E)}

On a vu plus haut que c'est dans cette zone que les strates et structures relatives aux premiers temps (période 1 et $2 \mathrm{a}$ ) de l'occupation et de la fortification du site gaulois étaient le mieux préservées. Après la mise en place de la fortification, puis sa destruction partielle, ce secteur reste d'abord principa- 
lement réservé à l'épandage des déchets domestiques. C'est au sein des sédiments (1098 et 1095) eux-mêmes très charbonneux et lités, contenant de nombreux tessons de céramiques et restes de vases en terre crue que le mur MR1114 est encore bâti. Dans la zone la plus proche du rempart, on rencontre encore une grosse accumulation de blocs (1096), restes de l'effondrement de la tour ou blocage du renfort MR1097.

Une deuxième phase de la sédimentation se traduit par la mise en place, au sein d'un remblai de terre à brique (1089), d'un massif de grosses pierres (MR1087) d'environ 0,80 m de large. À peine maçonné, et parallèle à la muraille principale, il divise l'espace exploré en deux moitiés égales, sans que sa fonction ne soit clairement établie. Cet amas médian de blocs vient gêner la lecture d'une épaisse sédimentation (1079, 1080, 1081, 1084) assez hétérogène propre à la destruction (ici sans incendie) et à la fusion des superstructures en pierre et en terre, qui surmontaient les murs MR1112 et MR1114 et peut-être aussi la muraille (fig. 27). L'ensemble masque le sol d'habitat proprement dit de la période 2c (1088), parfaitement identifié par sa texture, une pellicule d'argile jaune très peu salie, et surtout par son association avec une belle plaquefoyer d'argile maçonnée de forme ovale. Celle-ci est appuyée directement contre le rempart et recouverte de tessons d'une urne non tournée des rives de l'étang de Berre (fig. 28).

Enfin, cet espace domestique bâti extra-muros ne semble pas avoir conservé la moindre trace d'occupation des périodes les plus récentes du premier village de l'île excepté les lambeaux 1082 et 1083 .

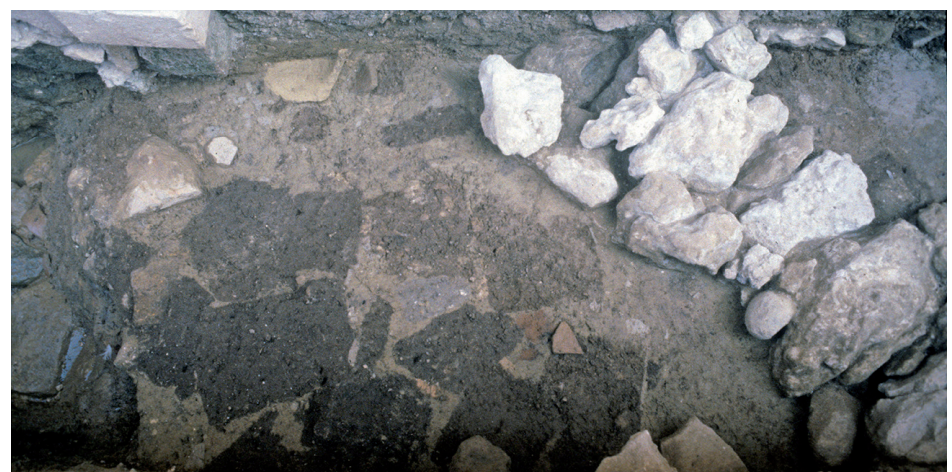

I 27 Niveaux argileux englobant des briques posées à plat, parfois de chant (1081) auprès d'une structure en pierre indéterminée (1087) dans le secteur ouest.

Vue du nord (photo : J. Chausserie-Laprée).

\section{Les types céramiques du premier village de Martigues}

Le matériel étudié dans cet article est composé de 4329 fragments et de 329 individus dont 249 bords (fig. 29). Il apparaît inégalement réparti chronologiquement puisque plus d'un tiers des fragments provient de la période $2 \mathrm{c}$ d'incendie qui a livré des contextes bien plus riches que toute autre période dans ces tranchées. Le mobilier ${ }^{16}$ sera présenté par catégorie et par période puisque l'intérêt de cette étude est de livrer des contextes cohérents et bien datés grâce aux séquences stratigraphiques identifiées sur le site.

\subsection{La période 1 (440-425 av. J.-C.)}

La période 1 correspond à l'installation du village et à sa première destruction au troisième quart du $\mathrm{V}^{\mathrm{e}} \mathrm{s}$. av. J.-C. Le plan définitif de l'îlot $\mathrm{H}$ n'est pas encore mis en place et la céramique de la période 1 est bien représentée malgré le peu d'US rattachées à cette période dans le sondage 8903. Elle comprend un total de 319 fragments et de 29 individus (fig. 30).

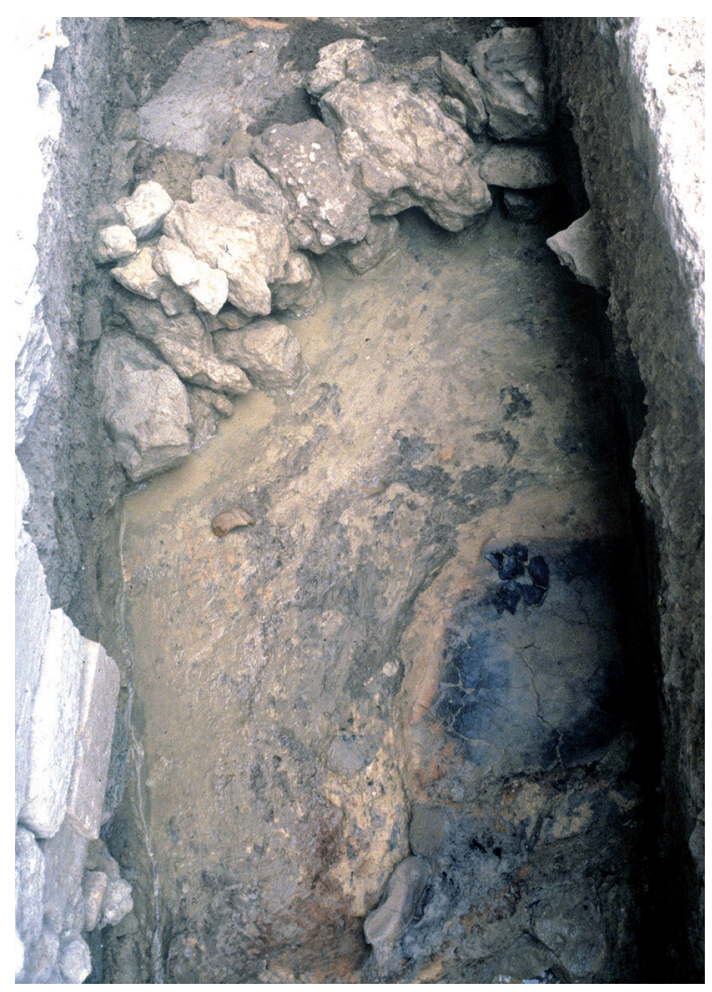

28 Sol d'habitat (1088) avec une plaque foyer d'argile maçonnée. Au fond, l'aménagement en pierre (1087). Vue de l'est (photo : J. Chausserie-Laprée). 


\begin{tabular}{|l|c|c|c|c|c|c|c|c|c|}
\hline \multirow{2}{*}{ catégorie } & \multicolumn{4}{|c|}{ NFR } & \multicolumn{3}{c|}{ NMI } & \multicolumn{3}{c|}{ NBD } \\
\cline { 2 - 11 } & $\mathrm{nb}$ & $\% /$ tot & $\% / g r p$ & $\mathrm{nb}$ & $\% /$ tot & $\% / g r p$ & $\mathrm{nb}$ & $\% /$ tot & $\% / g r p$ \\
\hline gris mono & 100 & 2,31 & 3,59 & 24 & 7,29 & 8,51 & 18 & 7,23 & 8,18 \\
\hline cl.-mas & 657 & 15,18 & 23,57 & 71 & 21,58 & 25,18 & 50 & 20,08 & 22,73 \\
\hline attique & 1 & 0,02 & 0,04 & 1 & 0,3 & 0,35 & & & \\
\hline pseudo-at & 7 & 0,16 & 0,25 & 4 & 1,22 & 1,42 & 1 & 0,4 & 0,45 \\
\hline petest & 17 & 0,39 & 0,61 & 4 & 1,22 & 1,42 & 4 & 1,61 & 1,82 \\
\hline camp-a & 19 & 0,44 & 0,68 & 6 & 1,82 & 2,13 & 4 & 1,61 & 1,82 \\
\hline ct-ind & 1 & 0,02 & 0,04 & 1 & 0,3 & 0,35 & & & \\
\hline TOURNÉE FINE & 802 & 18,52 & 28,78 & 111 & 33,73 & 39,36 & 77 & 30,93 & 35 \\
\hline com-mas & 61 & 1,41 & 2,19 & 16 & 4,86 & 5,67 & 13 & 5,22 & 5,91 \\
\hline $\begin{array}{l}\text { TOURNÉE } \\
\text { COM. }\end{array}$ & 61 & 1,41 & 2,19 & 16 & 4,86 & 5,67 & 13 & 5,22 & 5,91 \\
\hline CNT-Pro & 1792 & 41,4 & 64,28 & 133 & 40,43 & 47,16 & 110 & 44,18 & 50 \\
\hline CNT-Ber & 99 & 2,29 & 3,55 & 13 & 3,95 & 4,61 & 12 & 4,82 & 5,45 \\
\hline CNT-Cl & 32 & 0,74 & 1,15 & 7 & 2,13 & 2,48 & 7 & 2,81 & 3,18 \\
\hline CNT-Gr & 1 & 0,02 & 0,04 & 1 & 0,3 & 0,35 & & & \\
\hline CNT-Ind & 1 & 0,02 & 0,04 & 1 & 0,3 & 0,35 & 1 & 0,4 & 0,45 \\
\hline NON TOURNÉE & 1925 & 44,47 & 69,06 & 155 & 47,11 & 54,95 & 130 & 52,21 & 59,08 \\
\hline VAISSELLE & $\mathbf{2 7 8 8}$ & $\mathbf{6 4 , 4}$ & & $\mathbf{2 8 2}$ & $\mathbf{8 5 , 7}$ & & $\mathbf{2 2 0}$ & $\mathbf{8 8 , 3 6}$ & \\
\hline a-mas & 678 & 15,66 & 100 & 24 & $\mathbf{7 , 2 9}$ & 100 & 13 & 5,22 & 100 \\
\hline AMPHORES & $\mathbf{6 7 8}$ & $\mathbf{1 5 , 6 6}$ & & $\mathbf{2 4}$ & $\mathbf{7 , 2 9}$ & & $\mathbf{1 3}$ & $\mathbf{5 , 2 2}$ & \\
\hline dolium & $\mathbf{8 6 3}$ & $\mathbf{1 9 , 9 4}$ & & $\mathbf{2 3}$ & $\mathbf{6 , 9 9}$ & & $\mathbf{1 6}$ & $\mathbf{6 , 4 3}$ & \\
\hline TOTAL & $\mathbf{4 3 2 9}$ & & & $\mathbf{3 2 9}$ & & & $\mathbf{2 4 9}$ & & \\
\hline
\end{tabular}

29 Données quantitatives cumulées de toutes les phases d'occupation de l'îlot H du sondage 8903 du premier village.

\begin{tabular}{|c|c|c|c|c|c|c|c|c|c|c|c|c|c|}
\hline \multirow{2}{*}{ catégorie } & \multicolumn{3}{|c|}{ NFR } & \multicolumn{3}{|c|}{ NMI } & \multicolumn{3}{|c|}{ NBD } & \multirow{2}{*}{ forme } & \multirow{2}{*}{ type } & \multirow{2}{*}{$\begin{array}{l}\text { éléments } \\
\text { représentés }\end{array}$} & \multirow{2}{*}{ illustrations } \\
\hline & $\mathrm{nb}$ & $\% /$ tot & $\% / g r p$ & $\mathrm{nb}$ & $\% /$ tot & $\% / g r p$ & $\mathrm{nb}$ & $\% /$ tot & $\% / g r p$ & & & & \\
\hline gris mono & 5 & 1,57 & 2,13 & 3 & 10,34 & 13,04 & 2 & 9,09 & 11,11 & $\begin{array}{l}\text { coupe carénée } \\
\text { coupe } \\
\text { askos }\end{array}$ & $\begin{array}{l}\text { GR-MONO } 3 \\
\text { GR-MONO } 5 \\
\text { GR-MONO ind. }\end{array}$ & $\begin{array}{l}1 \mathrm{~b} \\
1 \mathrm{~b} \\
1 \mathrm{a}\end{array}$ & fig. $31, n^{\circ} 21$ \\
\hline cl.-mas & 48 & 15,05 & 20,43 & 6 & 20,69 & 26,09 & 5 & 22,73 & 27,78 & $\begin{array}{l}\text { coupe carénée } \\
\text { cruche } \\
\text { autre } \\
\text { olpé }\end{array}$ & $\begin{array}{l}\text { CL-MAS } 222 \\
\text { CL-MAS ind. } \\
\text { CL-MAS ind. } \\
\text { CL-MAS ind. }\end{array}$ & $\begin{array}{l}1 \mathrm{~b} \\
1 \mathrm{a} \\
3 \mathrm{~b} \\
1 \mathrm{~b}\end{array}$ & $\begin{array}{l}\text { fig. } 31, n^{\circ} 15 \\
\text { fig. } 31, n^{\circ s} 17-19 \\
\text { fig. } 31, n^{\circ} 16\end{array}$ \\
\hline TOURNÉE FINE & 53 & 16,62 & 22,56 & 9 & 31,03 & 39,13 & 7 & 31,82 & 38,89 & & & & \\
\hline CNT-Pro & 167 & 52,35 & 71,06 & 10 & 34,48 & 43,48 & 7 & 31,82 & 38,89 & \begin{tabular}{|l} 
coupe \\
urne \\
coupe
\end{tabular} & $\begin{array}{l}\text { CNT-PRO C3 } \\
\text { CNT-PRO ind. } \\
\text { CNT-PRO ind. } \\
\text { CNT-PRO U4 }\end{array}$ & $\begin{array}{l}1 b \\
1 b, 1 f \\
1 b \\
4 b, 2 d\end{array}$ & $\begin{array}{l}\text { fig. } 31, n^{\circ} 9 \\
\text { fig. } 31, n^{\circ} 3 \\
\text { fig. } 31, n^{\text {os }} 1,2, \\
4,5,6,7\end{array}$ \\
\hline VAISSELLE & 235 & 73,67 & & 23 & 79,3 & & 18 & 81,83 & & & & & \\
\hline a-mas & 46 & 14,42 & 100 & 3 & 10,34 & 100 & 1 & 4,55 & 100 & $\begin{array}{l}\text { amphore } \\
\text { amphore }\end{array}$ & $\begin{array}{l}\text { A-MAS bd5 } \\
\text { A-MAS ind. }\end{array}$ & $\begin{array}{l}1 \mathrm{~b} \\
1 \mathrm{f}\end{array}$ & $\begin{array}{l}\text { fig. } 31, n^{\circ} 23 \\
\text { fig. } 31, n^{\circ} 22\end{array}$ \\
\hline AMPHORES & 46 & 14,42 & & 3 & 10,34 & & 1 & 4,55 & & & & & \\
\hline dolium & 38 & 11,91 & & 3 & 10,34 & & 3 & 13,64 & & \begin{tabular}{|l|} 
dolium \\
dolium \\
dolium \\
dolium \\
\end{tabular} & $\begin{array}{l}\text { DOLIUM bd5 } \\
\text { DOLIUM bd8c } \\
\text { DOLIUM bd8d } \\
\text { DOLIUM ind. }\end{array}$ & $\begin{array}{l}1 \mathrm{~b} \\
1 \mathrm{~b} \\
1 \mathrm{~b} \\
1 \mathrm{~d} \\
\end{array}$ & $\begin{array}{l}\text { fig. } 31, n^{\circ} 26 \\
\text { fig. } 31, n^{\circ} 25 \\
\text { fig. } 31, n^{\circ} 24\end{array}$ \\
\hline TOTAL & 319 & & & 29 & & & 22 & & & & & & \\
\hline
\end{tabular}

I 30 Période 1 (450-425 av. J.-C.) - Données quantitatives et typologiques du mobilier. 


\subsubsection{Céramique non tournée provençale}

(fig. 31, $n^{o s} 1$ à 10)

Constituant la majorité du corpus, la céramique non tournée provençale présente une grande variété de pâtes plus ou moins denses, aux couleurs multiples, parfois sur un même individu. Le dégraissant est souvent bien visible, composé de particules de calcite et souvent d'éléments cristallins sans doute du quartz.

Les urnes sont les plus courantes (fig. 31 , $\mathrm{n}^{\text {os }} 1-5$ ) et sont souvent de type CNT-PRO U4 correspondant à des urnes à l'épaulement peu marqué, au fond plat (fig. $31, n^{\circ} 8$ ), parfois décorées de fines incisions. Il faut noter la présence de deux bords d'urne à pâte orangée/rouge décorée par peignage léger réalisé sur la panse et remontant jusque sous la lèvre. Ces deux urnes ne ressemblent pas aux habituelles productions martégales (fig. $31, \mathrm{n}^{\text {os }} 4-5$ ). On relève aussi la présence de vases pourvus de décor à chevrons (fig. $31 \mathrm{n}^{\text {os }} 6$ et 7 ).

Des éléments de coupes sont aussi présents (fig. 31, $\mathrm{n}^{\circ} 9$ et 10), parmi lesquels un bord de coupe à panse carénée, CNTPRO C3, bien polie: sa pâte est grise et révèle peu d'inclusions par rapport aux urnes (fig. 31, $\mathrm{n}^{\circ}$ 9).

\section{Céramique non tournée des rives de l'étang de Berre}

(fig. 31, $n^{o s} 11$ à 13)

Les productions de l'étang de Berre sont également attestées. Reconnaissables à leur finesse et à leur pâte bien cuite et homogène, elles sont presque toujours noires ou grises. Leur paroi, soigneusement polie et peignée, leur confère un certain éclat qu'on ne retrouve pas dans les productions provençales (fig. $31, \mathrm{n}^{\text {os }} 11-13$ ).

\subsubsection{Céramique non tournée à pâte claire}

(fig. 31, $n^{\circ} 14$ )

Une grande urne à pâte claire non tournée de type CNT-CL U5 avec la lèvre à l'horizontale est attestée. Sa pâte jaune clair très épurée rappelle la céramique claire massaliète, mais l'intérieur de l'urne montre de nombreuses bosses et creux prouvant le montage au colombin. Quelques traces brunes recouvrent la lèvre et la panse et indiquent, peutêtre, la présence d'un engobe.

La céramique non tournée à pâte claire est une production céramique qui semble utiliser une argile proche de celle des productions claires massaliètes, tout en conservant des formes et des techniques locales. Elle est attestée dans la région ouest de l'étang de Berre puisqu'elle est identifiée par F. Marty au Castellan à Istres avec deux formes d'urne et une coupe (Marty 2002, fig. 9, $\mathrm{n}^{\text {os }} 80,81$ et 82 ). La plus ancienne attestation de cette catégorie céramique provient de la ferme-grenier de Coudouneù à Lançon (Verdin et al. 1996-1997, fig. 15, nº 6). Elle est aussi présente à Martigues dans l'îlot A au nord du village (Damotte 2003, 19, $\mathrm{n}^{\circ}$ 9) durant la période $2 \mathrm{a}$ ainsi que dans les couches de destruction du IV $\mathrm{V}^{\mathrm{e}}$ s. av. J.-C. (Chausserie-Laprée et al. 1984, 77).

\subsubsection{Céramique à pâte claire massaliète}

(fig. 31, $n^{o s} 15$ à 19)

Pour la céramique à pâte claire massaliète, qui est ici très fragmentaire, l'élément le mieux conservé est un bord de coupe carénée de type CL-MAS 222 à parois verticales rectilignes à lèvre légèrement biseautée. Des traces brunes sur la paroi interne peuvent faire penser à de la peinture (fig. 31, $\mathrm{n}^{\circ} 15$ ). Il s'agit d'une forme de coupe assez courante parmi les productions marseillaises qu'on retrouve sur certains sites dès la fin du $\mathrm{V}^{\mathrm{e}} \mathrm{s}$. av. J.-C. notamment à Espeyran vers 400 av. J.-C. (Barruol, Py 1978, 83, fig. 47, $\left.n^{\circ} 33\right)$.

Enfin, quatre bords, trop petits pour être caractérisés renvoient peut-être à des petits lécythes ou de petites olpés (fig. $31, \mathrm{n}^{\text {os }} 16$ à 19).

\subsubsection{Dolium (fig. 31, $n^{o s} 24$ à 26)}

Trois bords de doliums présentent une pâte souvent claire ou rosée avec un dégraissant bien visible à l'œil nu composé principalement de calcite et de quartz. Leur diamètre est supérieur à $40 \mathrm{~cm}$, phénomène courant pour les périodes anciennes du site (Damotte 2003, fig. 16, $\mathrm{n}^{\text {os }} 2-3$ ). Le premier, de type bd8d, est à lèvre amincie avec un méplat légèrement incliné vers l'intérieur (fig. $31, n^{\circ} 24$ ). Un deuxième bord de type bd8c se caractérise par sa couleur jaune clair aux nombreuses inclusions de chaux et sa pâte grise (fig. 31, $\mathrm{n}^{\circ} 25$ ). Le dernier, de type bd5, à méplat incliné vers l'intérieur est très massif, sa couleur est plus orangée, avec de nombreuses inclusions de chaux (fig. 13, $\mathrm{n}^{\circ} 26$ ).

\subsubsection{Amphore (fig. 31, $n^{\text {os }} 22$ et 23)}

Les fragments d'amphores massaliètes sont assez nombreux (environ $15 \%$ des fragments) cependant seul un bord A-MAS bd5 est identifié ainsi qu'un fond (fig. 31, $\mathrm{n}^{\text {os }} 22$ et 23 ).

\subsection{7. Élément en terre crue (fig. 31, $n^{\circ} 27$ )}

Des éléments en terre crue ont été découverts et partiellement prélevés durant la fouille. Un unique élément de forme a été identifié pour la période 1. Il s'agit sans doute d'un fragment d'un pied de vase de stockage présentant des traces de fibres végétales sur ses parois. 
)
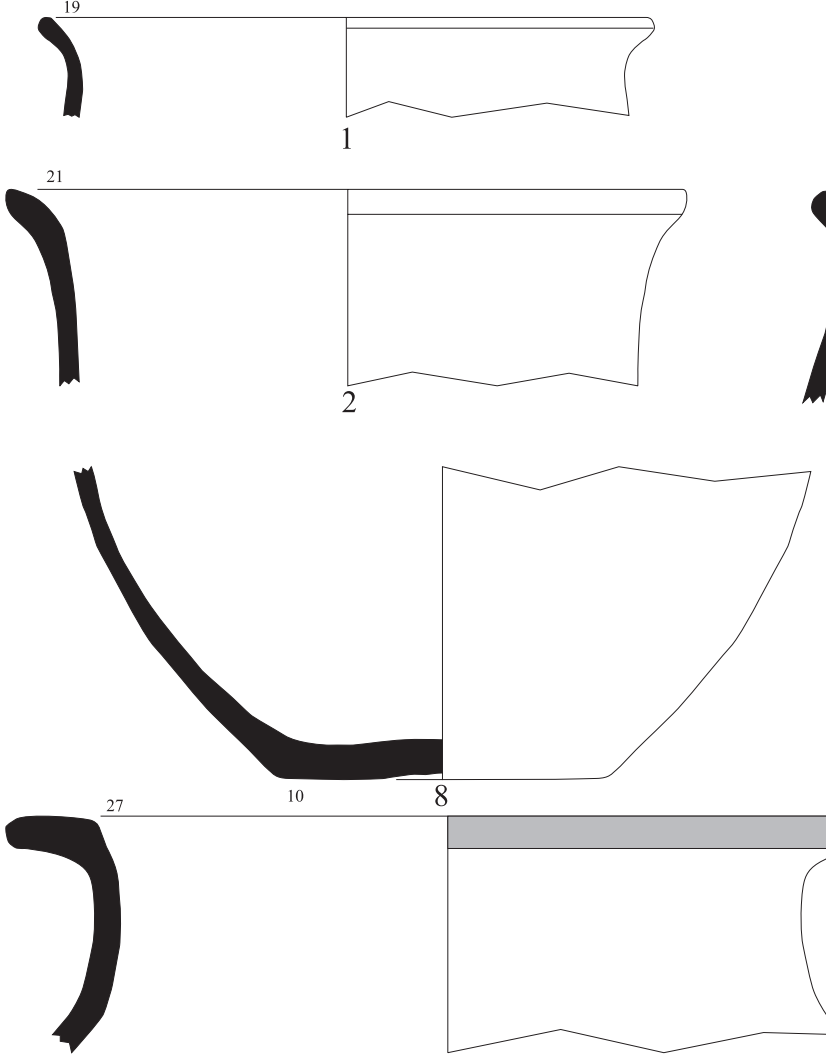

$0^{13}$

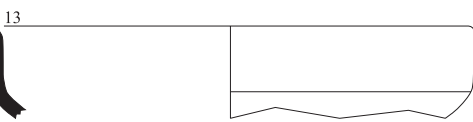

15

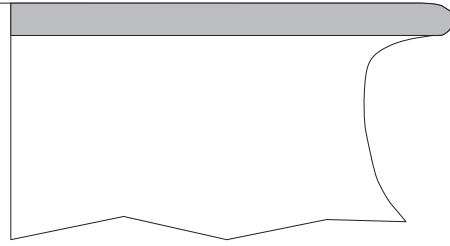

14

16
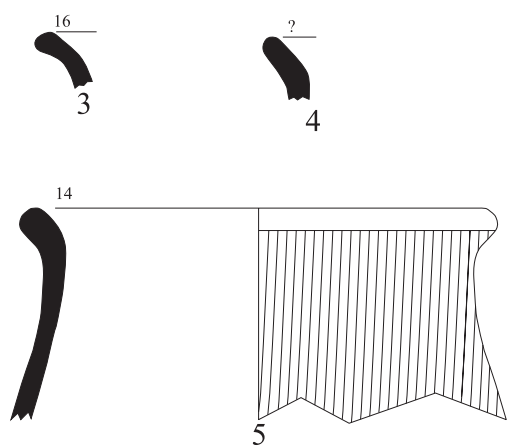
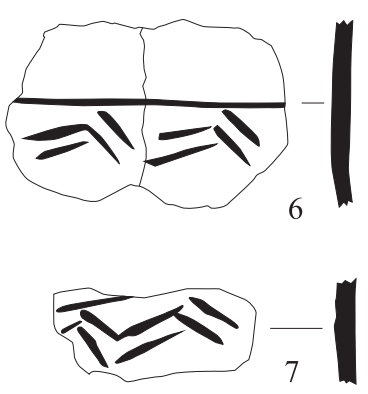
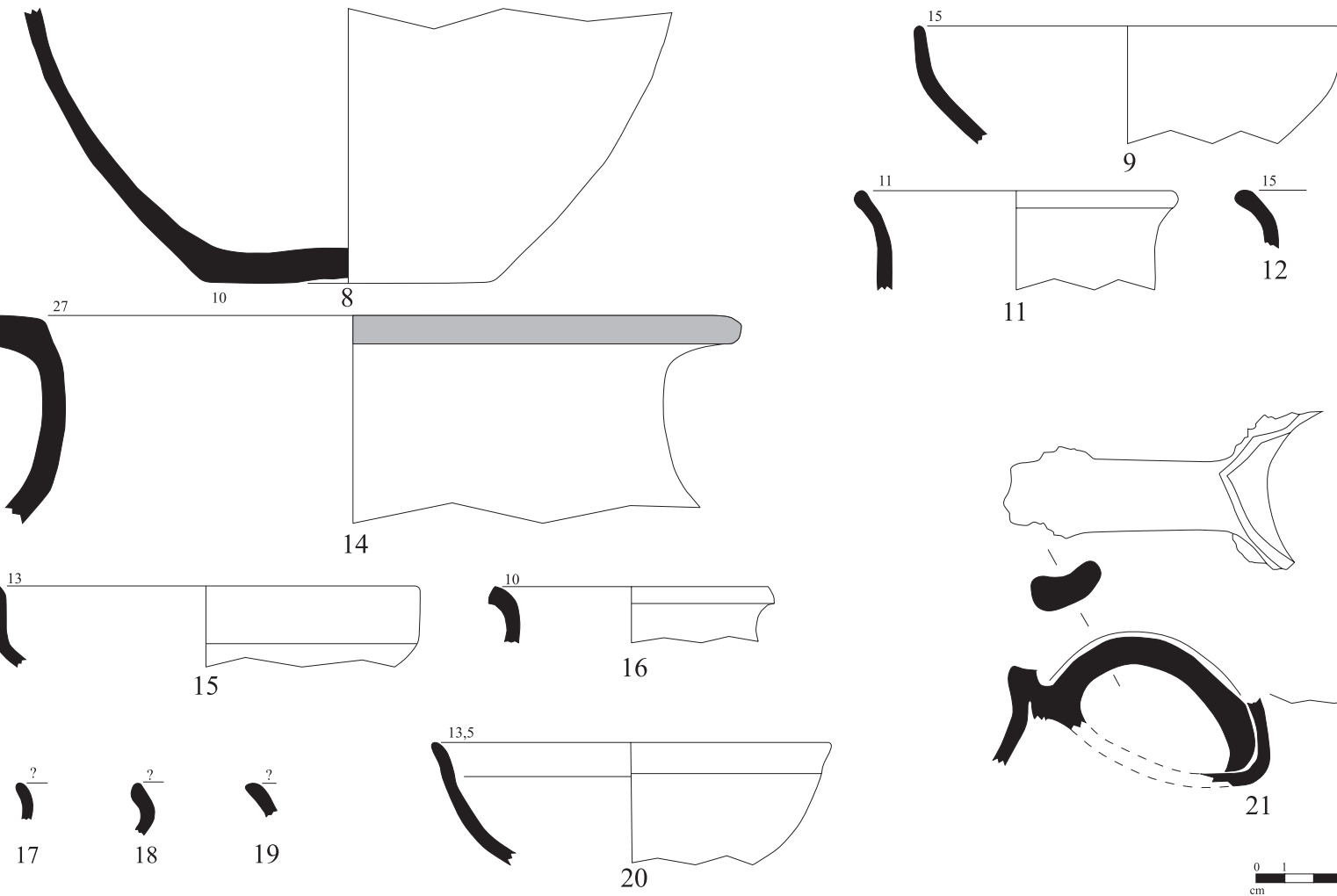

11
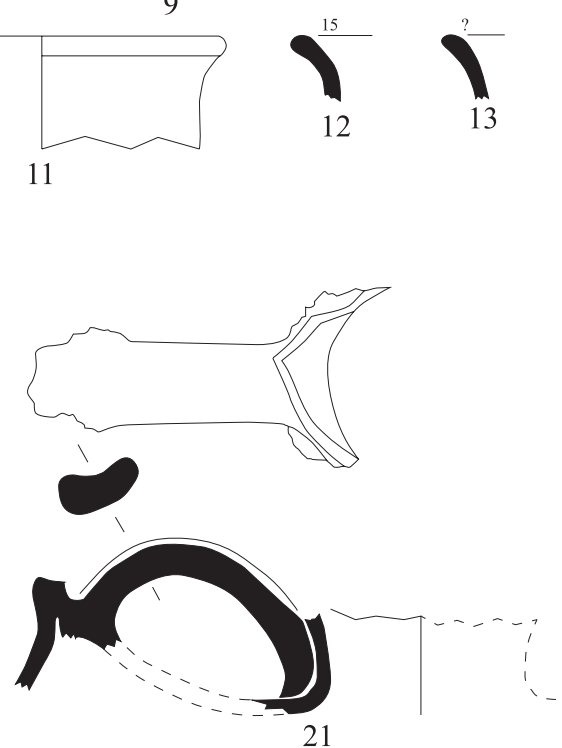

21

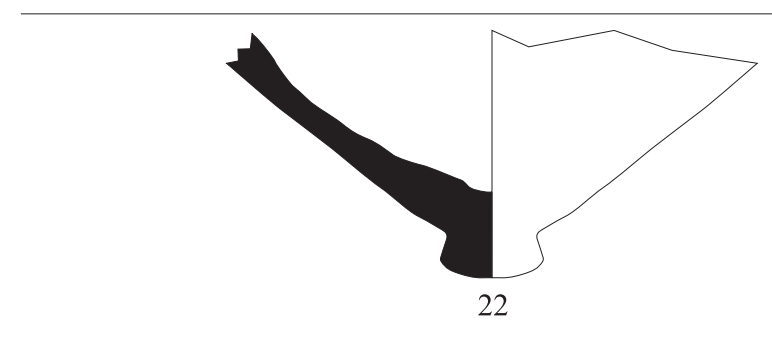

$\mathbf{1}$
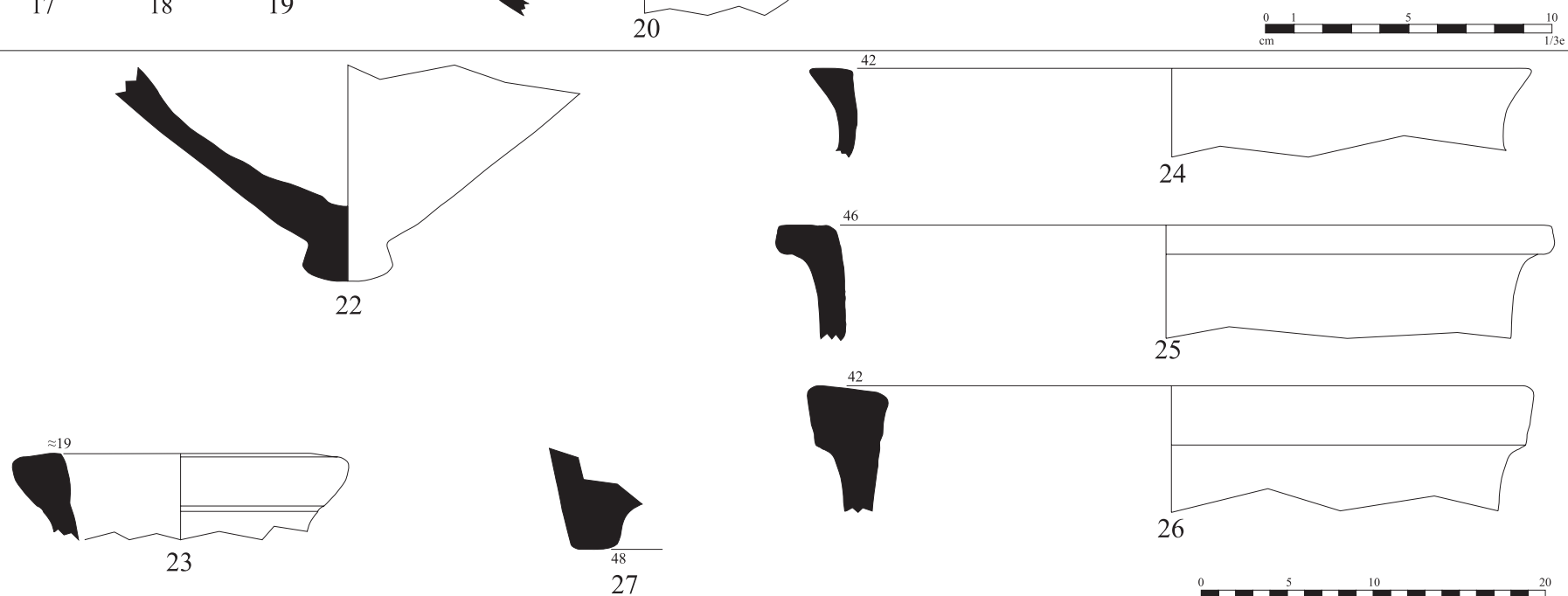

25
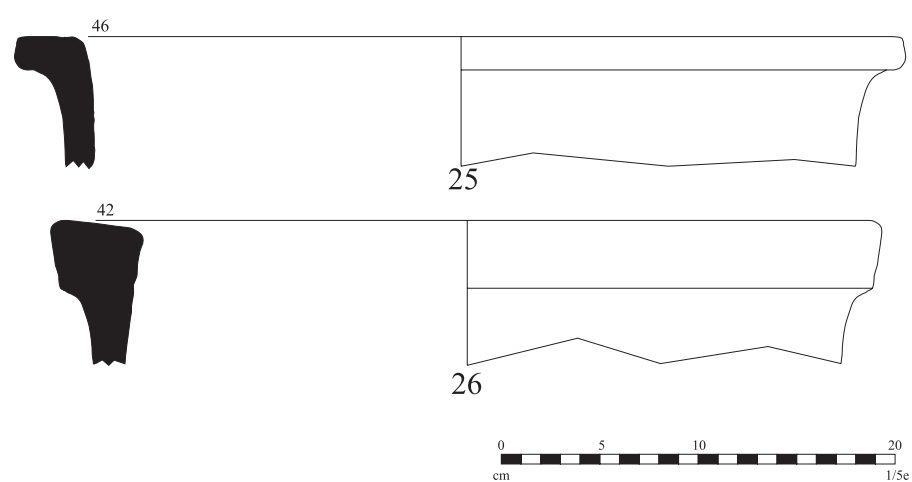

I 31 Mobilier céramique de la période 1 (450-425 av. J.-C.) - nos 1 à 10 : céramique non tournée provençale; nos 11 à 13 : céramique non tournée de l'étang de Berre ; $n^{\circ} 14:$ céramique non tournée à pâte claire ; $n^{\text {os }} 15$ à $19:$ céramique à pâte claire massaliète ; $n^{\text {os }} 20$ et 21 : céramique grise monochrome ; n ${ }^{\circ s} 22$ et 23 : amphore massaliète ; $n^{\circ s} 24$ à 26 : dolium ; $n^{\circ} 27$ : élément en terre crue (A. Cauvin). 


\subsection{La période $2 a$ (425-400 av. J.-C.)}

Cette phase correspond à la reprise de l'occupation primitive, sans qu'aucune structure nouvelle ne vienne modifier les tracés des murs. Cette période est illustrée par 535 fragments et au moins 74 individus (fig. 32).

\subsubsection{Céramique non tournée provençale}

(fig. 33, $n^{o s} 1$ à 27)

À l'instar de la période précédente, les urnes représentent la majorité des formes de la céramique non tournée locale (fig. $33, \mathrm{n}^{\text {os }} 1$ à 24). Les décors, présents sur la panse, jusqu'au contact avec le col, toujours lissé, sont formés de points, d'incisions obliques ou d'un léger bourrelet d'argile imprimé de points (fig. 33, $\mathrm{n}^{\text {os }} 10,23$ et 24). L'urne la mieux conservée est de type CNT-PRO U4 et se distingue

\begin{tabular}{|c|c|c|c|c|c|c|c|c|c|c|c|c|c|}
\hline \multirow{2}{*}{ catégorie } & \multicolumn{3}{|c|}{$\overline{N F R}$} & \multicolumn{3}{|c|}{ NMI } & \multicolumn{3}{|c|}{ NBD } & \multirow{2}{*}{ forme } & \multirow{2}{*}{ type } & \multirow{2}{*}{$\begin{array}{c}\text { éléments } \\
\text { représentés }\end{array}$} & \multirow{2}{*}{ illustrations } \\
\hline & $\mathrm{nb}$ & $\% /$ tot & $\% / g r p$ & $\mathrm{nb}$ & $\% /$ tot & $\% / g r p$ & $\mathrm{nb}$ & $\% /$ tot & $\% / g r p$ & & & & \\
\hline gris mono & 38 & 7,1 & 9,45 & 12 & 16,22 & 17,65 & 10 & 15,63 & 17,24 & $\begin{array}{l}\text { coupe carénée } \\
\text { coupe } \\
\text { oenochoé }\end{array}$ & \begin{tabular}{|l|} 
GR-MONO 3 \\
GR-MONO 5 \\
GR-MONO 8 \\
\end{tabular} & $\begin{array}{l}9 b, 1 t \\
1 b \\
1 f, 1 t \\
\end{array}$ & $\begin{array}{l}\text { fig. } 35, n^{\text {os }} 1-9 \\
\text { fig. } 35, n^{\text {os }} 10-11\end{array}$ \\
\hline cl.-mas & 113 & 21,12 & 28,11 & 16 & 21,62 & 23,53 & 12 & 18,75 & 20,69 & $\begin{array}{l}\text { coupe carénée } \\
\text { coupe à une anse } \\
\text { coupe à une anse } \\
\text { coupe à anses } \\
\text { coupe à anses } \\
\text { cruche } \\
\text { cruche } \\
\text { autre } \\
\text { cruche } \\
\text { olpé }\end{array}$ & $\begin{array}{l}\text { CL-MAS } 222 \\
\text { CL-MAS } 410 \\
\text { CL-MAS } 412 \\
\text { CL-MAS } 425 \\
\text { CL-MAS } 431 \\
\text { CL-MAS } 523 \\
\text { CL-MAS } 541 \\
\text { CL-MAS ind. } \\
\text { CL-MAS ind. } \\
\text { CL-MAS ind. }\end{array}$ & $\begin{array}{l}2 b \\
1 b \\
1 b \\
1 b \\
1 b \\
2 b \\
1 b \\
2 b, 3 a \\
1 f \\
1 b\end{array}$ & $\begin{array}{l}\text { fig. } 34, n^{\circ 5} 5,11 \\
\text { fig. } 34, n^{\circ} 7 \\
\text { fig. } 34, n^{\circ} 6 \\
\text { fig. } 34, n^{\circ} 8 \\
\text { fig. } 34, n^{\circ} 9 \\
\text { fig. } 34, n^{\circ 5} 1-2 \\
\text { fig. } 34, n^{\circ} 3 \\
\text { fig. } 34, n^{\circ} 4 \\
\text { fig. } 34, n^{\circ} 10\end{array}$ \\
\hline pseudo-at & 1 & 0,19 & 0,25 & 1 & 1,35 & 1,47 & 1 & 1,56 & 1,72 & skyphos & $\begin{array}{l}\text { PSEUDO-AT 349- } \\
352\end{array}$ & $1 b$ & fig. $35, n^{\circ} 12$ \\
\hline TOURNÉE FINE & 152 & 28,41 & 37,81 & 29 & 39,19 & 42,65 & 23 & 35,94 & 39,65 & & & & \\
\hline com-mas & 11 & 2,06 & 2,74 & 4 & 5,41 & 5,88 & 4 & 6,25 & 6,9 & $\begin{array}{l}\text { mortier } \\
\text { mortier } \\
\text { mortier } \\
\text { mortier }\end{array}$ & $\begin{array}{l}\text { COM-MAS 621d } \\
\text { COM-MAS 621e } \\
\text { COM-MAS } 631 \\
\text { COM-MAS ind. }\end{array}$ & $\begin{array}{l}1 b \\
1 b \\
1 b \\
1 b\end{array}$ & $\begin{array}{l}\text { fig. } 34, n^{\circ} 14 \\
\text { fig. } 34, n^{\circ} 12 \\
\text { fig. } 34, n^{\circ} 15 \\
\text { fig. } 34, n^{\circ} 13\end{array}$ \\
\hline TOURNÉE COM. & 11 & 2,06 & 2,74 & 4 & 5,41 & 5,88 & 4 & 6,25 & 6,9 & & & & \\
\hline CNT-Pro & 222 & 41,5 & 55,22 & 32 & 43,24 & 47,06 & 29 & 45,31 & 50 & $\begin{array}{l}\text { coupe } \\
\text { coupe } \\
\text { coupelle } \\
\text { urne } \\
\text { autre } \\
\text { jatte } \\
\text { jatte } \\
\text { urne } \\
\end{array}$ & $\begin{array}{l}\text { CNT-PRO C1d } \\
\text { CNT-PRO C2a } \\
\text { CNT-PRO C4 } \\
\text { CNT-PRO ind. } \\
\text { CNT-PRO ind. } \\
\text { CNT-PRO ind. } \\
\text { CNT-PRO J1 } \\
\text { CNT-PRO U4 }\end{array}$ & $\begin{array}{l}1 b \\
1 b \\
1 b \\
9 b, 4 f, 2 d \\
5 b \\
2 b \\
1 b \\
9 b \\
\end{array}$ & $\begin{array}{l}\text { fig. } 33, n^{\circ} 26 \\
\text { fig. } 33, n^{\circ} 25 \\
\text { fig. } 33, n^{\circ} 27 \\
\text { fig. } 33, n^{\circ 5} 3,6,7,14- \\
18,20-24 \\
\text { fig. } 33, n^{\circ 5} 12-13,19 \\
\text { fig. } 33, n^{\circ 5} 1,2,4,5 \text {, } \\
8-11\end{array}$ \\
\hline CNT-Ber & 11 & 2,06 & 2,74 & 2 & 2,7 & 2,94 & 1 & 1,56 & 1,72 & $\begin{array}{l}\text { urne } \\
\text { urne }\end{array}$ & $\begin{array}{l}\text { CNT-BER ind. } \\
\text { CNT-BER U1 }\end{array}$ & $\begin{array}{l}1 f \\
1 b \\
\end{array}$ & fig. $33, n^{\circ} 28$ \\
\hline CNT-Cl & 6 & 1,12 & 1,49 & 1 & 1,35 & 1,47 & 1 & 1,56 & 1,72 & urne & CNT-PRO U5 & $1 b$ & fig. $33, n^{\circ} 29$ \\
\hline NON TOURNÉE & 239 & 44,68 & 59,45 & 35 & 47,29 & 51,47 & 31 & 48,43 & 53,44 & & & & \\
\hline VAISSELLE & 402 & 75,15 & & 68 & 91,89 & & 58 & 90,62 & & & & & \\
\hline a-mas & 103 & 19,25 & 100 & 5 & 6,76 & 100 & 5 & 7,81 & 100 & $\begin{array}{l}\text { amphore } \\
\text { amphore } \\
\text { amphore } \\
\text { amphore }\end{array}$ & $\begin{array}{l}\text { A-MAS bd4 } \\
\text { A-MAS bd5 } \\
\text { A-MAS bd6 } \\
\text { A-MAS ind. }\end{array}$ & $\begin{array}{l}1 b \\
3 b \\
1 b \\
1 f\end{array}$ & $\begin{array}{l}\text { fig. } 35, n^{\circ} 17 \\
\text { fig. } 35, n^{\circ} 13,14,16 \\
\text { fig. } 35, n^{\circ} 15 \\
\text { fig. } 35, n^{\circ} 18\end{array}$ \\
\hline AMPHORES & 103 & 19,25 & & 5 & 6,76 & & 5 & 7,81 & & & & & \\
\hline dolium & 30 & 5,61 & & 1 & 1,35 & & 1 & 1,56 & & $\begin{array}{l}\text { dolium } \\
\text { dolium }\end{array}$ & $\begin{array}{l}\text { DOLIUM bd5 ? } \\
\text { DOLIUM ind. }\end{array}$ & $\begin{array}{l}1 \mathrm{~b} \\
1 \mathrm{~d}\end{array}$ & $\begin{array}{l}\text { fig. } 35, n^{\circ} 19 \\
\text { fig. } 35, n^{\circ} 20\end{array}$ \\
\hline TOTAL & 535 & & & 74 & & & 64 & & & & & & \\
\hline
\end{tabular}

I 32 Période 2a (425-400 av. J.-C.) - Données quantitatives et typologiques du mobilier. 
par son décor de points en haut de la panse et son peignage vertical juste sous le col et devenant oblique au milieu de la panse (fig. 33, $\mathrm{n}^{\circ} 10$ ). Cuite dans une atmosphère non oxydante, sa pâte, très friable, est bien noire ainsi que sa surface, avec des inclusions de chaux et de quartz. Un fragment de décor sort du lot présentant de fines incisions obliques en grain de riz à l'aspect tout à fait différent des autres productions locales. L'intérieur est poli de couleur rouge avec des inclusions de chaux bien visibles. L'extérieur est brun et le peignage est fin (fig. $33, n^{\circ} 23$ ).
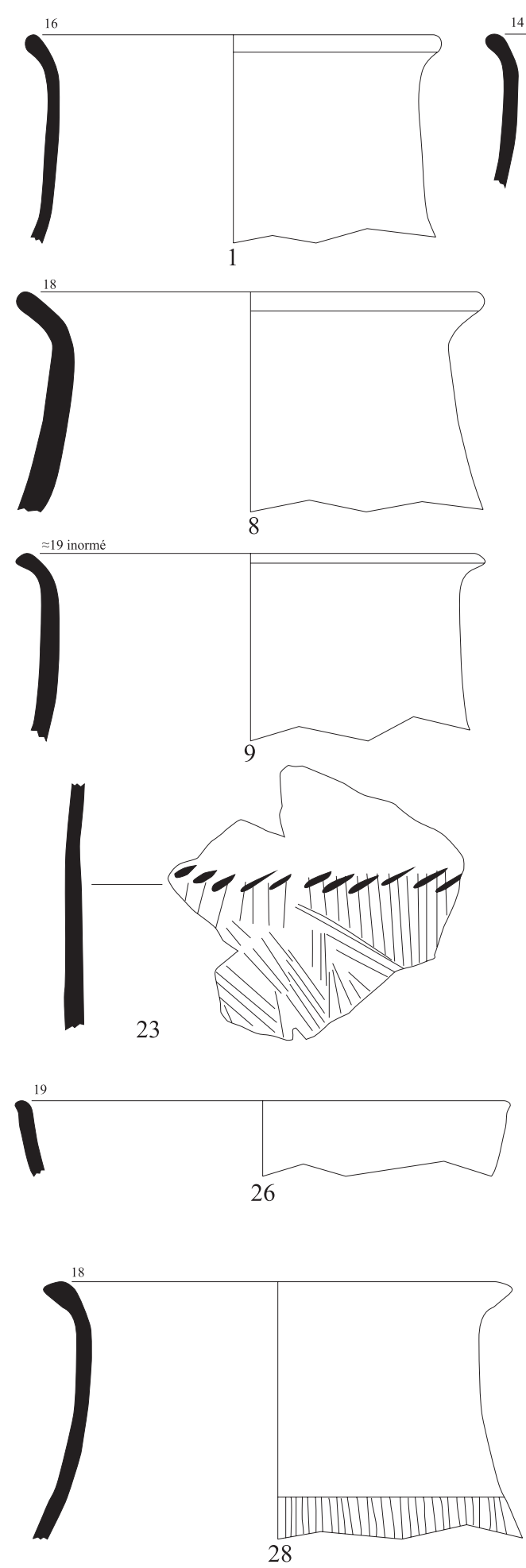
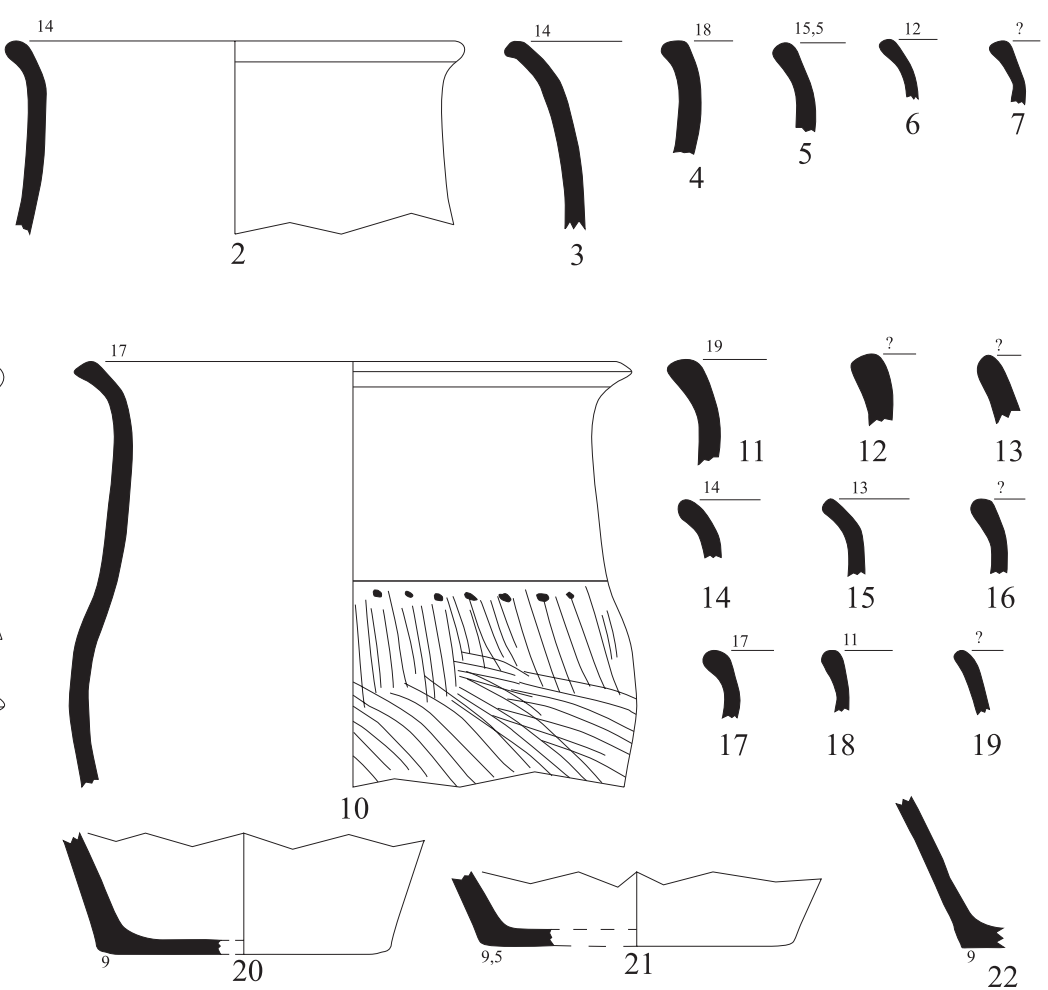

14
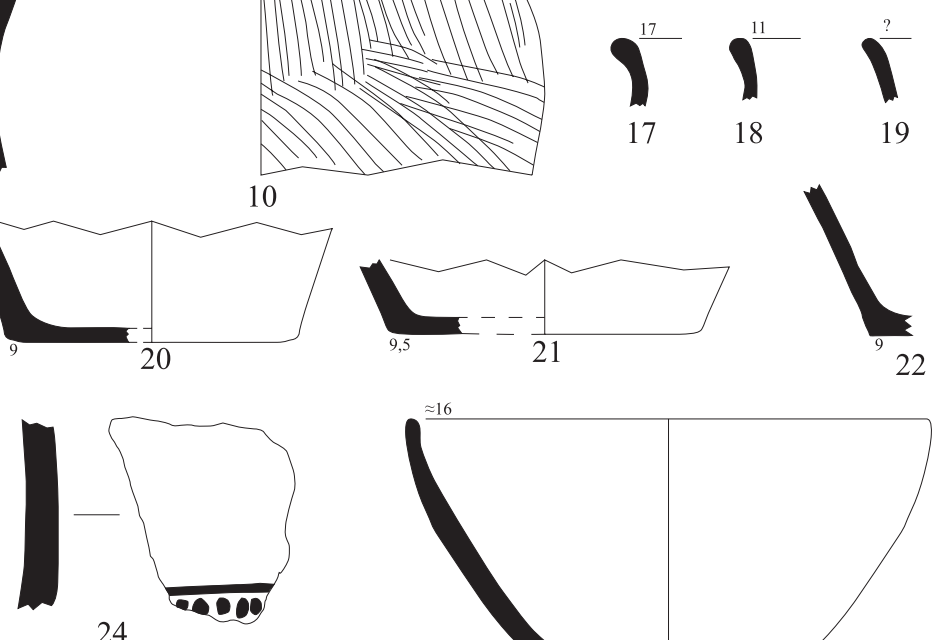

24
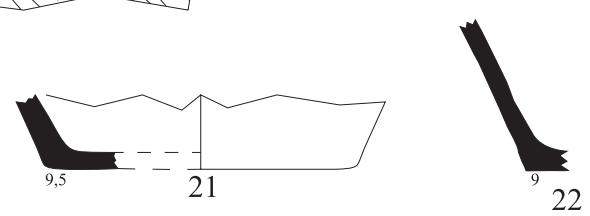

22
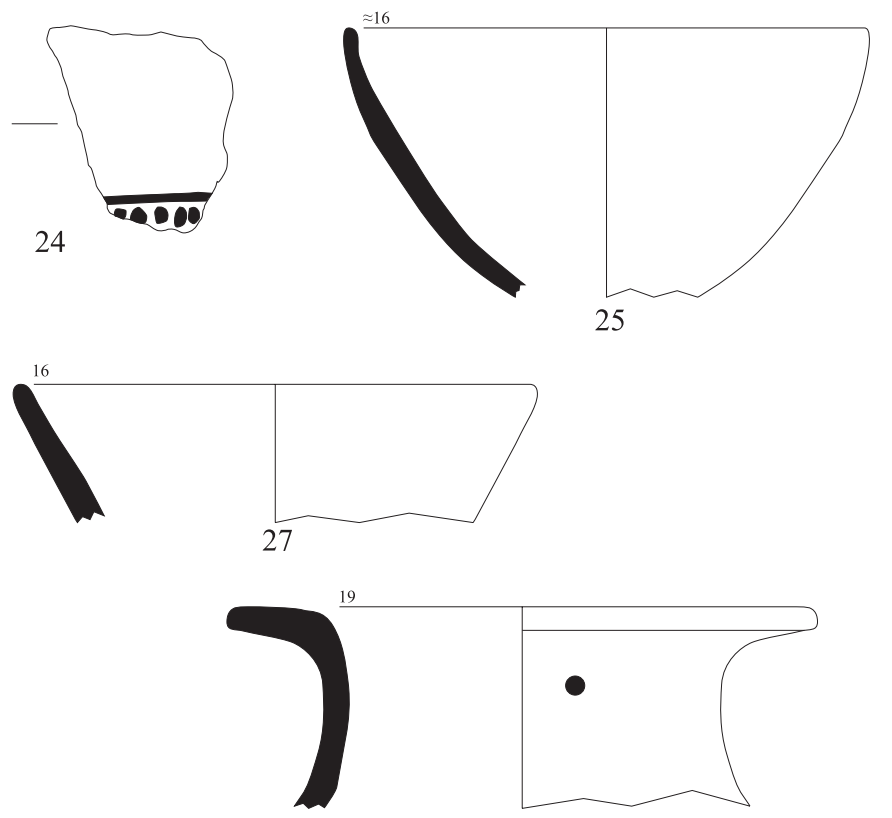

29

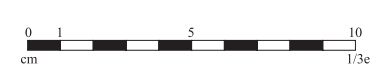

I 33 Mobilier céramique de la période $2 \mathrm{a}(425-400$ av. J.-C.) - nos 1 à 27 : céramique non tournée provençale ; n 28 : céramique non tournée de l'étang de Berre; $n^{\circ} 29$ : céramique non tournée à pâte claire (A. Cauvin). 
Les coupes sont plus nombreuses avec des formes très variables. L'une, de type CNT-PRO C2a, à la vasque profonde présente des parois divergentes et des bords relevés (fig. 33, $\mathrm{n}^{\circ} 25$ ). Sur le site de Tamaris, une coupe similaire est attestée et, selon S. Duval, le léger biseau de la lèvre pourrait prouver une utilisation comme couvercle (Duval 1998). Une deuxième coupe, de type CNT-PRO Cld, présente des flancs divergents avec léger déversement de la lèvre vers l'extérieur. Elle est extrêmement bien lissée et sa pâte présente très peu d'inclusions. Par sa finition remarquable, il s'agit peut-être d'une production des ateliers de l'étang de Berre (fig. 33, $\mathrm{n}^{\circ}$ 26). La troisième a la forme d'une écuelle de type CNT-PRO C4, avec un bord rectiligne divergent et un lissage bien réalisé (fig. 33, n² 27).

\subsubsection{Céramique non tournée des rives de l'étang de} Berre (fig. 33, $n^{\circ} 28$ )

Une forme d'urne est attestée et présente une pâte grise homogène sonnante, au dégraissant visible. Son polissage et son peignage vertical régulier sont finement exécutés, et elle présente un bord déversé assez anguleux presque triangulaire.

\subsubsection{Céramique non tournée à pâte claire}

(fig. 33, $n^{\circ}$ 29)

L'urne CNT-CL U5, qui ressemble à l'exemplaire de la période 1 , présente une pâte identique. Les différentes irrégularités de sa face interne trahissent la technique du colombin (fig. 33, n²9). Elle est percée d'un trou effectué après cuisson parfaitement régulier quelques centimètres sous la lèvre.

\subsubsection{Céramique à pâte claire massaliète \\ (fig. 34, $n^{o s} 1$ à 10)}

Bien mieux représentée que durant la période précédente, la céramique à pâte claire massaliète est également plus variée. Les formes sont toujours liées au service et à la consommation des aliments avec des cruches (fig. $34, \mathrm{n}^{\text {os }} 1$ à 3 et 10) et des coupes individuelles (fig. $34, n^{\text {os }} 5$ à 9).

Deux types de cruches sont attestées: le type CL-MAS 523 avec une lèvre arrondie dans le prolongement du col (fig. $34, \mathrm{n}^{\text {os }} 1$ et 2). Une cruche de type CL-MAS 541 est bien conservée. Elle se distingue par une absence de col, une lèvre anguleuse et des sillons sur le haut de sa panse (fig. $34, n^{\circ} 3$ ). Notons également la présence d'une cruche à fond annulaire dont la panse est peinte de deux bandes marron (fig. $34, n^{\circ} 10$ ).

Une plus grande variété pour les coupes existe: elles sont peintes d'un engobe rouge plus ou moins bien conservé.
Une coupe carénée CL-MAS 222 a conservé des traces orangées à l'intérieur et à l'extérieur et présente une rainure soulignant sa carène (fig. $34, n^{\circ} 5$ ). Sur une coupe CL-MAS 412, peinte à l'intérieur, on distingue encore plusieurs nuances de couleur allant de l'orange au brun (fig. $34, n^{\circ} 6$ ). Sa lèvre est légèrement biseautée vers l'intérieur. Sur une coupe de la série CL-MAS 410 on retrouve le même type de peinture (fig. $34, n^{\circ} 7$ ). Sa lèvre est bien épaissie par rapport aux restes de la paroi et l'inflexion du milieu de panse est assez marquée permettant de la rapprocher de la variante CL-MAS $412 \mathrm{~b}$. Une coupe de type CL-MAS 425 se distingue par sa peinture interne aux bandes de différentes couleurs très bien conservées (fig. $34, n^{\circ} 8$ ). On peut également noter la présence de petits sillons à l'extérieur juste sous la lèvre. Signalons également la présence d'une coupe à anses CL-MAS 431 aux fines parois peintes présentant trois petits sillons sous la lèvre (fig. $34, n^{\circ} 9$ ).

Mais l'élément le plus remarquable est un bord de coupe carénée à la pâte épurée avec un engobe rouge très bien conservé qui présente des traces de plusieurs sillons à l'instar des décors ondés des céramiques grises monochromes (fig. 31, $\left.\mathrm{n}^{\circ} 11\right)$. Ces coupes parfois à décor ondé sont, semble-t-il, attestées sur le site de l'Île ainsi qu'à Saint-Pierre-les-Martigues (rens. J. Chausserie-Laprée).

\subsubsection{Céramique commune massaliète}

(fig. 34, $n^{o s} 12$ à 15 )

Cette catégorie est illustrée dans l'Île uniquement par des formes de mortiers utilisant la même argile que les amphores.

Deux types de mortier sont attestés. Le premier, de type COM-MAS 621e, présente une vasque profonde avec une lèvre arrondie non pendante (fig. $34, n^{\circ} 12$ ). Le second, très fragmentaire, a une lèvre assez épaisse avec un petit sillon sous la lèvre (fig. $34, \mathrm{n}^{\circ}$ 13). Le mortier COM-MAS 623c, à la vasque profonde et au diamètre important, caractérisé par ses deux petits sillons sous la lèvre présente un bord au bourrelet arrondi (fig. 34, n ${ }^{\circ} 14$ ). L'élément le plus notable est un mortier à vasque profonde à la pâte blanche, finement dégraissée, présentant un marli horizontal de type COM-MAS 631. Celui-ci est décoré d'une incision ondée courant sur tout le pourtour du vase (fig. $34, n^{\circ} 15$ ).

\subsubsection{Céramique pseudo-attique (fig. $35, n^{\circ} 12$ )}

Déjà publié en 1995 (Chausserie-Laprée, 1995, fig. 2, nº 4), un bord d'une coupe-skyphos avait été identifiée comme une production étrusque. Il s'agit en réalité d'un skyphos pseudo-attique de type PSEUDO-AT 349-352 imitant les skyphos attiques aux décors de rehauts blancs en guir- 
lande de type AT-FR Sk2e ${ }^{17}$. Il se caractérise par sa pâte jaune couverte d'une fine peinture fragile.

\subsubsection{Amphores et vases de stockage}

(fig. 35, $n^{o s} 13$ à 20)
Parmi les fragments d'amphores massaliètes, on peut noter la présence de plusieurs bords de type bd5. Une amphore est représentée par une partie de son col, de son anse et de sa panse (fig. $35, n^{\circ} 13$ ). Sur le col sont gravés après la cuisson sept traits verticaux légèrement obliques comme on en
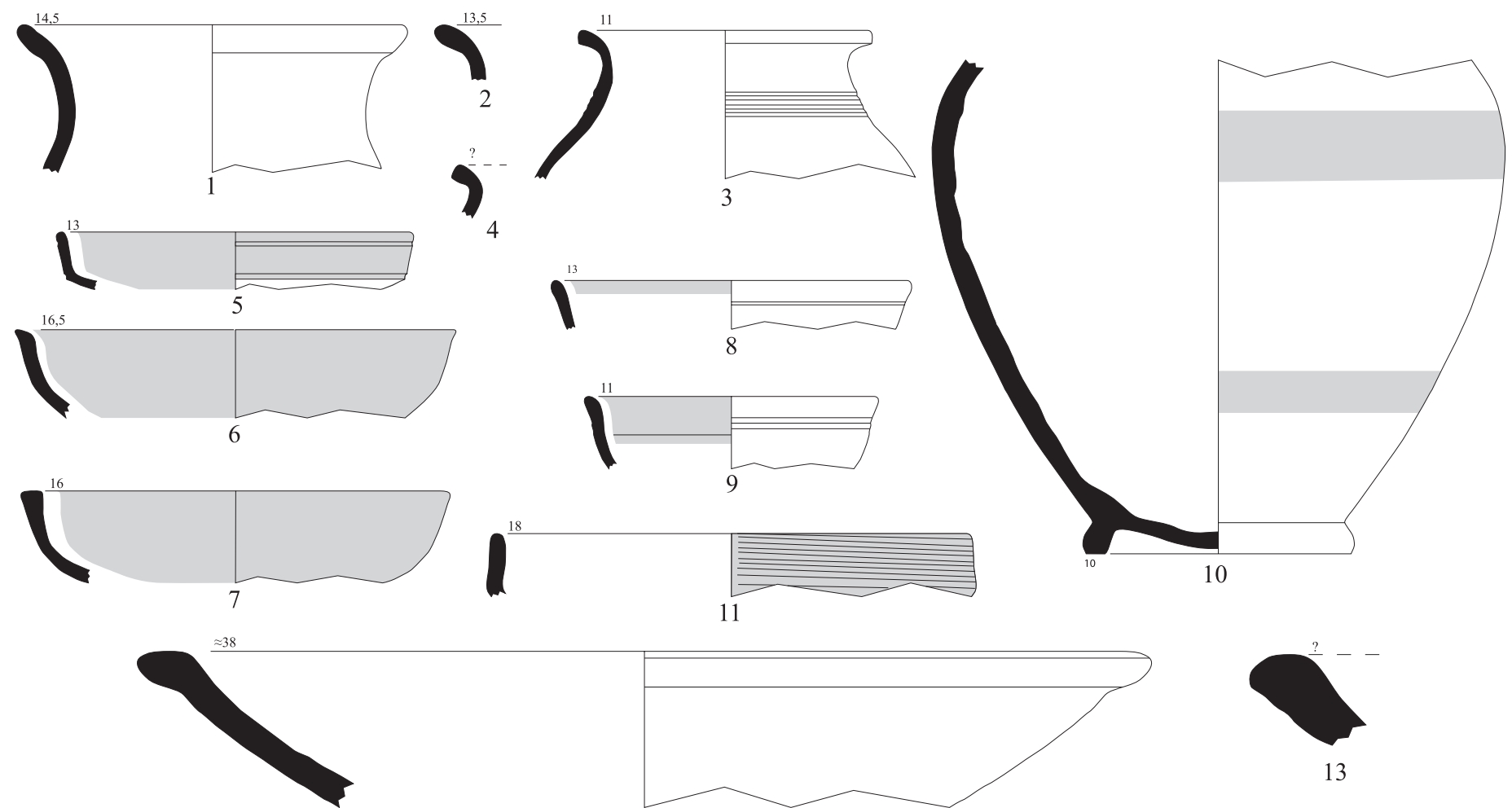

11
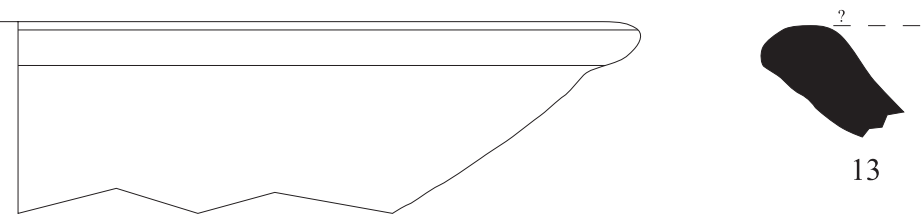

13

12
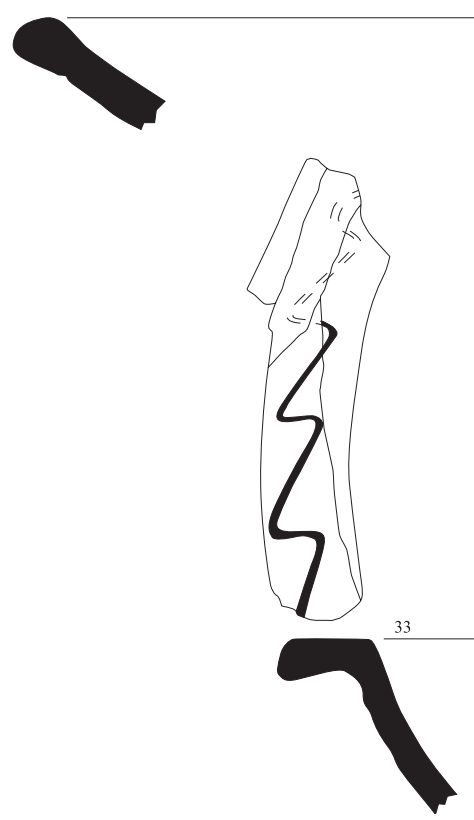

14
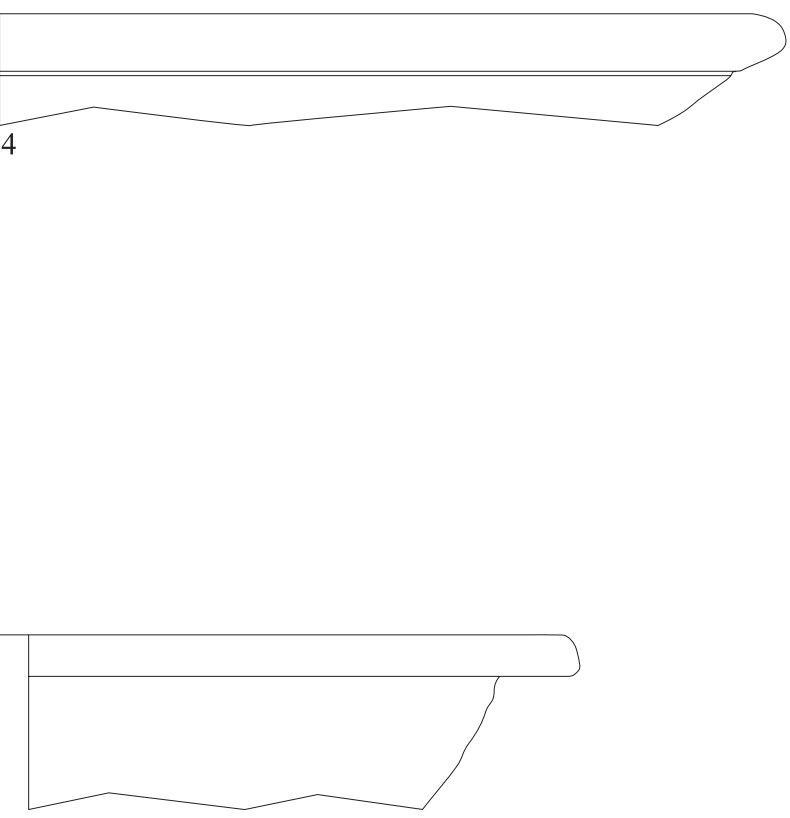

15

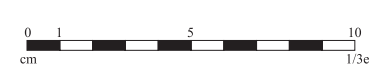

I 34 Mobilier céramique de la période $2 \mathrm{a}\left(425-400\right.$ av. J.-C. $-\mathrm{n}^{\circ \mathrm{s}} 1$ à 10 : céramique à pâte claire massaliète ; $\mathrm{n}^{\circ} 11$ : céramique à pâte claire locale; $\mathrm{n}^{\text {os }} 12$ à 15 : mortier massaliète. 

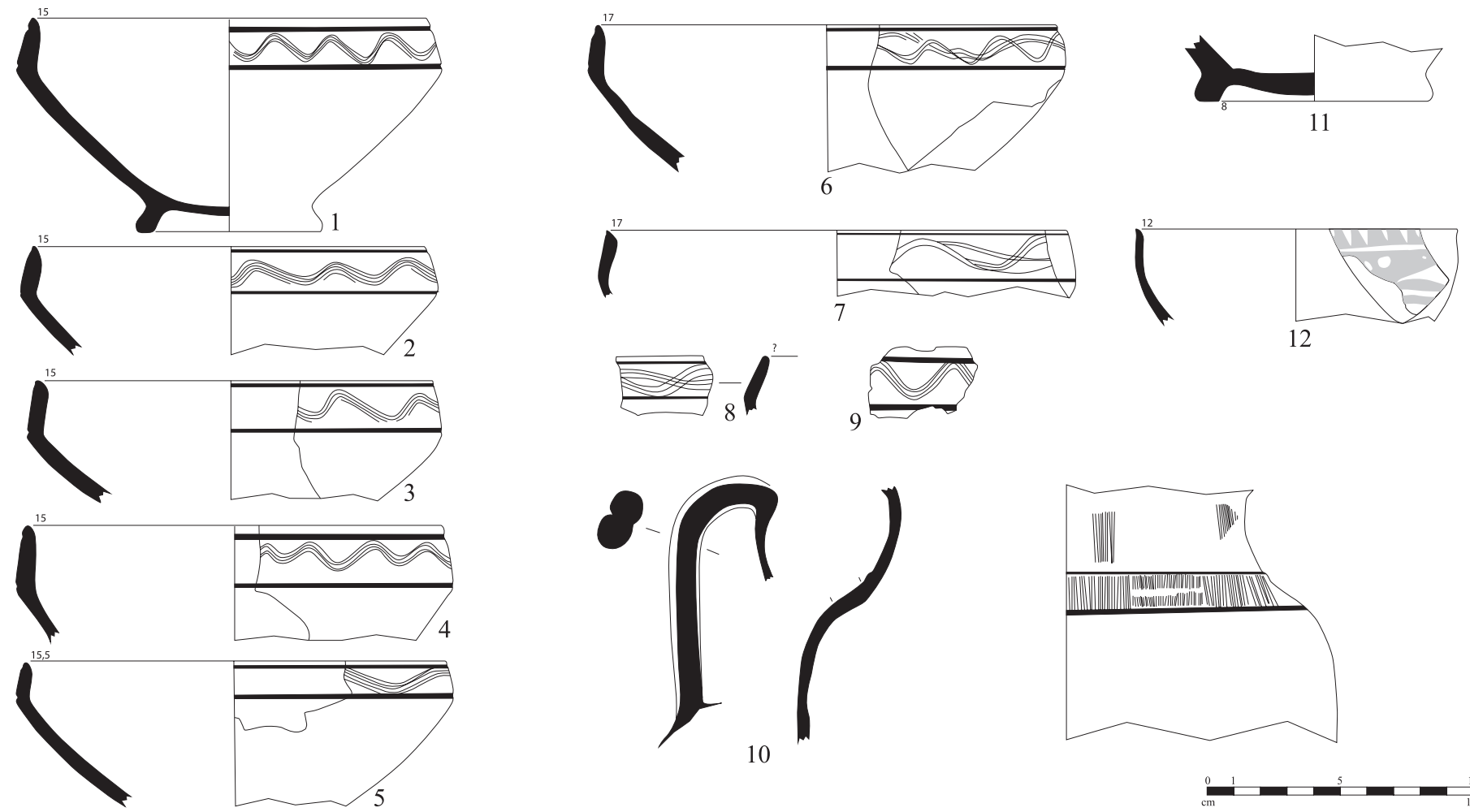

10

12

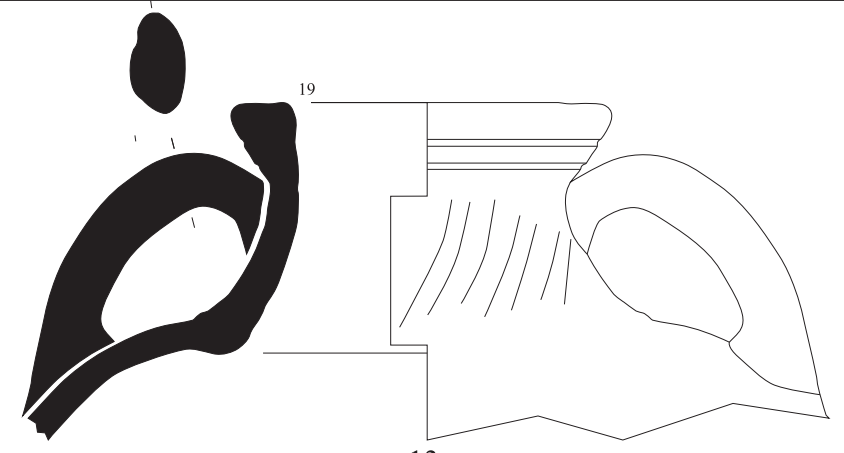

13

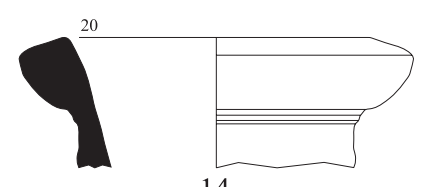

14

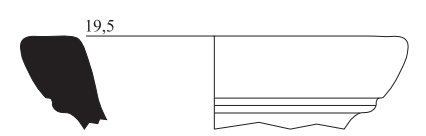

15

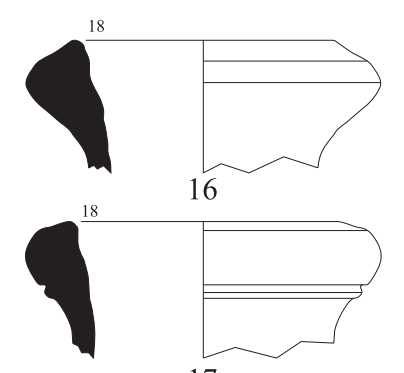

17
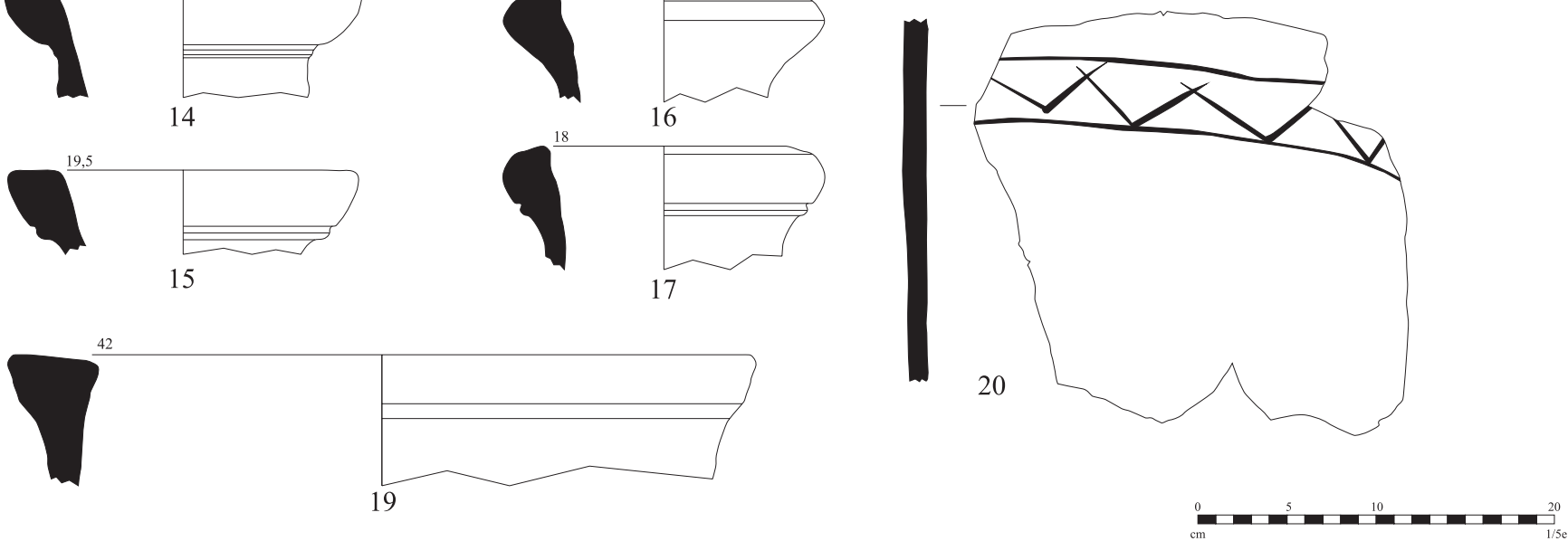

I 35 Mobilier céramique de la période $2 \mathrm{a}$ (425-400 av. J.-C.) - $\mathrm{n}^{\text {os }} 1$ à 11 : céramique grise monochrome ; $n^{\circ} 12$ : céramique pseudo-attique; nos 13 à 18 : amphore massaliète ; nos 19 et 20 : dolium

(A. Cauvin). 
retrouve parfois sur ces amphores (Py et al. 2001, $\mathrm{n}^{\text {os }} 1275$, 1276, p. 239). Ces gravures post-cuisson sont aussi attestées dans d'autres îlots du site (Chausserie-Laprée 2004, fig. $55, \mathrm{n}^{\text {os }} 5$ et 6 ). Leur signification demeure inconnue même si l'on leur attribue parfois un rôle (de décompte?) dans les pratiques commerciales. Un bord d'amphore massaliète de type A-MAS bd4 a un sillon très marqué, avec des facettes émoussées (fig. 35, $\mathrm{n}^{\circ}$ 17).

Plusieurs morceaux de doliums, à la pâte grise et à la couverte rosée, présentent un décor de chevrons assez finement incisés sur la panse (fig. $35, n^{\circ} 20$ ). Si l'épaisseur peu importante de ce vase l'assimile davantage à la catégorie des urnes, sa pâte grossière ne permet pas de la ranger dans la catégorie des CNT-PRO. Un bord de dolium de type bd5 est quant à lui très massif et sa pâte est très grossière, avec un dégraissant important et des inclusions de chamotte (fig. $\left.35, \mathrm{n}^{\circ} 19\right)$.

\subsection{Période $2 b$ (400-375/360 av. J.-C.)}

Cette phase correspond à la mise en place du découpage de l'îlot $\mathrm{H}$ en différentes maisons. Le rempart semble aussi avoir été reconstruit durant cette période. Le mobilier céramique étudié compte 848 fragments et 75 individus (fig. 36).

\subsubsection{Céramique non tournée provençale \\ (fig. 37, $n^{\text {os }} 1$ à 30)}

La céramique non tournée provençale est représentée majoritairement par des urnes, au sein desquelles on relève de nouvelles formes (fig. 37, $\mathrm{n}^{\text {os }} 1$ à 20 ), notamment les urnes de type CNT-PRO U5 avec leur lèvre très déversée (fig. $37, \mathrm{n}^{\text {os }} 13$ et 14). L'une d'entre elles, cuite en atmosphère oxydante, présente une teinte rosée avec une pâte criblée d'inclusions de calcite à la manière des doliums (fig. $37, \mathrm{n}^{\circ} 13$ ). Notons également une urne de type CNT-PRO U3b aux parois rugueuses (fig. 37, $\mathrm{n}^{\circ}$ ).

Les premières imitations de céramique non tournée de l'étang de Berre semblent faire leur apparition illustrée par une urne de type CNT-PRO U4 bien cuite, mais au dégraissant abondant et grossier (fig. 37, $\mathrm{n}^{\circ} 1$ ). Un élément de décor avec de fines incisions obliques est aussi à noter (fig. $37 \mathrm{n}^{\circ} 12$ ).

Les jattes, qui font leur apparition, sont toutes de type CNTPRO Jla avec un décor sur la lèvre plus ou moins régulier (fig. 37, $\mathrm{n}^{\text {os }} 23$ à 26 et 28, 29). De diamètre très varié (entre 19 et $30 \mathrm{~cm}$ ), elles possèdent des lèvres de formes différentes, tantôt élargies avec un décor plus imposant, tantôt plus anguleuses ou arrondies.

Des bords de coupes sont également notables (fig. $37, \mathrm{n}^{\text {os }} 27$ et 30). L'une, de type CNT-PRO C1, a une pâte orange clair avec des inclusions de calcite (fig. $37, \mathrm{n}^{\circ} 27$ ).

Les mortiers en céramique non tournée provençale constituent la nouveauté de cette période. Ils se caractérisent par leur épaisseur et leur important dégraissant (fig. 37, $\mathrm{n}^{\text {os }} 21$ et 22). Un exemplaire complet est attesté durant cette période dans l'îlot A (Damotte 2003, fig. 20, n 30).

\subsubsection{Céramique non tournée des rives de l'étang de Berre (fig. 37, $n^{\text {os }} 31$ à 37)}

La période $2 \mathrm{~b}$ est celle durant laquelle la CNT-BER est la plus commune: elle est illustrée par quelques urnes. Un fragment particulier, qui présente un peignage très régulier (fig. 37, $\mathrm{n}^{\circ} 37$ ), est représentatif du soin apporté au décor, ici un quadrillage. Cette grande qualité d'exécution et de finition, propre à cette production, a déjà été signalée (Chausserie-Laprée, Nin 1988, 84, fig. 2 et 3 ; Damotte 2003, fig. 21).

\subsubsection{Céramique non tournée à pâte claire}

(fig. 37, $n^{\circ} 38$ )

Une coupe à pâte claire de type Cla, aux parois divergentes rectilignes, est identifiée. Sa pâte est très proche de celle des productions massaliètes, mais avec un dégraissant plus important (fig. 37, $\mathrm{n}^{\circ} 38$ ).

\subsubsection{Céramique à pâte claire massaliète (fig. 38, $n^{o s} 1$ à 17)}

Bien plus abondante que dans les niveaux précédents, cette production est également plus variée dans son répertoire.

On retrouve des cruches (fig. $38, \mathrm{n}^{\text {os }} 1$ à 5 ), des coupes (fig. $38, \mathrm{n}^{\text {os }} 6$ à 11) et d'autres formes incertaines ou anecdotiques (fig. 38, $\mathrm{n}^{\text {os }} 12$ à 14). Les cruches sont, soit du type CL-MAS 542 à lèvre anguleuse (fig. $38, n^{\text {os }} 1$ et 2 ) ou plus arrondie (fig. $38, \mathrm{n}^{\text {os }} 3$ et 4), soit du type CL-MAS 525 avec une lèvre repliée à l'oblique. Les coupes CL-MAS 410 sont toutes peintes (fig. 38, $\mathrm{n}^{\text {os }} 6$ à 9). Deux bords de type CL-MAS 425 sont également conservés (fig. $38, \mathrm{n}^{\text {os }} 10$ et 11). Parmi les fonds (fig. $38, n^{\text {os }} 15$ à 17 ), on note un fond annulaire à pâte orange assez bas et peint à la base d'une peinture orange rouille (fig. $38, \mathrm{n}^{\circ} 15$ ).

Un fragment de pied, aux arêtes vives et nettes, d'une céramique à pâte assez fine et grise, peut-être de la grisemonochrome, est aussi attesté (fig. 38, no 18). 


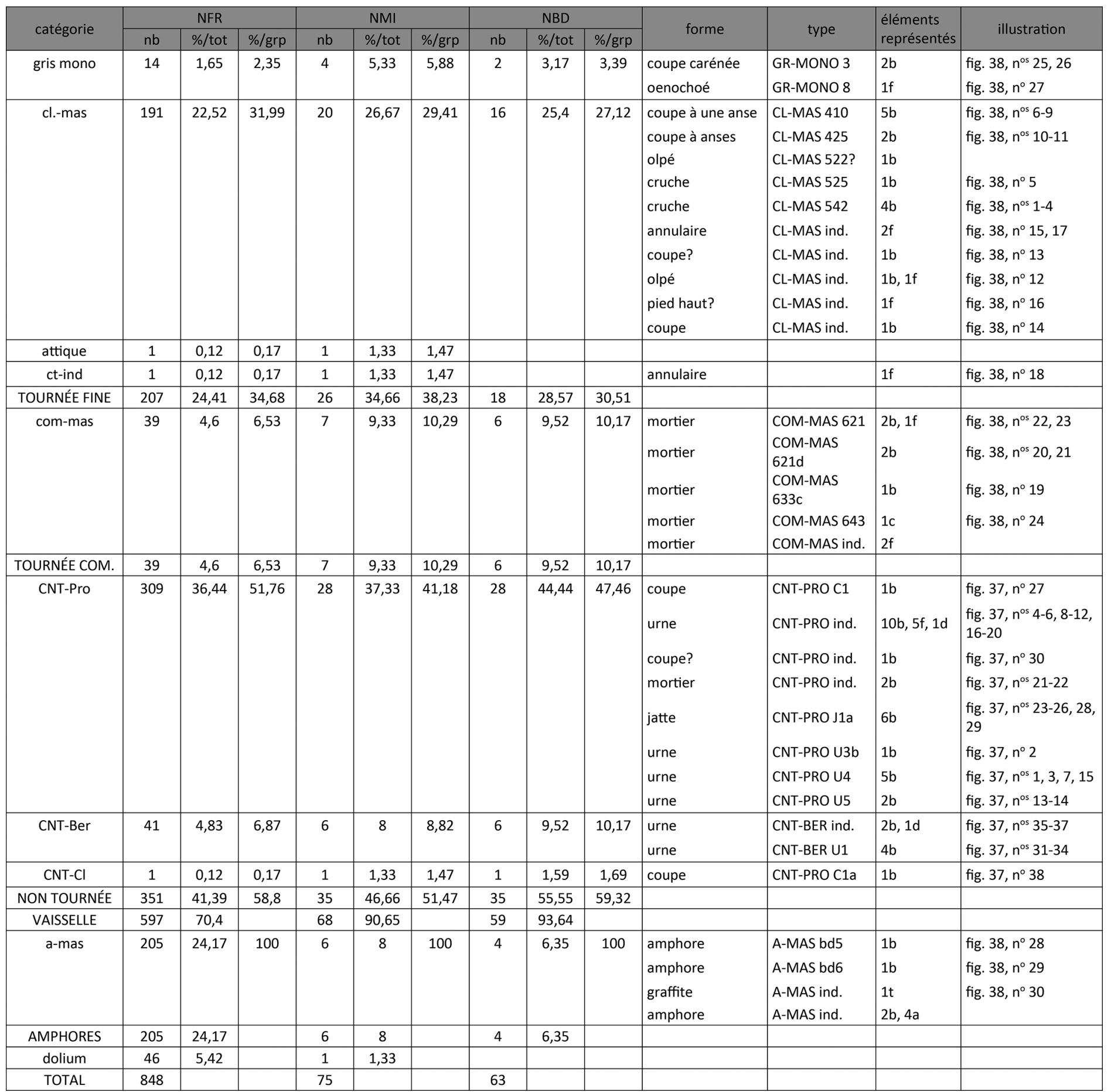

I 36 Période 2b (400-375 av. J.-C.) - Données quantitatives et typologiques du mobilier. 

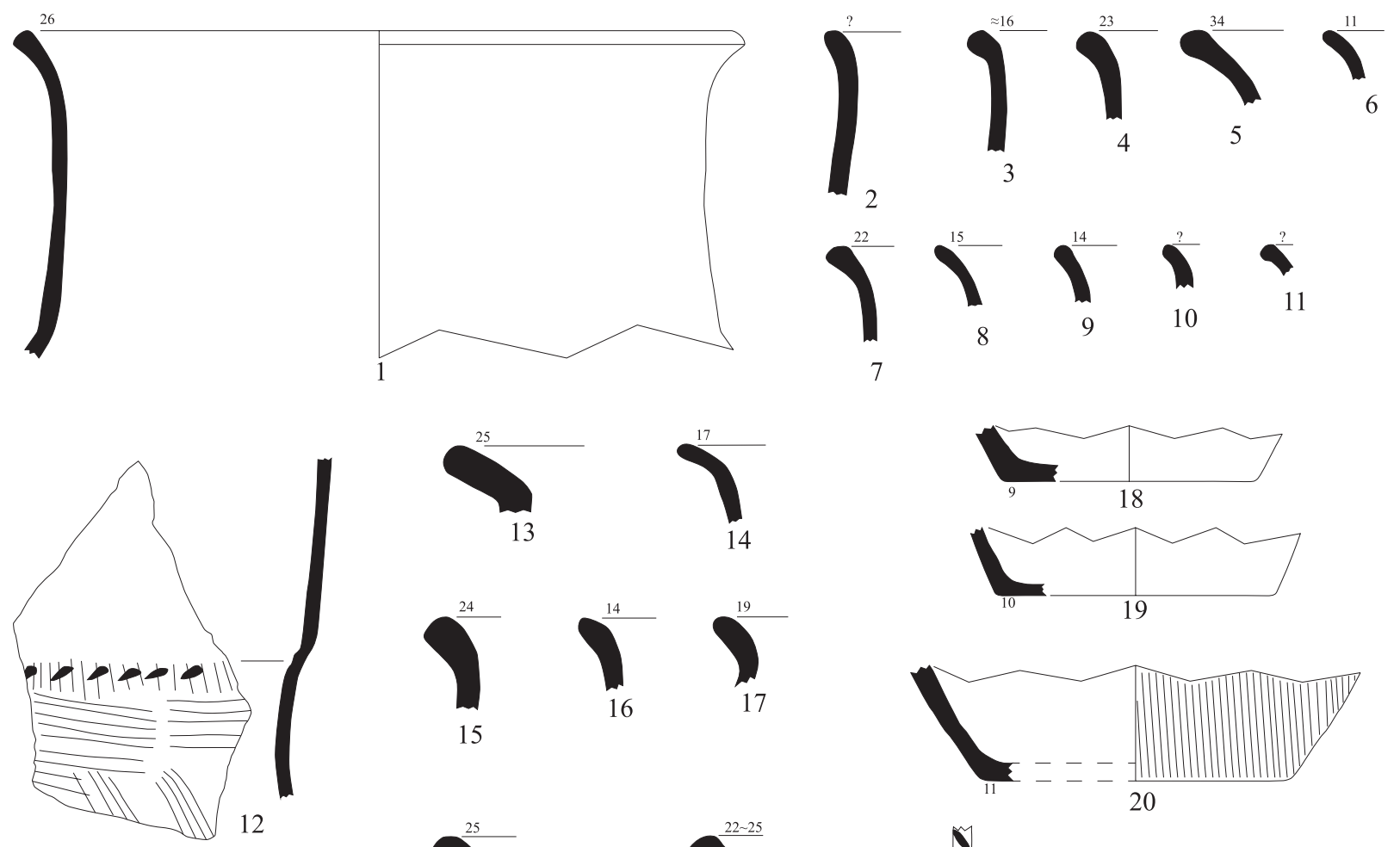

13

14
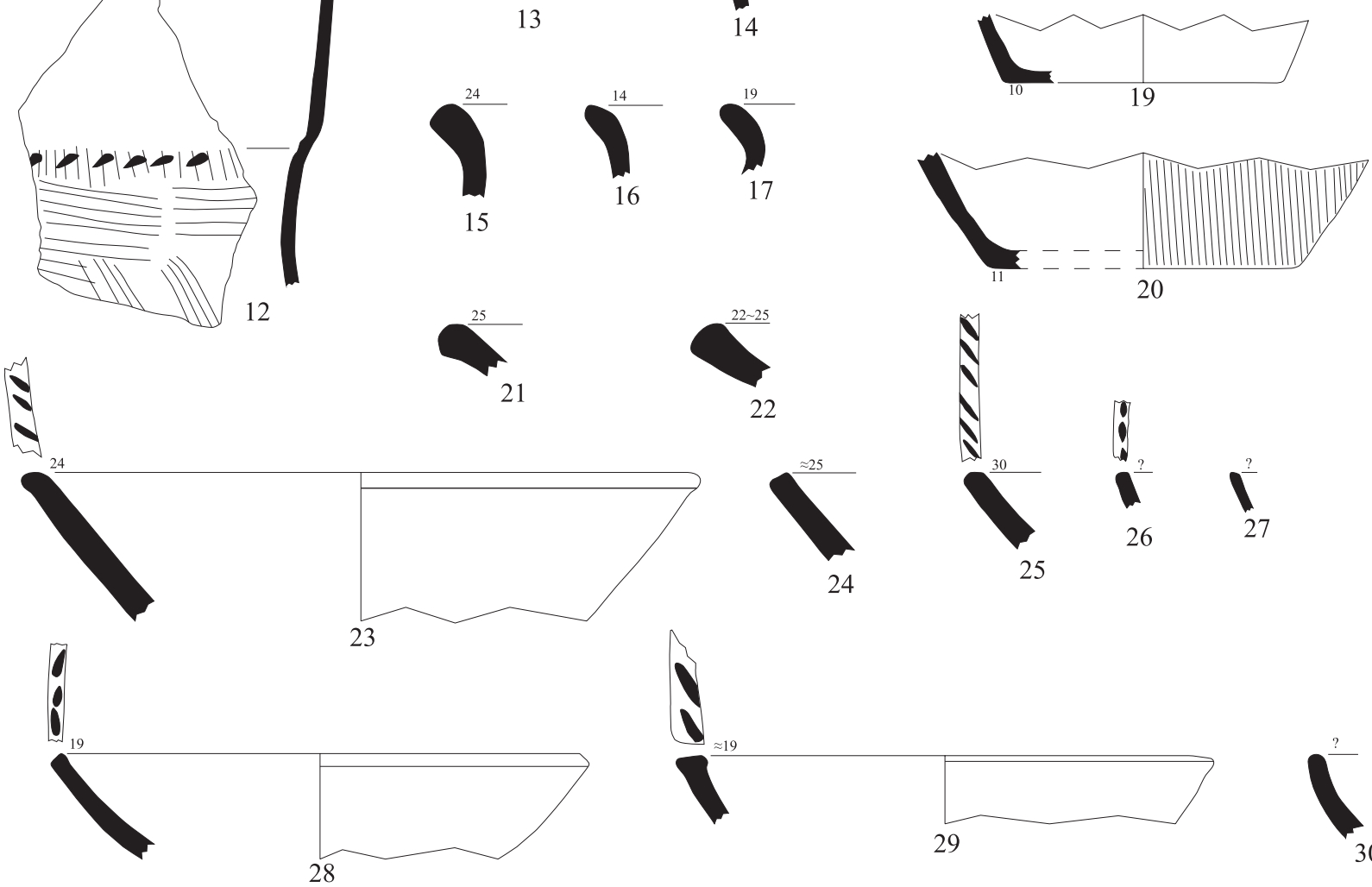

24
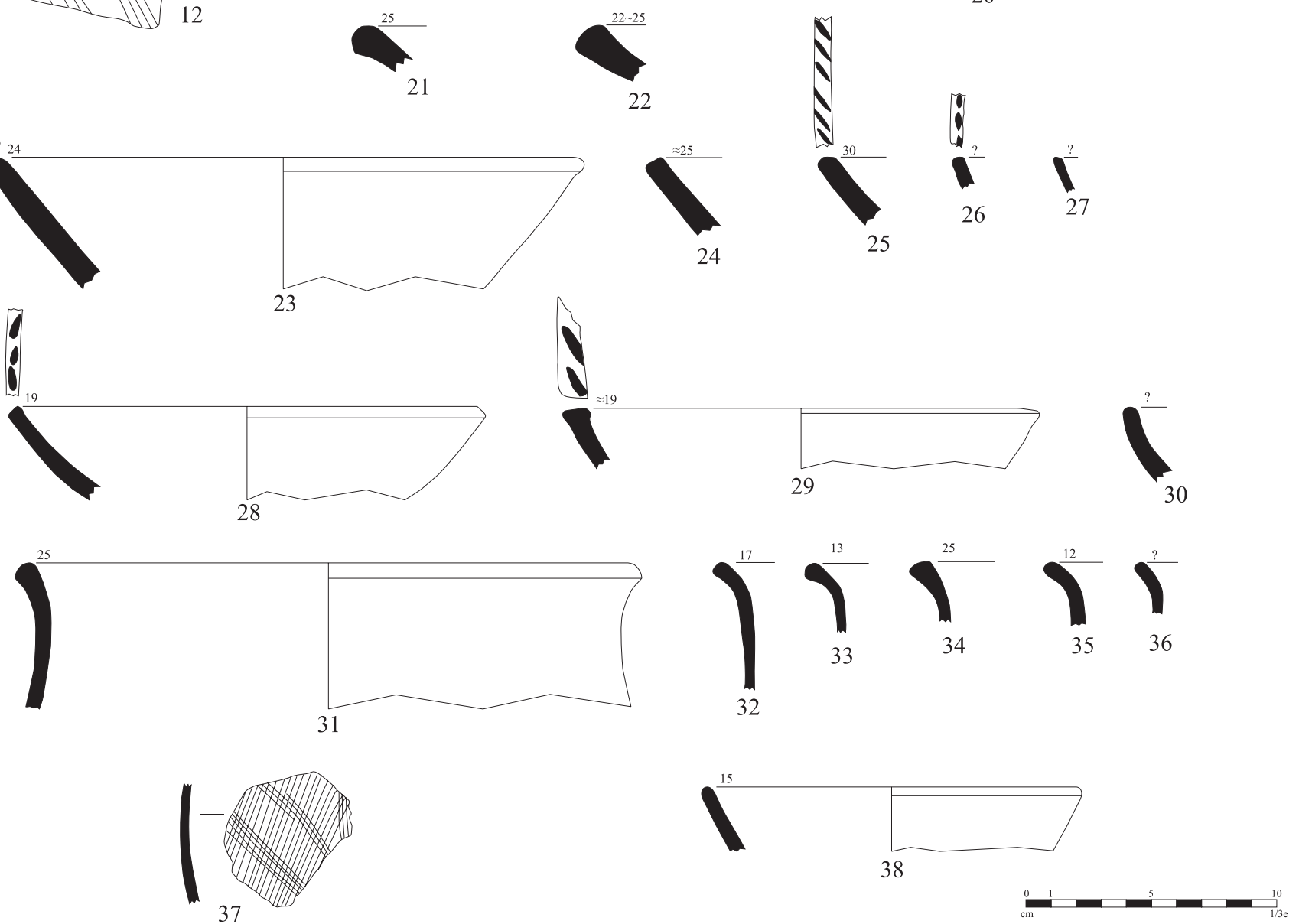

I 37 Mobilier céramique de la période $2 \mathrm{~b}\left(400-375\right.$ av. J.-C.) - $\mathrm{n}^{\text {os }} 1$ à 30 : céramique non tournée provençale ; nos 31 à 37 : céramique non tournée de l'étang de Berre ;

$\mathrm{n}^{\circ} 38$ : céramique non tournée à pâte claire

(A. Cauvin). 

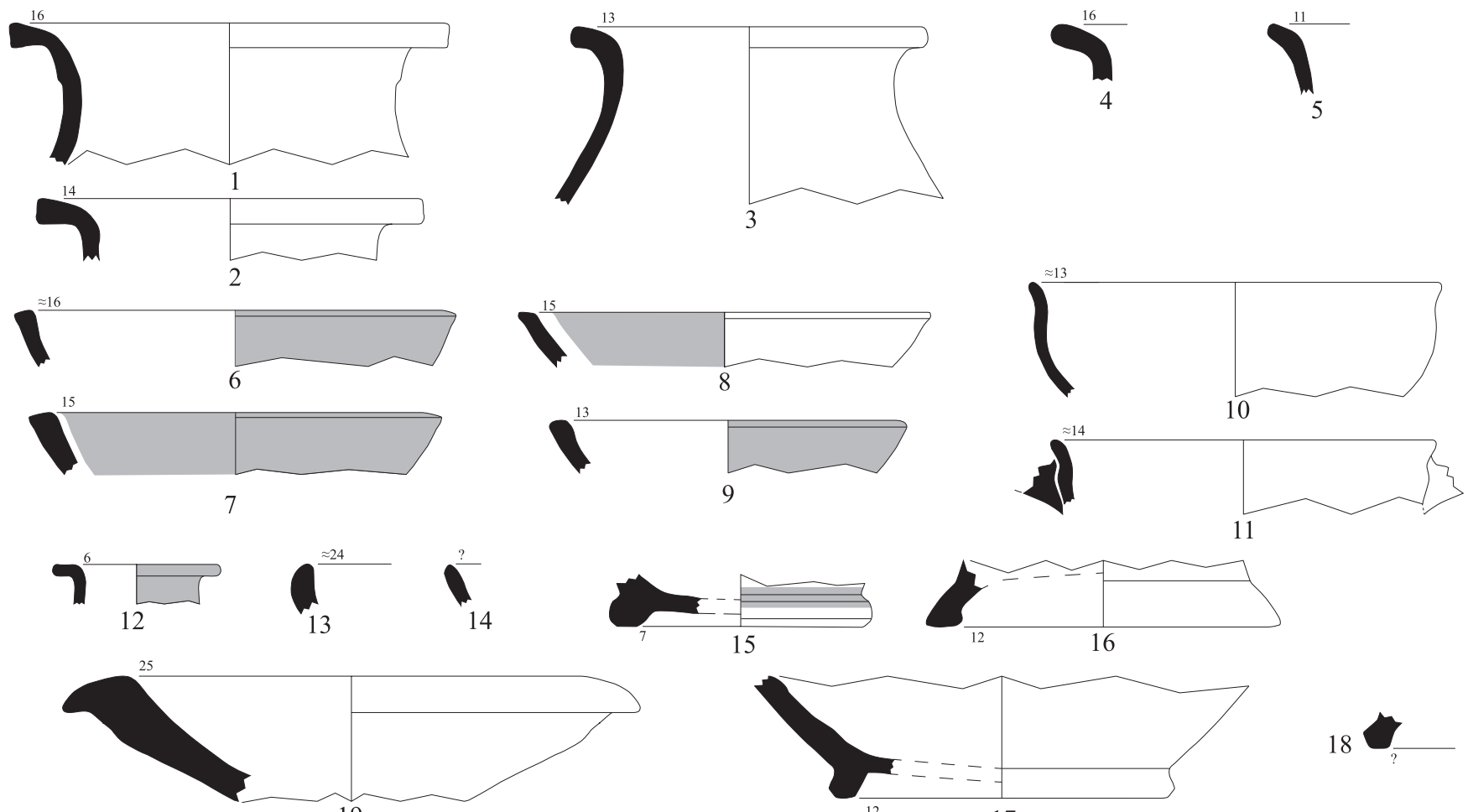

19
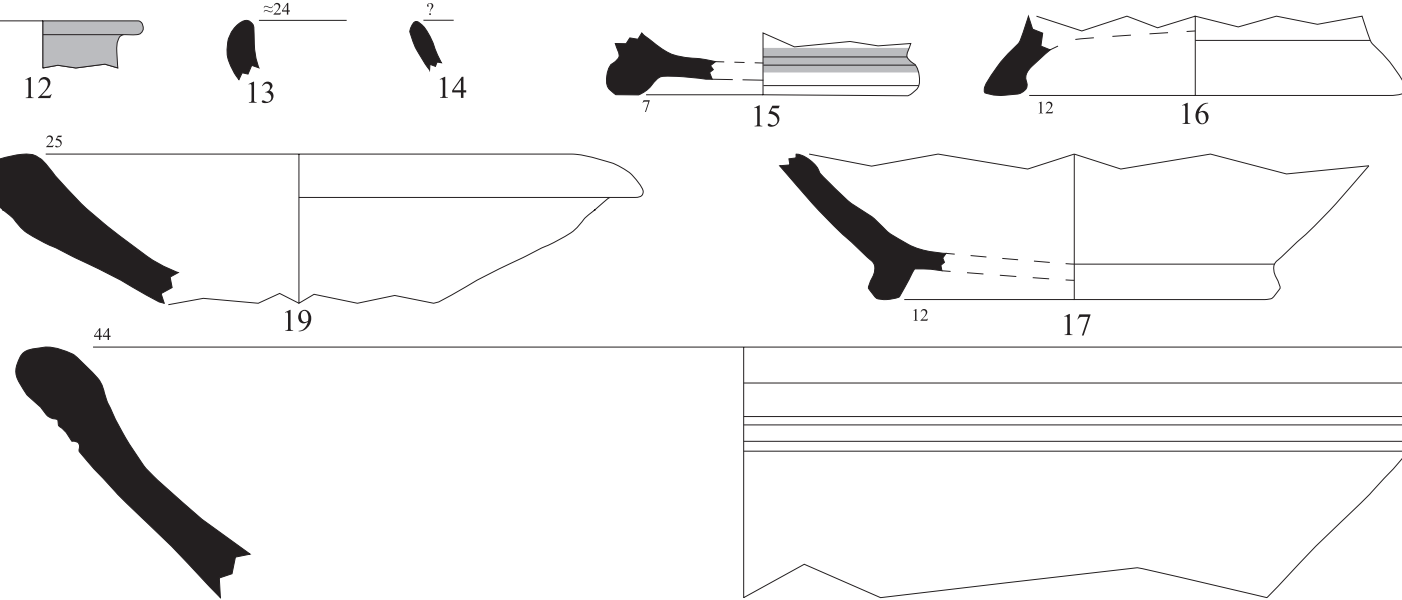

18

17
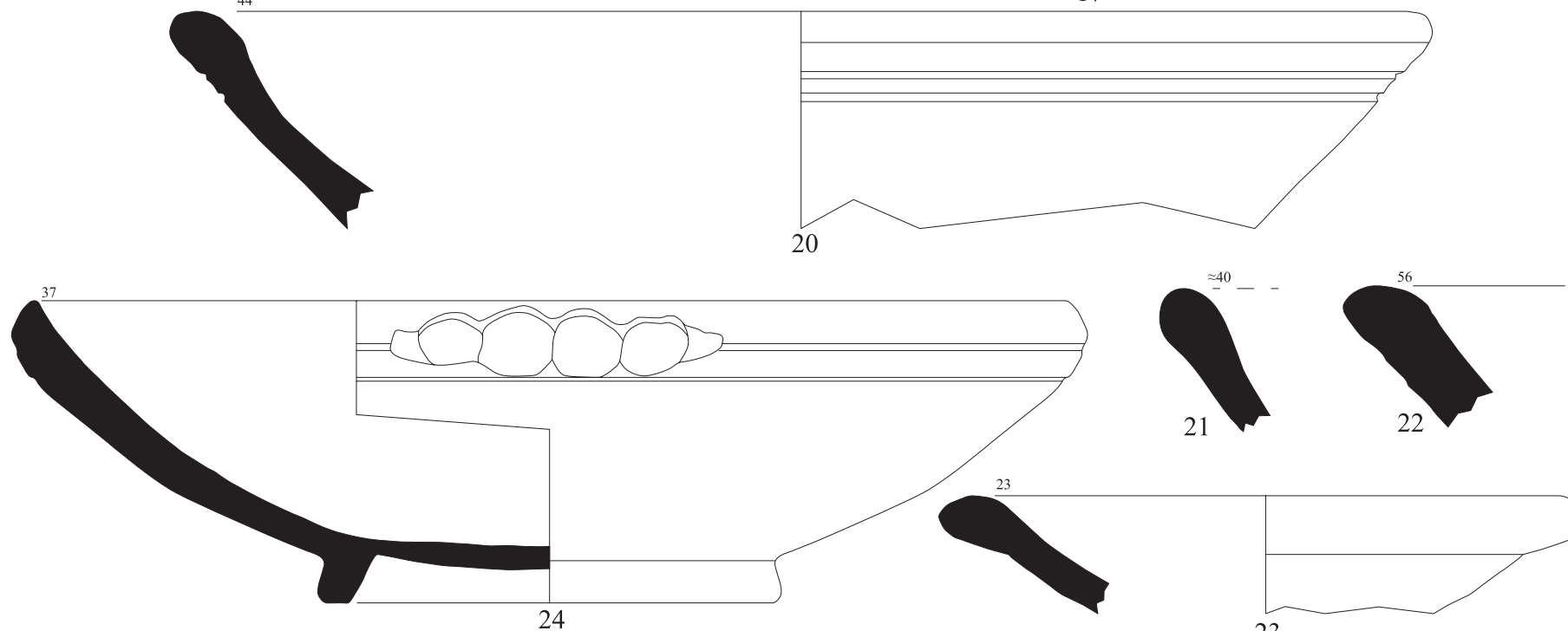

$1^{16,5}$
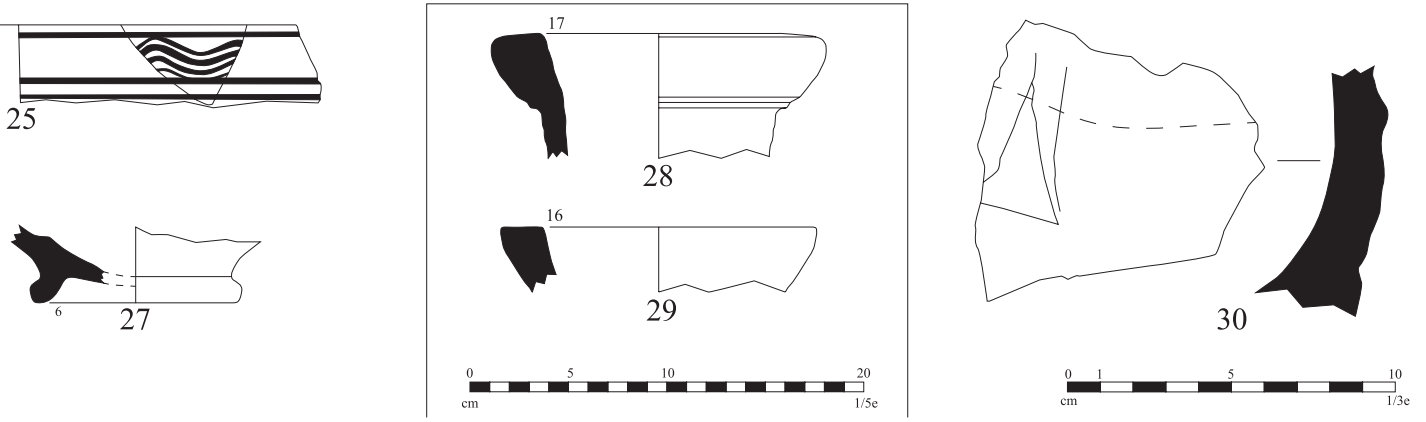

I 38 Mobilier céramique de la période $2 b(400-375$ av. J.-C.) - nos 1 à 17 céramiques à pâte claire massaliète ; $\mathrm{n}^{\circ} 18$ céramique pseudo-attique; $n^{\circ s} 19$ à 24 : mortier massaliète ; $n^{\circ s} 25$ à 27 : céramique grise monochrome ; nºs 28 à 30 : amphore massaliète

(A. Cauvin). 


\subsubsection{Céramique commune massaliète}

(fig. 38, nos 19 à 24)

Mieux représentés que dans les niveaux précédents, plusieurs types de mortiers se distinguent. Le mieux attesté est le type COM-MAS 621 avec une continuité entre sa panse et sa lèvre, celle-ci étant plus ou moins biseautée et bombée (fig. 38, nos 20 à 23 ).

Un mortier est entièrement conservé, de type COM-MAS 643: il présente des impressions digitées sur sa lèvre ainsi que deux profonds sillons dans la continuité de la panse, la lèvre présentant un fort biseau vers l'extérieur (fig. 38, n² 24).

\subsubsection{Amphore (fig. 38, $n^{o s} 28$ à 30)}

Seulement deux bords d'amphores massaliètes sont identifiés pour cette période. Il s'agit d'un bord de type bd5 en quart de cercle souligné par une mouluration (fig. 38, $\mathrm{n}^{\circ} 28$ ), et peut-être d'un bord bd6 dont la partie basse a été tronquée. Enfin, un graffite réalisé après cuisson, est inscrit sur le col d'une amphore: $\Delta \mathrm{I}$ (fig. $38, \mathrm{n}^{\circ} 30$ ). Cette pratique de marquer, par gravure ou à la peinture, les amphores est bien attestée, notamment à l'Île (Chausse-

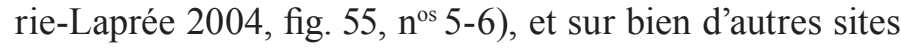
comme Saint-Pierre-les-Martigues (Chausserie-Laprée 2004, fig. 41, $\mathrm{n}^{\circ}$ 8), Lattes (Py et al. 2001, 237-239) ou même dans les Pyrénées-Orientales (Savarese 2011, fig. 6, $\left.\mathrm{n}^{\text {os }} 15-17\right)$.

\subsection{Période 2c (375/360 av. J.-C.)}

La période $2 \mathrm{c}$, qui correspond à la destruction brutale du site dans la première moitié du $\mathrm{IV}^{\mathrm{e}} \mathrm{s}$., très bien identifiée en stratigraphie, est également la mieux représentée dans les sondages méridionaux pour ce qui concerne le mobilier, avec 1653 fragments et 82 individus (fig. 39).

\subsubsection{Céramique non tournée provençale (fig. $40, n^{o s} 1$ à 16, fig. $41, n^{o s} 1$ à 6 )}

Les urnes, munies de lèvres plus ou moins déversées, sont toujours les formes les plus attestées (fig. $40, \mathrm{n}^{\text {os }} 1$ à 14 ). Il faut noter la présence de trois urnes de types CNT-PRO U4 qui présentent un décor différent avec un peignage qui débute au sommet de la panse (fig. $40, \mathrm{n}^{\text {os }} 1,2$ et 14 ). On peut également noter une urne de type CNT-PRO U5 avec une lèvre très déversée (fig. $40, \mathrm{n}^{\circ} 6$ ). Soulignons aussi l'urne complète de type CNT-PRO U4b présentant un décor original de points imprimés au milieu de sa panse (fig. $40, n^{\circ} 3$ ). Toujours plats, les fonds sont parfois peignés (fig. $40, \mathrm{n}^{\text {os }} 15$ et 16 ).
Au sein des formes ouvertes, parmi les coupes, il faut noter la présence d'une coupe carénée de type CNTPRO C $3 b$. Ses parois lissées et rentrantes présentent une pâte grise homogène aux inclusions nettement visibles (fig. 41, no 4).

Les jattes sont bien représentées et souvent très bien conservées (fig. $41, \mathrm{n}^{\text {os }} 1$ à 3 ). Une jatte de type CNT-PRO $\mathrm{J} 1$, présente un décor de fines incisions sur la lèvre ainsi qu'un petit verseur ménagé sur le bord. Des trous de réparations sont également constatés sur sa panse dans lesquels est encore insérée une agrafe en bronze (fig. 41, $\mathrm{n}^{\circ} 1$ ). Une deuxième jatte de même type présente un fond plat complètement blanchi et usé par le feu (fig. 41, nº 2 ). Ce type de jatte rappelle notamment par sa taille, sa forme et son traitement de surface (légèrement rugueux) un autre exemplaire découvert dans la partie nord du site (Damotte 2003, fig. 28, n⿳1 1). L'un des bords présente des parois épaissies renvoyant à des formes moins habituelles de type CNT-PRO J2b (fig. 41, nº 3 ). Enfin un bord arrondi et épaissi renvoie à des formes de mortiers en céramique non tournée (fig. $41, \mathrm{n}^{\circ} 6$ )

\subsubsection{Céramique non tournée à pâte claire \\ (fig. 41, $n^{\text {os }} 7$ à 10)}

La céramique non tournée à pâte claire semble ici être représentée par des coupes (fig. $41, \mathrm{n}^{\text {os }} 7$ à 9 ). Avec un diamètre inférieur à $13 \mathrm{~cm}$, elles sont assez petites, munies de parois bien polies et à flancs divergents. L'une se distingue par une fine rainure sur sa paroi externe juste sous la lèvre. Leur dégraissant est plus grossier que sur les productions massaliètes tournées.

\subsubsection{Céramique non tournée des rives de l'étang de Berre (fig. 41, $n^{\circ} 11$ )}

Une forme complète est représentée dans ces niveaux de destruction. Il s'agit d'une petite urne de type CNT-BER U1 au peignage soigné réalisé sur la totalité de la panse et s'arrêtant sur un col convergent. À l'instar d'autres urnes de cette production, la jonction col-panse est soulignée par un changement du sens du peignage ainsi que par une série d'incisions à la limite du col.

\subsubsection{Céramique à pâte claire massaliète \\ (fig. 42, $n^{o s} 1$ à 10)}

Les coupes sont les mieux représentées avec différents types (fig. 42, $\mathrm{n}^{\text {os }} 1$ à 4 ). En majorité peintes, elles sont du type CL-MAS 410 avec une lèvre aplatie et légèrement épaissie par rapport à la paroi (fig. $42, \mathrm{n}^{\text {os }} 1$ et 2 ). Deux autres bords, de type CL-MAS 428 et 425 , sont également peints (fig. $42, n^{\text {os }} 3$ et 4 ). 


\begin{tabular}{|c|c|c|c|c|c|c|c|c|c|c|c|c|c|}
\hline \multirow{2}{*}{ catégorie } & \multicolumn{3}{|c|}{ NFR } & \multicolumn{3}{|c|}{$\mathrm{NMI}$} & \multicolumn{3}{|c|}{ NBD } & \multirow{2}{*}{ forme } & \multirow{2}{*}{ type } & \multirow{2}{*}{$\begin{array}{l}\text { éléments } \\
\text { représentés }\end{array}$} & \multirow{2}{*}{ illustration } \\
\hline & $\mathrm{nb}$ & $\% /$ tot & $\% / g r p$ & $\mathrm{nb}$ & $\% /$ tot & $\% / g r p$ & $\mathrm{nb}$ & $\% /$ tot & $\% / g r p$ & & & & \\
\hline gris mono & 42 & 2,54 & 18,67 & 5 & 4,76 & 71,43 & 4 & 5 & 200 & \begin{tabular}{|l} 
coupe \\
couvercle \\
coupe carénée \\
jatte \\
oenochoé \\
\end{tabular} & $\begin{array}{l}\text { GR-MONO } 1 \\
\text { GR-MONO 16a } \\
\text { GR-MONO } 3 \\
\text { GR-MONO } 6 \\
\text { GR-MONO 8ac } \\
\end{array}$ & $\begin{array}{l}1 b \\
1 t \\
1 b, 1 f \\
1 b \\
1 c, 1 d \\
\end{array}$ & $\begin{array}{l}\text { fig. } 43, n^{\circ} 13 \\
\text { fig. } 43, n^{\circ} 14\end{array}$ \\
\hline cl.-mas & 167 & 10,1 & 74,22 & 17 & 16,19 & 242,86 & 12 & 15 & 600 & $\begin{array}{l}\text { coupe à une anse } \\
\text { coupe à anses } \\
\text { coupe à anses } \\
\text { olpé } \\
\text { cruche } \\
\text { cruche } \\
\text { autre } \\
\text { coupe à anses } \\
\text { cruche } \\
\text { coupe } \\
\end{array}$ & $\begin{array}{l}\text { CL-MAS } 410 \\
\text { CL-MAS } 425 \\
\text { CL-MAS } 428 \\
\text { CL-MAS } 522 \\
\text { CL-MAS } 525 \\
\text { CL-MAS } 542 \\
\text { CL-MAS ind. } \\
\text { CL-MAS ind. } \\
\text { CL-MAS ind. } \\
\text { CL-MAS ind. }\end{array}$ & $\begin{array}{l}2 b \\
1 b \\
2 b \\
1 c \\
1 b \\
2 b \\
1 f, 1 a \\
1 b, 1 a \\
2 f \\
2 b \\
\end{array}$ & $\begin{array}{l}\text { fig. } 42, n^{\circ 5} 1,2 \\
\text { fig. } 43, n^{\circ} 4 \\
\text { fig. } 42, n^{\circ} 3 \\
\text { fig. } 42, n^{\circ} 7 \\
\text { fig. } 42, n^{\circ} 5,6 \\
\text { fig. } 42, n^{\circ 5} 8,9\end{array}$ \\
\hline TOURNÉE FINE & 214 & 12,94 & 95,11 & 25 & 23,81 & 357,15 & 16 & 20 & 800 & & & & \\
\hline com-mas & 6 & 0,36 & 2,67 & 3 & 2,86 & 42,86 & 2 & 2,5 & 100 & $\begin{array}{l}\text { mortier } \\
\text { mortier } \\
\text { mortier }\end{array}$ & $\begin{array}{l}\text { COM-MAS } 632 \\
\text { COM-MAS 633a } \\
\text { COM-MAS ind. }\end{array}$ & $\begin{array}{l}1 b \\
1 b, 1 f \\
1 f\end{array}$ & $\begin{array}{l}\text { fig. } 42, n^{\circ} 16 \\
\text { fig. } 42, n^{\circ} 17 \\
\text { fig. } 42, n^{\circ} 15\end{array}$ \\
\hline TOURNÉE COM. & 6 & 0,36 & 2,67 & 3 & 2,86 & 42,86 & 2 & 2,5 & 100 & & & & \\
\hline CNT-Pro & 522 & 31,58 & 232 & 27 & 25,71 & 385,71 & 23 & 28,75 & 1150 & \begin{tabular}{|l} 
coupe \\
urne \\
mortier \\
autre \\
jatte \\
jatte \\
jatte \\
urne \\
urne \\
urne \\
\end{tabular} & $\begin{array}{l}\text { CNT-PRO C3b } \\
\text { CNT-PRO ind. } \\
\text { CNT-PRO ind. } \\
\text { CNT-PRO ind. } \\
\text { CNT-PRO J1 } \\
\text { CNT-PRO J1b } \\
\text { CNT-PRO J2b } \\
\text { CNT-PRO U4 } \\
\text { CNT-PRO U4b } \\
\text { CNT-PRO U5 }\end{array}$ & $\begin{array}{l}1 b \\
7 b, 5 f, 1 d \\
1 b \\
1 b, 1 f \\
1 c \\
1 c, 1 b \\
1 b \\
7 b, 1 f \\
1 c \\
1 b \\
\end{array}$ & 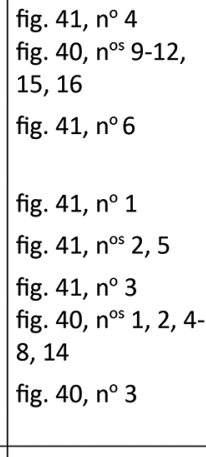 \\
\hline CNT-Gr & 1 & 0,06 & 0,44 & 1 & 0,95 & 14,29 & & & & & & & \\
\hline NON TOURNÉE & 580 & 35,09 & 257,77 & 34 & 32,37 & 485,71 & 29 & 36,25 & 1450 & & & & \\
\hline VAISSELLE & 800 & 48,39 & & 62 & 59,04 & & 47 & 58,75 & & & & & \\
\hline a-mas & 225 & 13,61 & 35,83 & 7 & 6,67 & 53,85 & 2 & 2,5 & 25 & $\begin{array}{l}\text { amphore } \\
\text { amphore } \\
\text { amphore }\end{array}$ & $\begin{array}{l}\text { A-MAS bd } 3 / 5 \\
\text { A-MAS bd5 } \\
\text { A-MAS ind. }\end{array}$ & $\begin{array}{l}1 b \\
1 b \\
2 f, 4 a\end{array}$ & $\begin{array}{l}\text { fig. } 43, n^{\circ} 10 \\
\text { fig. } 43, n^{\circ} 11 \\
\text { fig. } 43, n^{\circ} 9\end{array}$ \\
\hline AMPHORES & 225 & 13,61 & & 7 & 6,67 & & 2 & 2,5 & & & & & \\
\hline dolium & 628 & 37,99 & & 13 & 12,38 & & 8 & 10 & & $\begin{array}{l}\text { dolium à anse } \\
\text { dolium } \\
\text { dolium } \\
\text { dolium } \\
\text { dolium } \\
\text { dolium } \\
\text { dolium } \\
\end{array}$ & $\begin{array}{l}\text { DOLIUM bd3d } \\
\text { DOLIUM bd4a } \\
\text { DOLIUM bd5a } \\
\text { DOLIUM bd5c } \\
\text { DOLIUM bd7a } \\
\text { DOLIUM bd8c } \\
\text { DOLIUM ind. }\end{array}$ & $\begin{array}{l}1 b \\
1 b \\
1 b \\
1 b \\
1 b \\
2 b \\
1 b, 2 f\end{array}$ & $\begin{array}{l}\text { fig. } 43, n^{\circ} 9 \\
\text { fig. } 43, n^{\circ} 1 \\
\text { fig. } 43, n^{\circ} 2 \\
\text { fig. } 43, n^{\circ} 4 \\
\text { fig. } 43, n^{\circ} 3 \\
\text { fig. } 43, n^{\circ 5} 5,6 \\
\text { fig. } 43, n^{\circ 5} 7,8\end{array}$ \\
\hline TOTAL & 1653 & & & 82 & & & 57 & & & & & & \\
\hline
\end{tabular}

I 39 Période 2c (375-360 av. J.-C.) - Données quantitatives et typologiques du mobilier. 

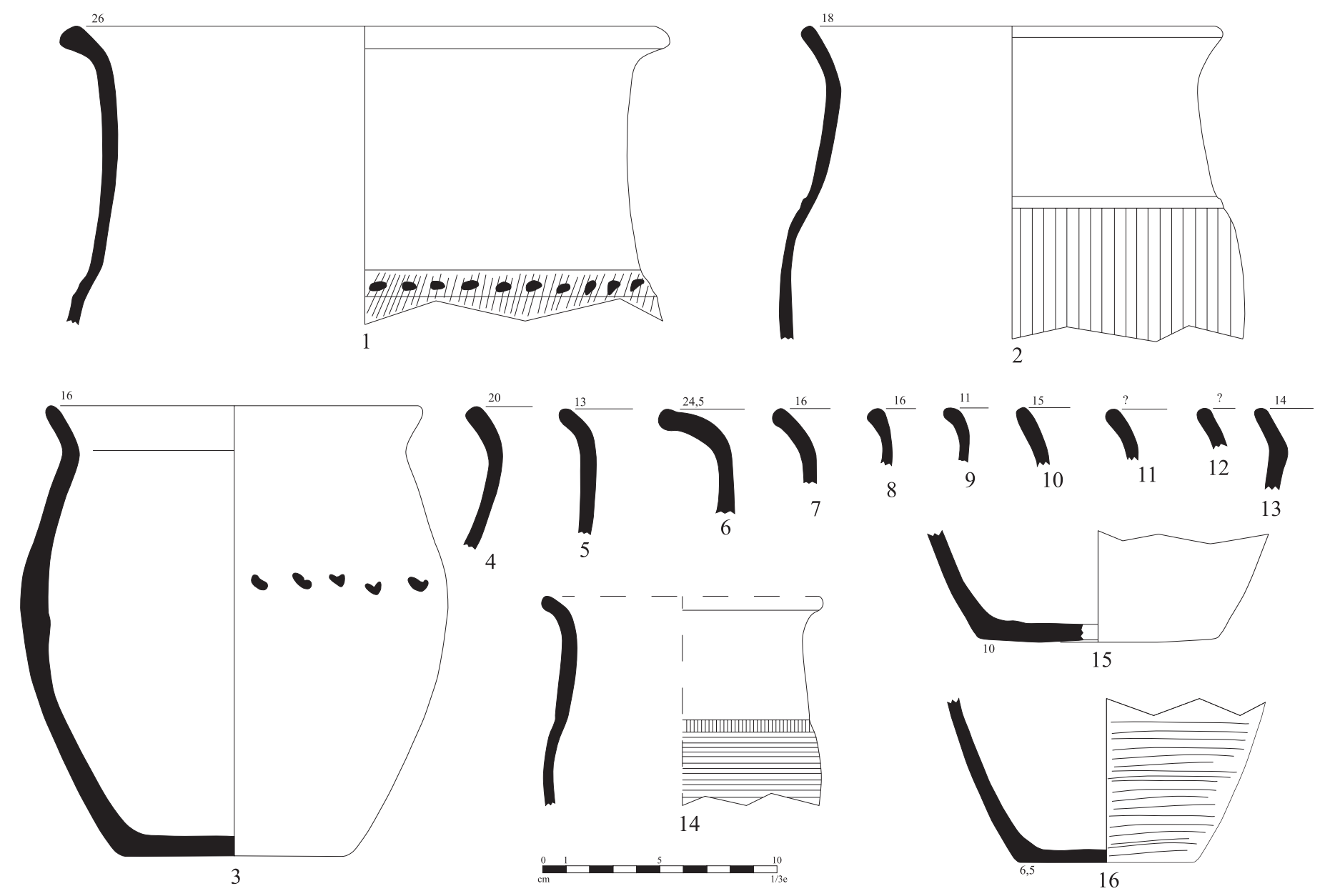

I 40 Mobilier céramique de la période 2c (375-360 av. J.-C.) - ns 1 à 16 : céramique non tournée provençale (A. Cauvin).

À côté de petites formes de cruches de type CL-MAS 542 (fig. $42, \mathrm{n}^{\text {os }} 5$ et 6 ), une petite olpé de type CL-MAS 522 apparaît complète (fig. $42, n^{\circ} 7$ ). Plusieurs fonds annulaires de cruches sont attestés (fig. $42, n^{\text {os }} 8$ et 9 ).

Un fragment de haut de panse est également à noter. Il est décoré par plusieurs lignes peintes de couleurs ocres verticales délimitées par une ligne ondée (fig. 42, $\mathrm{n}^{\circ} 10$ ). Il s'agit sans doute encore d'une production locale car aucune parallèle n'existe au sein des productions massaliètes (peut-être un exemplaire de cruche non tournée à pâte claire).

\subsubsection{Céramique pseudo-attique (fig. 42, $n^{\text {os }} 11$ et 12)}

Seuls deux pieds de coupes sont représentés au sein de cette catégorie. Le premier est un fragment de pied appartenant à une coupe, il offre une pâte orangée avec un vernis assez bien conservé et de vives arêtes. Le second, de type PSEUDO-AT 493, avait déjà été identifié (fig.37, $\mathrm{n}^{\circ}$ 12) (Chausserie-Laprée, Nin 1995, fig. 2, nº 9).

\subsubsection{Céramique commune massaliète (fig. 42, $n^{o s} 15$ à 17)}

Peu de formes sont clairement identifiées, si ce n'est un mortier de type COM-MAS 632 à marli plat pendant et un exemplaire de type COM-MAS 633a à marli bombé pendant, souligné par un sillon. Sa paroi extérieure est complètement usée et rongée.

\subsubsection{Dolium (fig. 43, $n^{o s} 1$ à 9)}

C'est durant cette période que les doliums sont les plus nombreux. Un seul a pu être restitué graphiquement (fig. $43, \mathrm{n}^{\circ} 1$ ). Il présente un bord de type bd4a avec une pâte rosée et un dégraissant moyen. Il montre des traces de peignages horizontaux sur la paroi intérieure. Le deuxième (fig. $43, \mathrm{n}^{\circ} 2$ ), de type bd5a, se distingue par son cordon sur le début de sa panse. Sa surface extérieure est complètement rongée, alors qu'à l'intérieur on peut voir des traces de peignage. Sa pâte est marron clair comprenant de nombreuses inclusions de chaux. Enfin quatre autres éléments de bords sont de type bd7a avec une lèvre et une panse très imposante (fig. $43, n^{\circ} 3$ ), de type bd5c 

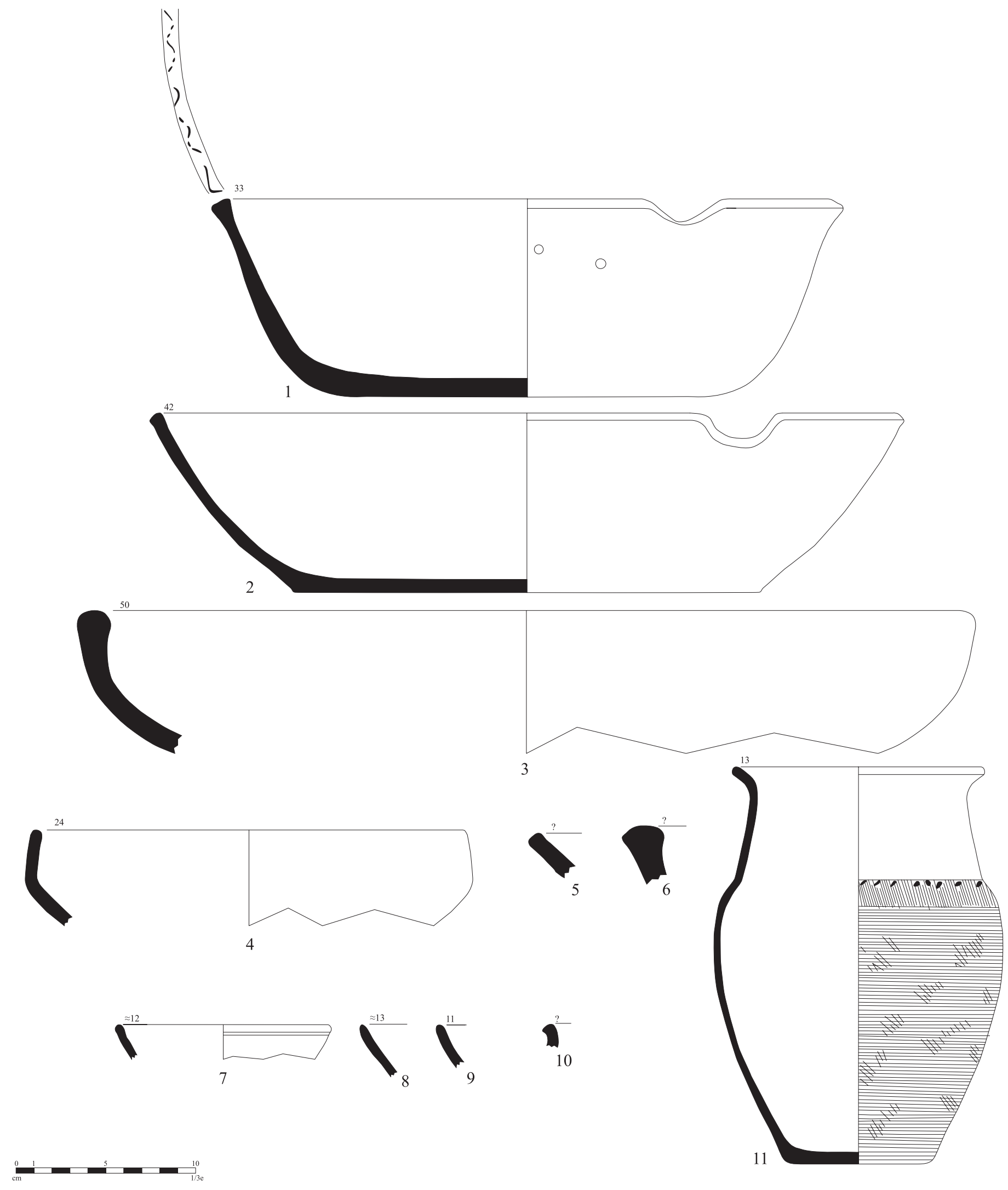

I 41 Mobilier céramique de la période 2c (375-360 av. J.-C.) - nºs 1 à 6 : céramique non tournée provençale ; nos 7 à 10 : céramique non tournée à pâte claire ;

$\mathrm{n}^{\circ} 11$ : céramique non tournée de l'étang de Berre

(A. Cauvin). 

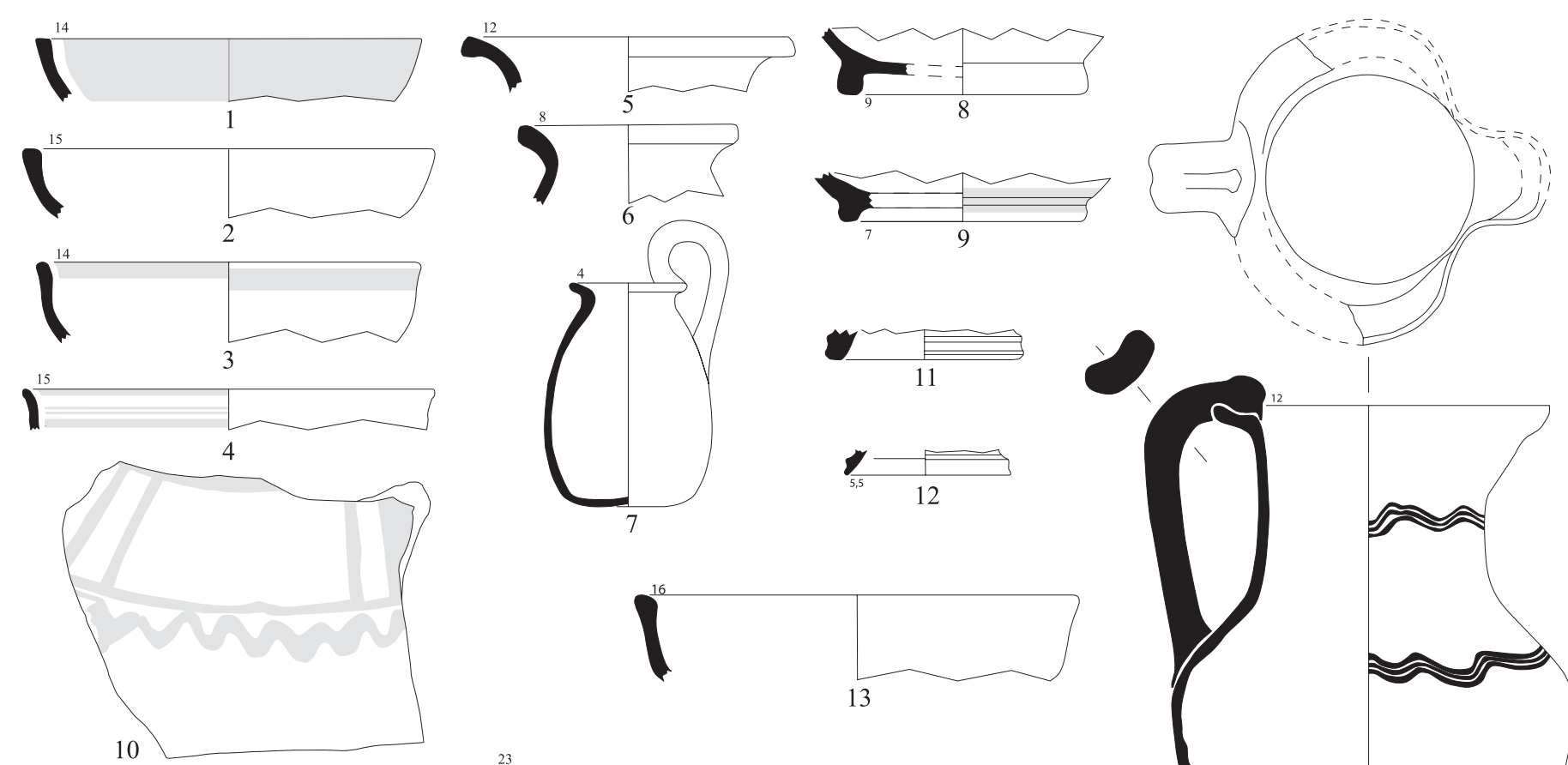

13
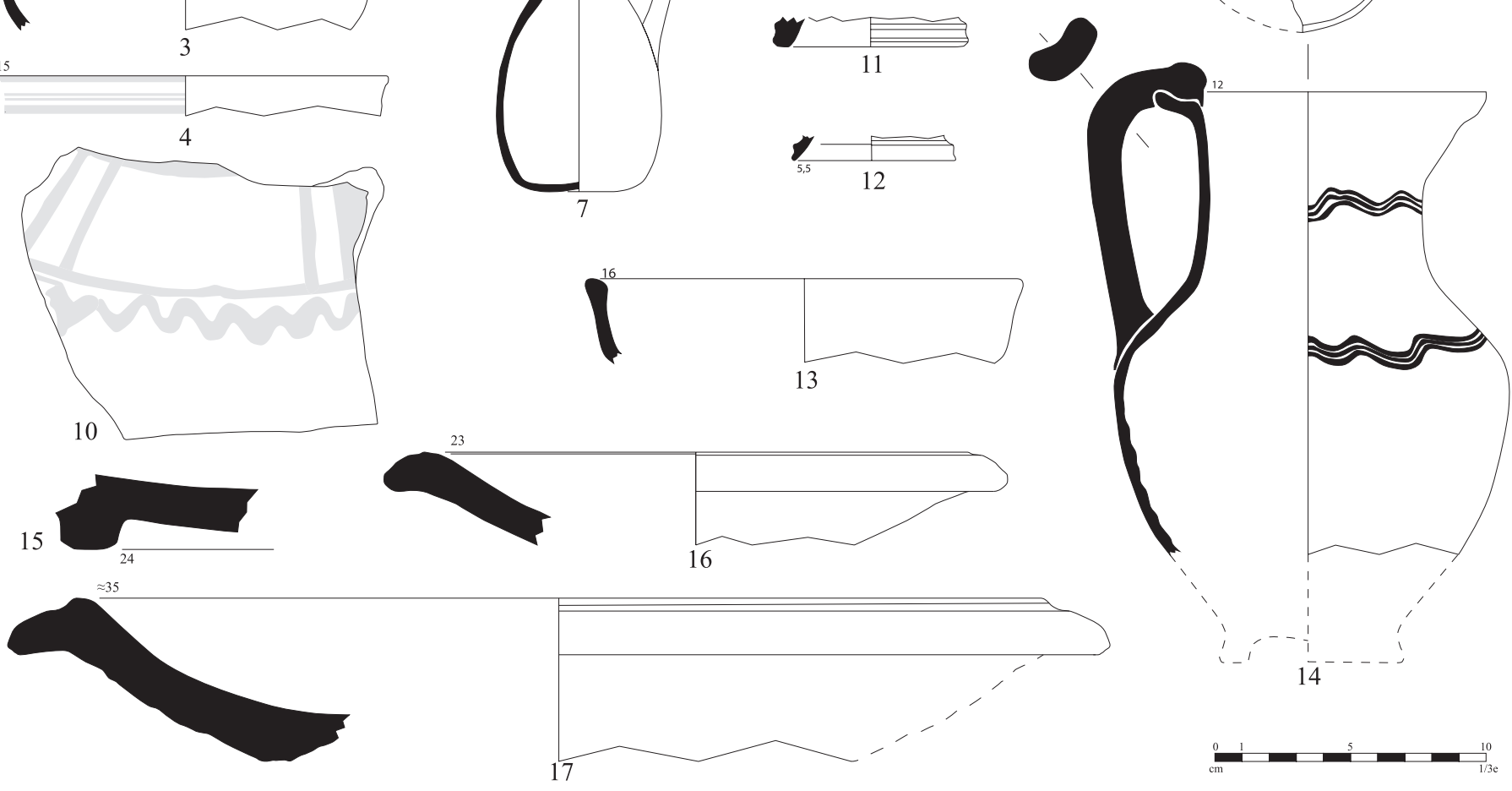

I 42 Mobilier céramique de la période $2 c(375-360$ av. J.-C. $)$ - $\mathrm{n}^{\circ \mathrm{s}} 1$ à 9 : céramique à pâte claire massaliète ; $n^{\circ} 10$ : céramique à pâte claire locale peinte; nos 11 et 12 : céramique pseudo-attique; nos 13 et $14:$ céramique grise monochrome ; $\mathrm{n}^{\text {os } 15}$ à $17:$ mortier massaliète (A. Cauvin).

comportant un méplat sur la lèvre épaissie par rapport à la paroi (fig. $43, \mathrm{n}^{\circ} 4$ ) et deux de type bd8c à la lèvre bien épaissie et au méplat incliné vers l'extérieur (fig. $43, \mathrm{n}^{\text {os }} 5$ et 6 ). Les fonds sont plats et l'un présente un cordon à la base du pied (fig. $43, n^{\text {os }} 7$ et 8 ).

Enfin, un dolium à anses est attesté dans la maison H3 (fig. 43, $\mathrm{n}^{\circ} 9$ ). Quelques exemplaires ont été signalés dans l'habitat de l'Île au sein d'autres îlots (Ratsimba 2005, pl. 21, 78; pl. 22, $79 ;$ pl. 23, 80). Si traditionnellement on a tendance à associer le dolium au stockage des céréales, certains auteurs relient les doliums à la viniculture. En effet, à Martigues comme à Coudounèu leur découverte a pu être associée à celle de rafles et de pépins de raisins. Cette attribution est ici renforcée par la forme spécifique de tels doliums, dont les anses, munies à la base d'un creux formé par l'impression du pouce dans la pâte, rappellent beaucoup celles des amphores massaliètes (Ratsimba 2005, 55; Chausserie-Laprée 2004, 179-183). Tous les exemplaires à anses sont localisés dans la région de l'étang de Berre (Ratsimba 2005, 50).

\subsubsection{Amphore (fig. 43, $n^{o s} 10$ à 12)}

Durant cette période, au moins trois amphores sont individualisées se caractérisant par un très bon état de conservation. De types différents, l'une assez trapue s'apparente au type A-MAS 2B caractérisée par de courtes anses (fig. 43, $\mathrm{n}^{\circ} 10$ ); la seconde, au col haut, est plutôt de type A-MAS 4 (fig. 43, $\mathrm{n}^{\mathrm{0}} 11$ ). La dernière de type A-MAS 3 présente un col convergent court et un bord en arc de cercle incliné vers l'extérieur (fig. $43, \mathrm{n}^{\circ} 12$ ). 

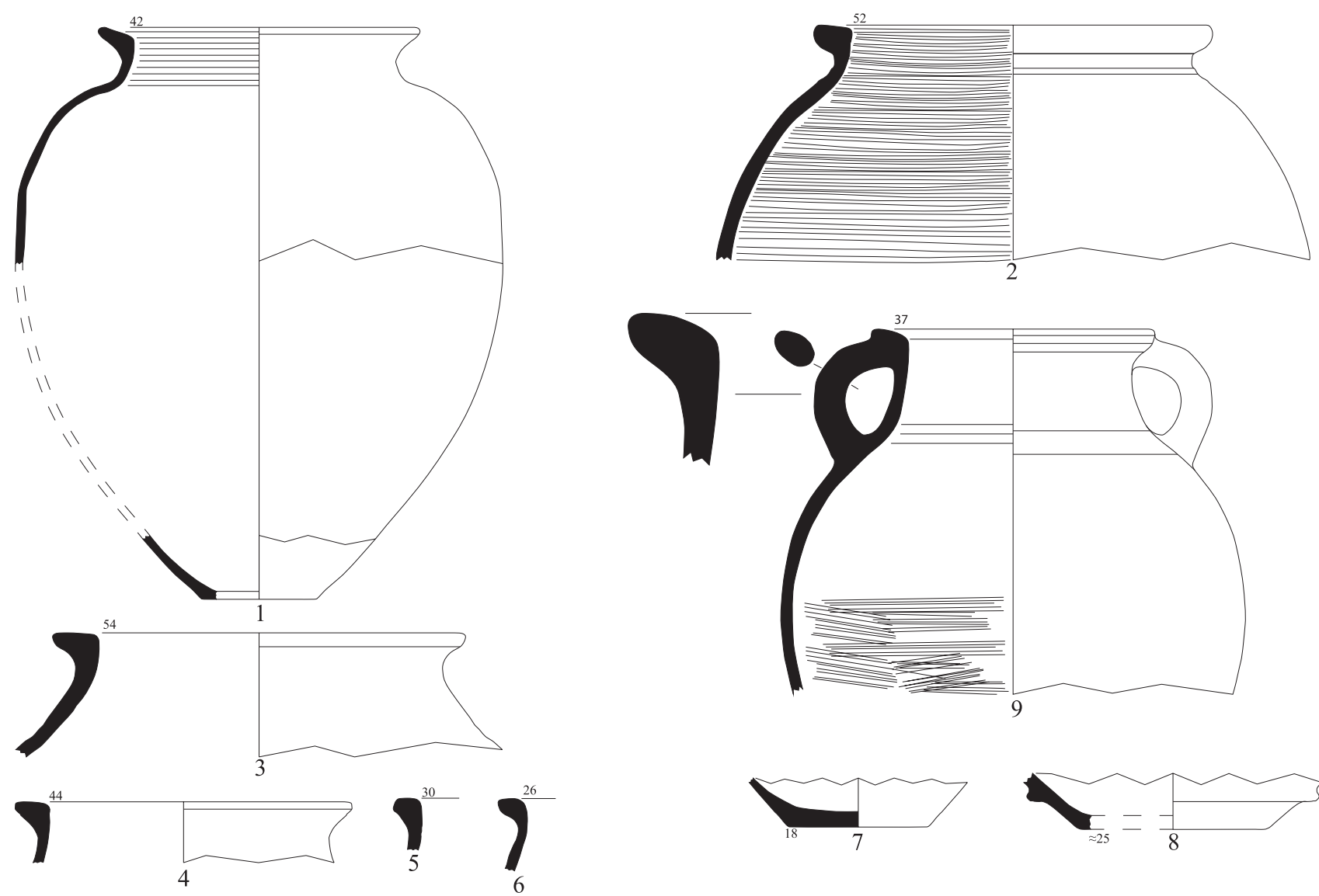

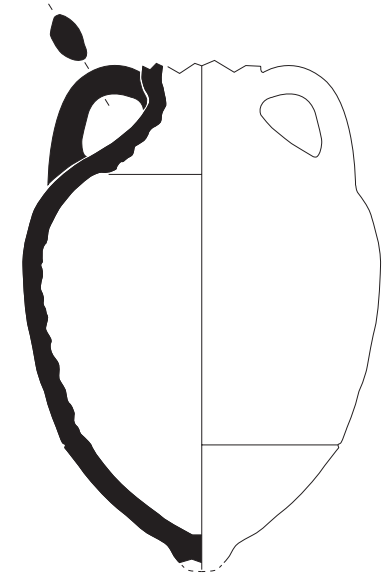

10

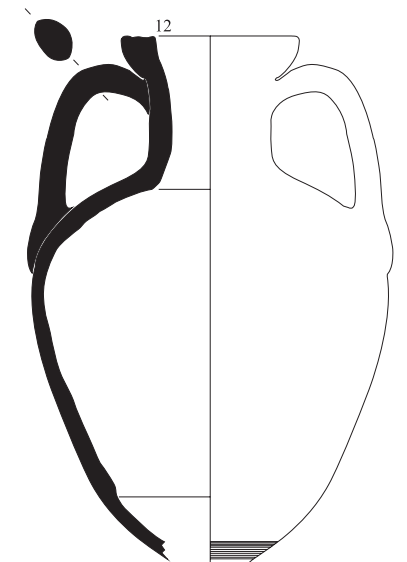

11
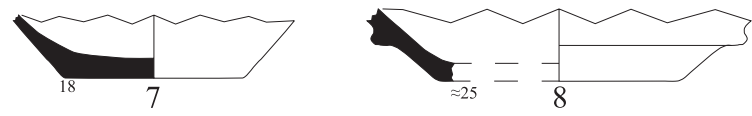

I 43 Mobilier céramique de la période 2c (375-360 av. J.-C.) - nos 1 à 9 : dolium (A. Cauvin) ; ns 10 à 12 : amphore massaliète (J.-Cl. Bardzakian - Service Archéologie de la Ville de Martigues).

\subsection{Période $3 a\left(360-325\right.$ av. J.-C.) (fig. 44, $n^{o s} 1$ à 6$)$}

La période 3a se caractérise par un retour à un répertoire céramique plus traditionnel interprété comme une forme de récession économique. Cette phase est très mal représentée dans la tranchée 8903 , très peu d'US y ayant été clairement attachées (fig. 45).
Cependant, quelques formes intéressantes sont à noter: un pied annulaire pour une probable cruche en céramique non tournée provençale (fig. $44, \mathrm{n}^{\circ} 2$ ); un bord de coupe en céramique non tournée à pâte grise, qui semble se distinguer des productions locales (fig. $44, \mathrm{n}^{\circ} 3$ ).

Il faut également souligner la présence de deux bords de dolium de deux types, bd5a et bd8d (fig. 44 , n ${ }^{\text {os }} 4$ et 5). Enfin, un fond très épais présentant des traces digitées se caracté- 
rise par sa pâte hétérogène dans laquelle sont visibles de très grosses particules de chamotte (fig. $44, n^{\circ} 6$ ).

\subsection{Période $3 b$ (325-200 av. J.-C.)}

La période $3 \mathrm{~b}$ se distingue par l'apparition des productions italiques. Elle est surtout présente dans les maisons H3, H4 et H5 (fig. 46).

\subsubsection{Céramique non tournée provençale}

(fig. 44, $n^{\text {os }} 7$ à 24)

Moins présentes que dans les niveaux précédents, les urnes sont toujours en majorité de type CNT-PRO U4 avec un fond plat, peignées et décorées ou non (fig. $44, n^{\text {os }} 7$ à 13 ). Une urne à bords convergents est également à noter (fig. $44, \mathrm{n}^{\circ} 14$ ). De type CNT-PRO U3a, elle se différencie par sa lèvre aplatie. Il est possible qu'il puisse s'agir d'un petit dolium à l'instar d'un autre bord (fig. $44, n^{\circ} 25$ ). Enfin comme pour la période précédente, on voit des fonds annulaires de cruche en céramique

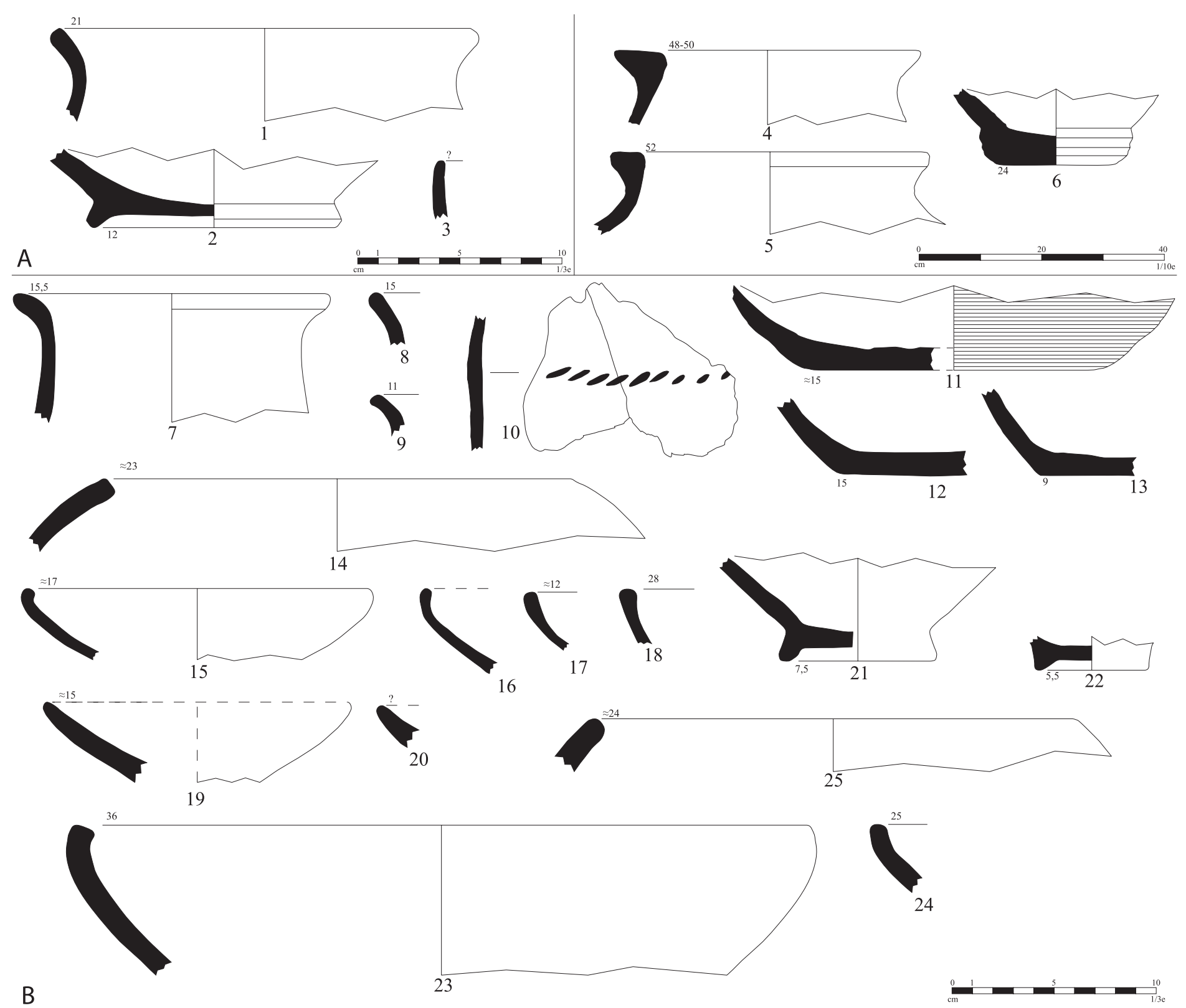

I 44 A - Mobilier céramique de la période $3 a\left(360-325\right.$ av. J.-C.) - $\mathrm{n}^{\circ \mathrm{s}} 1$ et 2 : céramique non tournée provençale ; $n^{\circ} 3$ : céramique à pâte claire massaliète; ns 4 à $6:$ dolium (A. Cauvin).

I B - Mobilier céramique de la période 3b (325-200 av. J.-C.) : $n^{\circ \mathrm{s}} 7$ à 24 : céramique non tournée provençale ; $n^{\circ} 25$ : dolium (A. Cauvin). 


\begin{tabular}{|c|c|c|c|c|c|c|c|c|c|c|c|c|c|}
\hline \multirow{2}{*}{ catégorie } & \multicolumn{3}{|c|}{ NFR } & \multicolumn{3}{|c|}{ NMI } & \multicolumn{3}{|c|}{ NBD } & \multirow{2}{*}{ forme } & \multirow[b]{2}{*}{ type } & \multirow{2}{*}{$\begin{array}{l}\text { éléments } \\
\text { représentés }\end{array}$} & \multirow{2}{*}{ illustration } \\
\hline & $\mathrm{nb}$ & $\% /$ tot & $\% / g r p$ & $\mathrm{nb}$ & $\% /$ tot & $\% / g r p$ & $\mathrm{nb}$ & $\% /$ tot & $\% / g r p$ & & & & \\
\hline gris mono & 1 & 0,82 & 1,69 & & & & & & & & & & \\
\hline cl.-mas & 1 & 0,82 & 1,69 & & & & & & & & & & \\
\hline pseudo-at & 1 & 0,82 & 1,69 & & & & & & & & & & \\
\hline TOURNÉE FINE & 3 & 2,46 & 5,07 & 0 & 0 & 0 & 0 & 0 & 0 & & & & \\
\hline com-mas & 3 & 2,46 & 5,08 & 1 & 11,11 & 20 & 1 & 20 & 33,33 & mortier & $\begin{array}{l}\text { COM-MAS 620- } \\
640 \\
\end{array}$ & $1 \mathrm{~b}$ & \\
\hline $\begin{array}{l}\text { TOURNÉE } \\
\text { COM. }\end{array}$ & 3 & 2,46 & 5,08 & 1 & 11,11 & 20 & 1 & 20 & 33,33 & & & & \\
\hline CNT-Pro & 52 & 42,62 & 88,14 & 3 & 33,33 & 60 & 1 & 20 & 33,33 & $\begin{array}{l}\text { autre } \\
\text { urne } \\
\text { urne }\end{array}$ & $\begin{array}{l}\text { CNT-PRO ind. } \\
\text { CNT-PRO ind. } \\
\text { CNT-PRO U4 }\end{array}$ & $\begin{array}{l}\mathrm{if} \\
1 \mathrm{f} \\
1 \mathrm{~b} \\
\end{array}$ & fig. $44, n^{\circ} 2$ \\
\hline CNT-Ind & 1 & 0,82 & 1,69 & 1 & 11,11 & 20 & 1 & 20 & 33,33 & coupe & CNT-PRO C3 & $1 \mathrm{~b}$ & fig. $44, n^{\circ} 3$ \\
\hline NON TOURNÉE & 53 & 43,44 & 89,83 & 4 & 44,44 & 80 & 2 & 40 & 66,66 & & & & \\
\hline VAISSELLE & 59 & 48,36 & & 5 & 55,55 & & 3 & 60 & & & & & \\
\hline a-mas & 27 & 22,13 & 100 & 2 & 22,22 & 100 & & & & amphore & A-MAS ind. & $1 a$ & \\
\hline AMPHORES & 27 & 22,13 & & 2 & 22,22 & & 0 & 0 & & & & & \\
\hline dolium & 36 & 29,51 & & 2 & 22,22 & & 2 & 40 & & $\begin{array}{l}\text { dolium } \\
\text { dolium }\end{array}$ & $\begin{array}{l}\text { DOLIUM bd5a } \\
\text { DOLIUM bd8d } \\
\end{array}$ & $\begin{array}{l}1 b, 1 f \\
1 b\end{array}$ & $\begin{array}{l}\text { fig. } 44, n^{\circ 5} 4,6 \\
\text { fig. } 44, n^{\circ} 5\end{array}$ \\
\hline TOTAL & 122 & & & 9 & & & 5 & & & & & & \\
\hline
\end{tabular}

I 45 Période 3a (360-325 av. J.-C.) - Données quantitatives et typologiques du mobilier.

\begin{tabular}{|c|c|c|c|c|c|c|c|c|c|c|c|c|c|}
\hline \multirow{2}{*}{ catégorie } & \multicolumn{3}{|c|}{ NFR } & \multicolumn{3}{|c|}{$\mathrm{NMI}$} & \multicolumn{3}{|c|}{ NBD } & \multirow{2}{*}{ forme } & \multirow{2}{*}{ type } & \multirow{2}{*}{\begin{tabular}{|c|} 
éléments \\
représentés \\
\end{tabular}} & \multirow{2}{*}{ illustration } \\
\hline & $\mathrm{nb}$ & $\% /$ tot & $\% / g r p$ & $\mathrm{nb}$ & $\% /$ tot & $\% / g r p$ & $\mathrm{nb}$ & $\% /$ tot & $\% / g r p$ & & & & \\
\hline cl.-mas & 78 & 12,46 & 15,15 & 8 & 17,78 & 19,05 & 5 & 15,63 & 17,24 & $\begin{array}{l}\text { coupe } \\
\text { olpé } \\
\text { cruche } \\
\text { olpé } \\
\text { cruche } \\
\text { autre } \\
\end{array}$ & $\begin{array}{l}\text { CL-MAS } 233 \\
\text { CL-MAS } 522 \\
\text { CL-MAS } 525 \\
\text { CL-MAS ind. } \\
\text { CL-MAS ind. } \\
\text { CL-MAS ind. }\end{array}$ & $\begin{array}{l}1 c \\
1 b, 1 a \\
2 b \\
2 f \\
1 a \\
1 b \\
\end{array}$ & $\begin{array}{l}\text { fig. } 47, n^{\circ} 6 \\
\text { fig. } 47, n^{\circ} 3 \\
\text { fig. } 47, n^{\circ s} 1,2 \\
\text { fig. } 47, n^{05} 4,5 \\
\text { fig. } 47, n^{\circ} 7\end{array}$ \\
\hline camp-a & 13 & 2,08 & 2,52 & 4 & 8,89 & 9,52 & 3 & 9,38 & 10,34 & bol & CAMP-A 27a & $3 b, 1 f$ & fig. $47, n^{\text {os }} 11-14$ \\
\hline TOURNÉE FINE & 102 & 16,3 & 19,81 & 15 & 33,34 & 35,71 & 11 & 34,39 & 37,92 & & & & \\
\hline com-mas & 2 & 0,32 & 0,39 & 1 & 2,22 & 2,38 & & & & mortier & COM-MAS ind. & 1f & fig. $47, n^{\circ} 15$ \\
\hline $\begin{array}{l}\text { TOURNÉE } \\
\text { COM. }\end{array}$ & 2 & 0,32 & 0,39 & 1 & 2,22 & 2,38 & 0 & 0 & 0 & & & & \\
\hline CNT-Pro & 411 & 65,65 & 79,81 & 26 & 57,78 & 61,9 & 18 & 56,25 & 62,07 & $\begin{array}{l}\text { coupe } \\
\text { coupe } \\
\text { coupe } \\
\text { annulaire } \\
\text { urne } \\
\text { jatte } \\
\text { coupe } \\
\text { jatte } \\
\text { urne } \\
\text { urne } \\
\end{array}$ & $\begin{array}{l}\text { CNT-PRO C1a ? } \\
\text { CNT-PRO C2a } \\
\text { CNT-PRO C3a } \\
\text { CNT-PRO ind. } \\
\text { CNT-PRO ind. } \\
\text { CNT-PRO ind. } \\
\text { CNT-PRO ind. } \\
\text { CNT-PRO J2a } \\
\text { CNT-PRO U3a } \\
\text { CNT-PRO U4 }\end{array}$ & $\begin{array}{l}2 b \\
2 b \\
2 b \\
2 f \\
5 b, 4 f \\
1 b \\
1 b \\
1 b \\
1 c \\
3 b, 1 f, 1 d \\
\end{array}$ & $\begin{array}{l}\text { fig. } 44, n^{\text {os }} 19,20 \\
\text { fig. } 44, n^{\circ 5} 17,18 \\
\text { fig. } 44, n^{\text {os }} 15,16 \\
\text { fig. } 44, n^{\text {os }} 21,22 \\
\text { fig. } 44, n^{\text {os }} 12,13 \\
\text { fig. } 44, n^{\circ} 24 \\
\text { fig. } 44, n^{\circ} 23 \\
\text { fig. } 44, n^{\circ} 14 \\
\text { fig. } 44, n^{\text {os }} 7-11\end{array}$ \\
\hline NON TOURNÉE & 411 & 65,65 & 79,81 & 26 & 57,78 & 61,9 & 18 & 56,25 & 62,07 & & & & \\
\hline VAISSELLE & 515 & 82,27 & & 42 & 93,34 & & 29 & 90,64 & & & & & \\
\hline a-mas & 48 & 7,67 & 100 & 1 & 2,22 & 100 & 1 & 3,13 & 100 & amphore & A-MAS ind. & $1 \mathrm{~b}$ & \\
\hline AMPHORES & 48 & 7,67 & & 1 & 2,22 & & 1 & 3,13 & & & & & \\
\hline dolium & 63 & 10,06 & & 2 & 4,44 & & 2 & 6,25 & & $\begin{array}{l}\text { dolium } \\
\text { dolium }\end{array}$ & \begin{tabular}{|l|} 
DOLIUM bd1b \\
DOLIUM ind.
\end{tabular} & $\begin{array}{l}1 \mathrm{~b} \\
1 \mathrm{~b} \\
\end{array}$ & \\
\hline
\end{tabular}

I 46 Période 3b (325-200 av. J.-C.) - Données quantitatives et typologiques du mobilier. 
non tournée (fig. $44, \mathrm{n}^{\text {os }} 21$ et 22). Le premier est percé d'un trou en son centre, montrant sans doute un remploi (comme couvercle?).

Les coupes sont plus nombreuses. Elles sont de type CNT-PRO C3a avec un flanc divergent et une lèvre légèrement rentrante (fig. $44, \mathrm{n}^{\text {os }} 15$ et 16), et de type CNT-PRO C2 à flancs divergents (fig. 44, $\mathrm{n}^{\text {os }} 17$ et 18). Deux autres bords de coupe, à flancs rectilignes et surface soigneusement polie, sont également à noter. L'orientation de ces vases est difficile à définir et il pourrait aussi s'agir de couvercles (fig. $44, \mathrm{n}^{\text {os }} 19$ et 20 ).

Enfin des jattes sont également attestées (fig. 44, $\mathrm{n}^{\text {os }} 23$ et 24). L'une d'entre elles, de type CNTPRO J2a, présente des parois et une lèvre irrégulière ainsi qu'une pâte grise au dégraissant assez grossier (fig. 44, $\mathrm{n}^{\circ} 23$ ).

\subsubsection{Céramique à pâte claire massaliète (fig. 47, $n^{o s} 1 \grave{a} 7$ )}

Les formes ouvertes de céramique à pâte claire massaliète semblent peu à peu disparaître durant cette période. Les éléments majoritaires sont des formes fermées, cruches ou olpés (fig. $47, \mathrm{n}^{\text {os }} 1$ à 5). Parmi les formes ouvertes, l'élément le mieux conservé est un plat de grand diamètre $(27 \mathrm{~cm})$, à la pâte grise homogène. Son pied, haut et anguleux, est percé peut-être pour la suspension du vase. Ses bords sont relevés et légèrement convergents et sa panse présente des traces de peinture rouge-orangé. Sa forme générale rappelle le type CL-MAS 233 ou 234 (fig. 47, nº 6).

\subsubsection{Céramique de l'atelier des petites estampilles (fig. 47, $n^{o s} 8$ à 10)}

Nouveauté de cette période, il faut noter l'arrivée des céramiques de l'atelier des petites estampilles qui se limitent ici à une seule forme, les bols de type 2783 à bord convergent. Le premier bol présente sur le fond une rosette centrale à seize pétales. Ce type de timbre semble assez rare et apparaît très peu attesté. En Italie, quelques attestations sont mentionnées mais le timbre est toujours en creux (Morel 1965, $\mathrm{n}^{\circ} 213$; Morel 1969, fig. 8-b). En Catalogne, ce type de timbre est attesté, mais cette fois-ci en relief, sur un fond de bol dans une composition à quatre timbres (Sanmarti-Grego 1973, fig. 9, n 1 ) ou dans une disposition centrale (Sanmarti-Grego

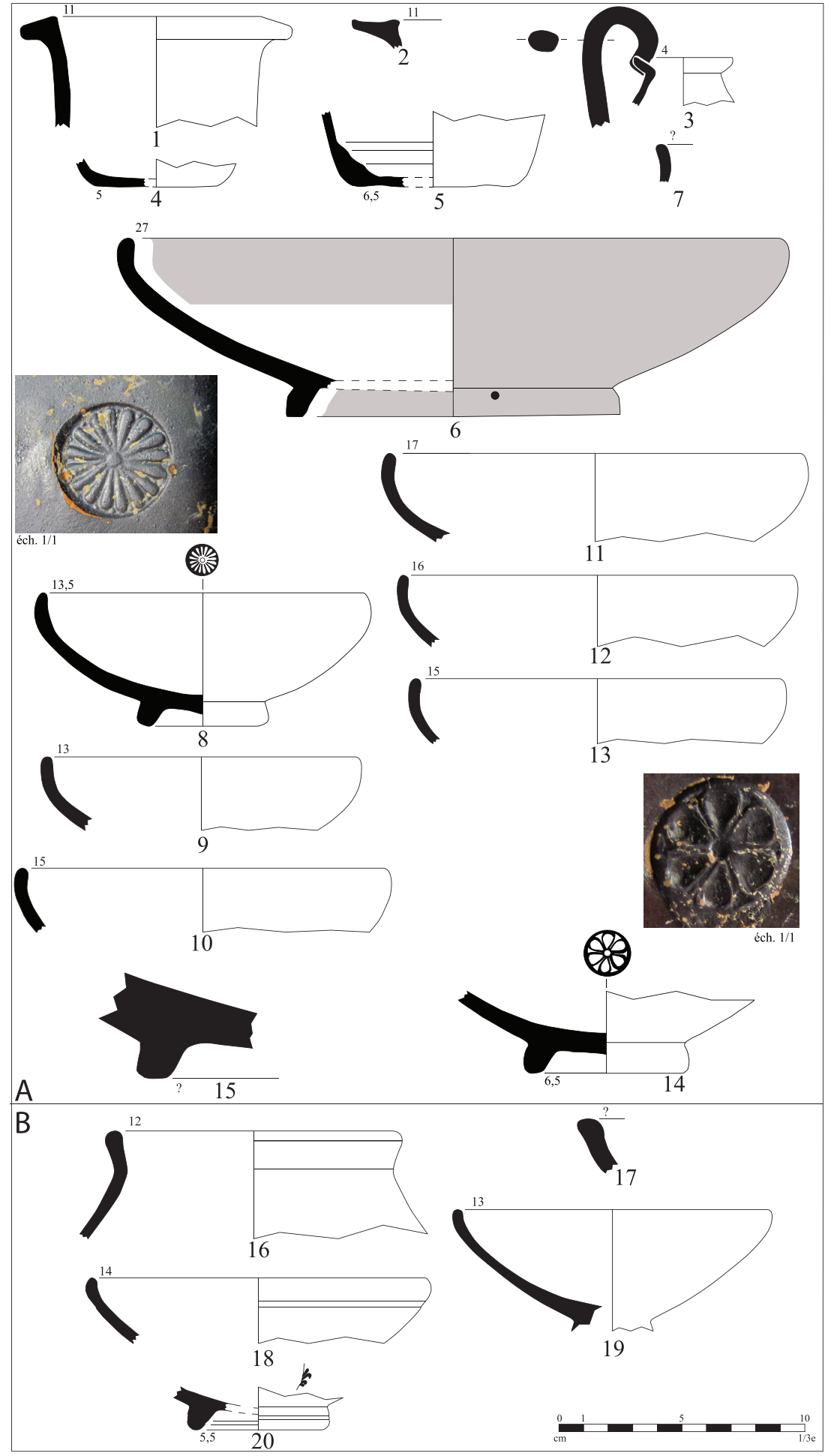

47 A Mobilier céramique de la période 3b (325-200 av. J.-C.) : nos 1 à 7 : céramique à pâte claire massaliète ;

$\mathrm{n}^{\text {os }} 8$ à 10 : céramique dite de l'atelier des petites estampilles; nos 11 à 14 : céramique campanienne $A$; $\mathrm{n}^{\circ} 15$ : fond de mortier massaliète (A. Cauvin).

I B Mobilier céramique de la période 4 (200-190 av. J.-C.) : $n^{\circ} 16$ et 17 : céramique non tournée provençale ; n०18: céramique campanienne $A$;

nos 19 et 20 : céramique de l'atelier des petites estampilles (A. Cauvin). 
1973, fig. $8, \mathrm{n}^{\circ}$ 2). Ses bords sont légèrement convergents et ses parois présentent de petites facettes. Sur la paroi externe du fond réservé, on distingue des traces de doigts qui sont la marque de l'immersion dans le bain d'argile pour appliquer l'engobe. Tous les bols ont un vernis très épais, aux reflets bleu vert.

\subsubsection{Céramique campanienne A (fig. 47, $n^{o s} 11$ à 14)}

Une seule forme est représentée parmi la céramique campanienne. Il s'agit des coupes de type CAMP-A 27b. La forme de ces coupes se distingue de celles de l'atelier des petites estampilles par une vasque plus profonde et un vernis d'une qualité légèrement inférieure (fig. $47, \mathrm{n}^{\text {os }} 11$ à 14). Un fond, qui se distingue par la couleur rosée de sa pâte, se caractérise par une estampille à six pétales séparés par des nervures aux extrémités bouletées (fig. $47, \mathrm{n}^{\circ} 14$ ). Le fond externe est réservé et on peut noter un bombement central, ce que J.-P. Morel appelait un «ombilic de tournassage» (Morel 1969, 62). Sur les flancs du pied, à l'instar des céramiques de l'atelier des petites estampilles, on peut noter le négatif des doigts du potier.

\subsection{Période 4 (200-190 av. J.-C.)}

Pour cette dernière période d'occupation du premier village de l'Île de Martigues, peu de vestiges en place ont été mis au jour dans le sondage 8903 . Il en va de même pour la céramique (fig. 48).

Cependant, parmi les quelques éléments de céramique non tournée provençale, on peut noter l'apparition d'une nouvelle forme d'urne sans col (fig. 47, $\mathrm{n}^{\circ} 16$ ).
Une coupe CAMP-A 27b est aussi attestée. Elle se différencie des productions précédentes par son léger sillon sous la lèvre (fig. 47, $\mathrm{n}^{\circ}$ 18). Un bol PET-EST 2783, présente de toutes petites facettes et une pâte rouge homogène (fig. 47, $n^{\circ} 19$ ). Enfin, on remarquera un fond avec un reste d'estampille à palmette partiellement conservée, présentant sur le pied de vives arêtes (fig. $47, \mathrm{n}^{\circ} 20$ ).

\subsection{Conclusion de l'approche typologique}

Au terme de cette étude, peut-on voir des différences et des évolutions typologiques au sein des catégories de mobilier céramique mises en évidence? Évidemment, pour répondre à cette question, nous nous heurtons à l'écueil de la représentativité parfois insuffisante de nos échantillons. C'est en particulier le cas pour la période $3 \mathrm{a}$.

Toutes périodes confondues, la céramique non tournée provençale apparaît largement majoritaire. Les urnes, forme principale de ce répertoire, sont attestées au cours de chacune des périodes d'occupation. Durant les périodes 1 et $2 \mathrm{a}$, les urnes présentent des profils aux bords peu marqués et à la panse peu galbée. Le contact col/panse est souvent souligné par un décor incisé ou imprimé. À la période $2 b$, la lèvre semble être plus marquée, plus déversée, le galbe de la panse est également plus visible souligné parfois par un ressaut (fig. 37 $\mathrm{n}^{\circ} 12$ ). C'est d'ailleurs à cette période qu'apparaissent les imitations des productions de l'étang de Berre, qui reprennent leur forme caractéristique, mais qui s'en distinguent par des décors moins fins et une cuisson moins élevée et maîtrisée (Damotte 2003, 210). Concernant les autres formes, il est difficile d'aller au-delà de généralités, vu la faiblesse du nombre

\begin{tabular}{|c|c|c|c|c|c|c|c|c|c|c|c|c|c|}
\hline \multirow{2}{*}{ catégorie } & \multicolumn{3}{|c|}{ NFR } & \multicolumn{3}{|c|}{ NMI } & \multicolumn{3}{|c|}{ NBD } & \multirow{2}{*}{ forme } & \multirow{2}{*}{ type } & \multirow{2}{*}{$\begin{array}{l}\text { éléments } \\
\text { représentés }\end{array}$} & \multirow{2}{*}{ illustration } \\
\hline & $\mathrm{nb}$ & \%/tot & $\% / g r p$ & $\mathrm{nb}$ & $\% /$ tot & $\% / g r p$ & $\mathrm{nb}$ & $\% /$ tot & $\% / g r p$ & & & & \\
\hline cl.-mas & 9 & 8,49 & 9,28 & 1 & 11,11 & 11,11 & & & & autre & CL-MAS ind. & $1 \mathrm{f}$ & \\
\hline petest & 6 & 5,66 & 6,19 & 1 & 11,11 & 11,11 & 1 & 20 & 20 & bol & PET-EST 2783 & $1 b, 1 f$ & fig. $47, n^{\circ s} 19,20$ \\
\hline camp-a & 6 & 5,66 & 6,19 & 2 & 22,22 & 22,22 & 1 & 20 & 20 & $\begin{array}{l}\text { bol } \\
\text { coupe }\end{array}$ & $\begin{array}{l}\text { CAMP-A 27a } \\
\text { CAMP-A ind. }\end{array}$ & $\begin{array}{l}1 b \\
1 f\end{array}$ & fig. $47, n^{\circ} 18$ \\
\hline TOURNÉE FINE & 21 & 19,81 & 21,66 & 4 & 44,44 & 44,44 & 2 & 40 & 40 & & & & \\
\hline NON TOURNÉE & 76 & 71,7 & 78,35 & 5 & 55,56 & 55,56 & 3 & 60 & 60 & & & & \\
\hline VAISSELLE & 97 & 91,51 & & 9 & 100 & & 5 & 100 & & & & & \\
\hline dolium & 9 & 8,49 & & & 1 & & & & & & & & \\
\hline
\end{tabular}

I 48 Période 4 (200-190 av. J.-C.) - Données quantitatives et typologiques du mobilier. 
d'individus. On note tout de même plusieurs tendances: la raréfaction des décors sur les jattes à partir de la fin du $\mathrm{IV}^{\mathrm{e}}$ siècle; le développement au III siècle de bords relevés, voire convergents pour les coupes, peut-être sous l'influence des apports méditerranéens (Marty 2005, 44-45).

En ce qui concerne la céramique non tournée des rives de l'étang de Berre, peut-être un peu sous-estimée dans nos décomptes, l'urne est logiquement très majoritaire. La quasidisparition de cette production après la destruction par incendie de la période $2 \mathrm{c}$ semble conforter l'hypothèse de la fin de sa production dans la deuxième moitié du IV $\mathrm{e}$ s. av. J.-C.

Quoique très minoritaires au sein du répertoire, les productions non tournées à pâte claire, urnes et aussi coupes, sont typiques de cette région et dénotent sans doute l'influence des productions tournées de Marseille.

La céramique à pâte claire massaliète mise au jour à Martigues ne présente pas de caractère particulier avec les formes les plus courantes du répertoire, principalement des coupes, des cruches et des olpés. S'agissant de formes très «classiques», elles ne montrent pas une évolution typologique notable.

Cependant quelques tessons et parmi eux deux fragments de la couche 1060 présentent une forme, mais surtout une pâte et un revêtement inhabituels dans les productions massaliètes. Certains chercheurs (rens. J.-C. Sourrisseau, M. Bats) ont émis l'hypothèse qu'ils pourraient appartenir, avec d'autres céramiques attestées à Saint-Blaise et sur l'oppidum de SaintPierre-les-Martigues, à une production de céramique claire fabriquée dans les environs de Martigues.

On rejoint ici une problématique propre à cette grande catégorie de céramique à pâte claire, qui comprend, outre les vases fabriqués à Marseille même, de nombreuses autres productions locales, identifiées aux marges de l'aire massaliète. Les céramiques à pâte claire du Languedoc occidental, dites ibéro-languedociennes) sont attestées dès le $\mathrm{V}^{\mathrm{e}}$ siècle av. J.-C. (Chazelles, Ugolini 2015). De même, au nord de la Provence, il faut citer les productions rhodaniennes, notamment repérées sur l'oppidum du Pègue dans la Drôme (Moulin 2014).

\section{Analyse des données}

\subsection{Typologie et chronologie}

Chacune des périodes d'occupation du site déterminées par les fouilles précédentes a été à nouveau identifiée dans le sondage 8903. En dépit de la relative faiblesse numérique de la collection étudiée, qui compte tout de même plus de 4000 fragments et 320 individus, ce qui frappe ici ce sont l'homogénéité et la cohérence du corpus étudié. Pour chaque période, les objets mis au jour et en particulier ceux dont la typologie est la mieux assurée (amphores de Marseille, céramique grise monochrome, céramique non tournée des rives de l'étang de Berre, céramiques à vernis noir) se sont avérés tout à fait en accord avec les datations déjà proposées. Cependant cette fouille, qui n'a livré aucun objet fournissant une datation absolue précise, pour les périodes anciennes en particulier ${ }^{18}$, n'a pas apporté d'élément nouveau pouvant affiner une chronologie parfois assez lâche. C'est particulièrement vrai pour les épisodes destructifs qui ont frappé le site à plusieurs reprises.

Ainsi la question de la date de l'épisode militaire, qui marque la période $2 \mathrm{c}$ et dont les traces sont bien visibles au sein des différents espaces mis au jour dans le sondage 8903, reste ouverte. Nous ne pouvons en l'état trancher entre une datation «basse» (vers 375/360 av. J.-C.) que nous privilégions ici sur la base des données céramologiques (Chausserie-Laprée, Nin 1995; Campenon, Chausserie-Laprée 2000; Damotte 2003; Chausserie-Laprée 2004) et une datation haute (vers 400/380 av. J.-C.,) proposée par B. Girard-Millereau (2010, 590) à partir du matériel métallique.

\subsection{Analyse quantitative}

À l'instar d'autres secteurs étudiés du site, la fouille de cette zone sud n'a livré qu'un petit nombre de catégories différentes de céramiques. La vaisselle est composée principalement de céramique non tournée $(69,06 \%$ des fragments) $)^{19}$. Ensuite, avec $26,01 \%$ du total, viennent les céramiques tournées marseillaises (céramique à pâte claire massaliète, mortiers massaliètes, céramique pseudo-attique). La céramique grise monochrome ne représente que $3,59 \%$ de la vaisselle, à peine plus que les vases d'importation méditerranéenne $(1,3 \%)$, représentés surtout par les importations à vernis noir d'Italie à partir de la fin du III' ${ }^{e}$ siècle (fig. 29). 
Pour ce qui est des amphores, on retrouve ici la domination écrasante des conteneurs marseillais qui représentent $15,66 \%$ des fragments de la céramique et 7,29\% des individus. Quelques éléments étrusques et italiques n'apparaissent que de manière anecdotique dans des couches au contexte par ailleurs incertain ou remanié, et que nous n'avons donc pas pu prendre en compte.

Ainsi, malgré des évolutions propres à telle ou telle période, c'est bien la dominante locale qui caractérise le mobilier de cette partie du site. Il confirme aussi que les échanges se font de manière quasi-exclusive avec le partenaire marseillais.

Quant au rapport entre vaisselle et mobilier amphorique ou de réserve, il reste à peu près équivalent quelles que soient les périodes étudiées, la vaisselle étant toujours nettement majoritaire (autour de $70 \%$ du mobilier). Seule la période $2 \mathrm{c}$ montre une dynamique différente avec une forte représentation $(30 \%)$ des doliums dans les niveaux d'incendie des maisons ${ }^{20}$ (fig. 49).

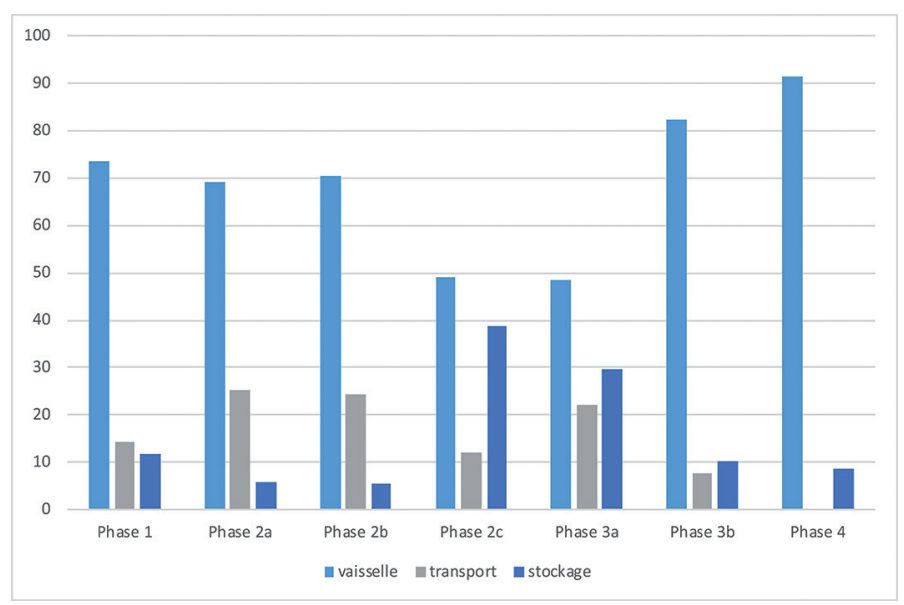

I 49 Proportions des groupes céramiques (\% sur total de fragments).

La question est de savoir si cette augmentation des vases de stockage durant la phase $2 \mathrm{c}$ repose sur la bonne conservation des doliums au sein du contexte de destruction ou si elle correspond à une augmentation réelle des capacités de stockage de l'habitat à ce moment précis de l'occupation du village. L. Damotte $(2003,213)$ constatait le même phénomène dans les îlots du nord du site avec des taux sensiblement identiques. Elle se demandait s'il y avait eu une volonté de regrouper les moyens de stockage en lien avec un moment de crise ou si cette augmentation du nombre de doliums était la conséquence du développement de ce type de récipients, à cause d'une production agricole accrue.
On doit s'intéresser aussi aux proportions de céramiques tournées et non tournées selon les périodes. Durant la période 1, la CNT est largement majoritaire en NFR (près de $80 \%$ ) et en NMI (environ 60\%). Puis ce rapport tend à s'équilibrer puisque, durant la période $2 \mathrm{~b}$, la CNT ne représente plus que $55 \%$ des fragments. Durant les périodes $3 \mathrm{~b}$ et 4 , cet équilibre est à nouveau rompu et la CNT domine de nouveau très largement la vaisselle avec près de $80 \%$ des fragments et environ $60 \%$ des individus (fig. 50, A-B).

On peut s'étonner des taux assez importants de céramique tournée surtout pour les périodes anciennes. Ces résultats diffèrent avec ceux mis en évidence dans la partie nord-ouest du village où la céramique non tournée apparaît bien plus abondante au sein de la vaisselle (Damotte 2003, fig. 35-36). Comment expliquer ces différences? Est-ce un problème de représentativité ou la partie sud du village marque-t-elle une dynamique différente?
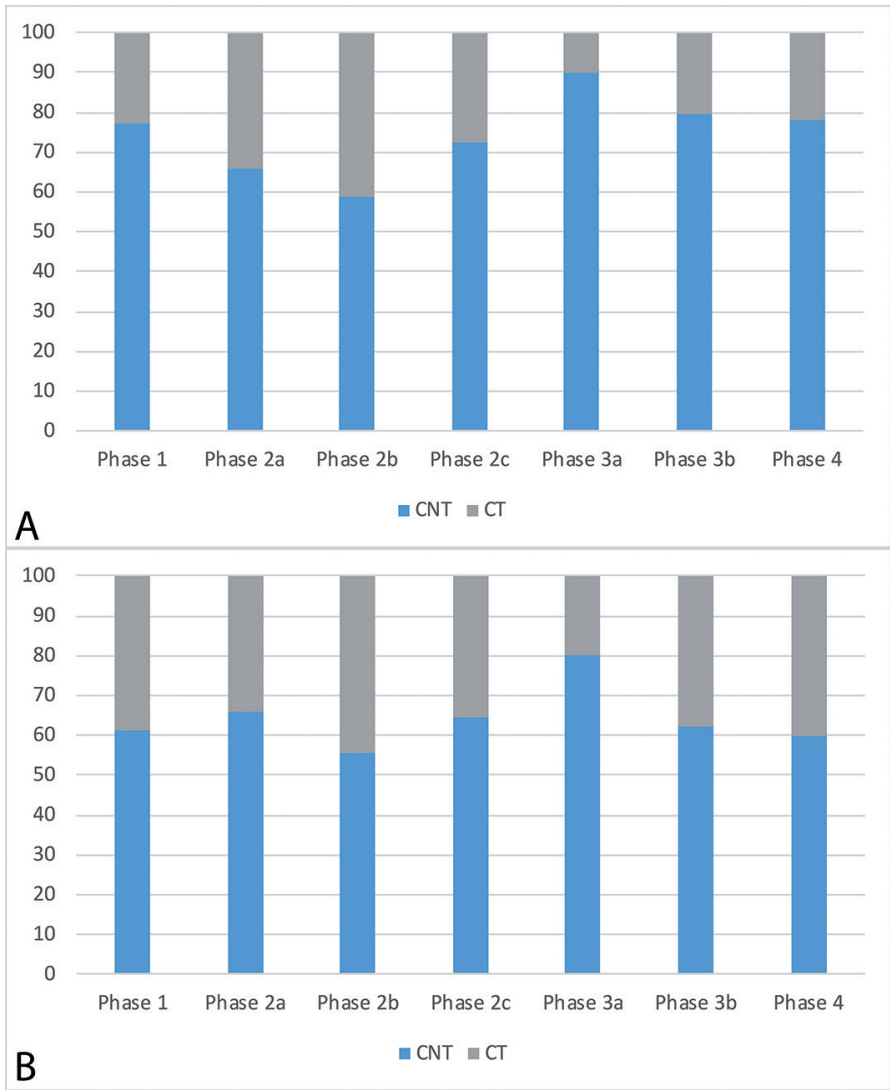

1 50 Proportions des céramiques non tournées et tournées.

- A - \% sur total des fragments de vaisselle.

I B - \% sur total des individus de vaisselle. 


\subsection{Quelques considérations sur la vaisselle du sondage 8903}

\subsubsection{Formes et répartition de la céramique non tournée}

En ce qui concerne la céramique non tournée, toutes périodes confondues, les productions domestiques, strictement locales, dites CNT-PRO, atteignent environ $90 \%$ des fragments (fig. 51, A et B). Comme lors des études précédentes, dans ce lot global des CNT, on constate une augmentation de la part des céramiques non tournée des rives de l'étang de Berre (CNT-BER) entre la période 1 et la période $2 b$, puisqu'elle représente alors plus de $10 \%$ des fragments de céramique non tournée ${ }^{21}$. Quant à la part des productions non tournées à pâte claire (CNT-CL), bien plus faible, elle tourne autour des 2 à $4 \%$ jusqu'au milieu du IV siècle pour disparaître complètement à partir de la période $3 \mathrm{a}$.

Les formes de céramique non tournée représentées ici, dans des proportions assez similaires à celles observées dans la partie nord-ouest du village, sont pratiquement limitées à quatre, avec dans l'ordre de leur importance: l'urne, dont les fonctions diverses expliquent la nette prépondérance; la

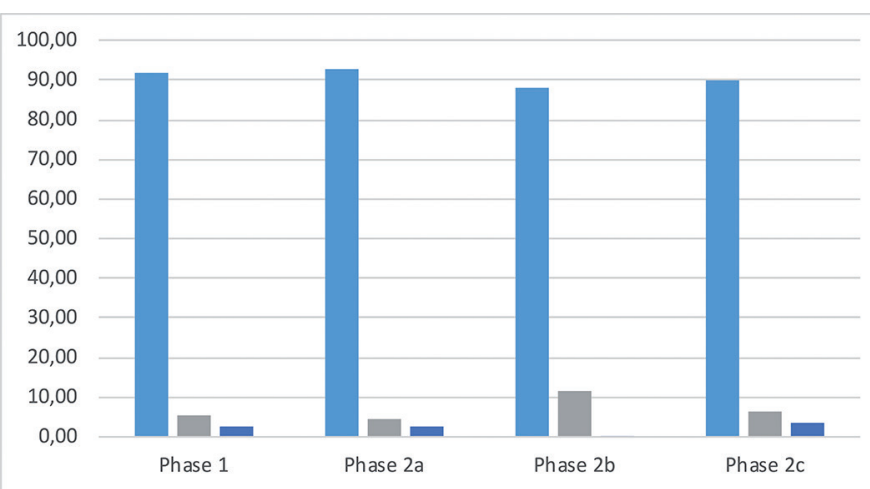

A

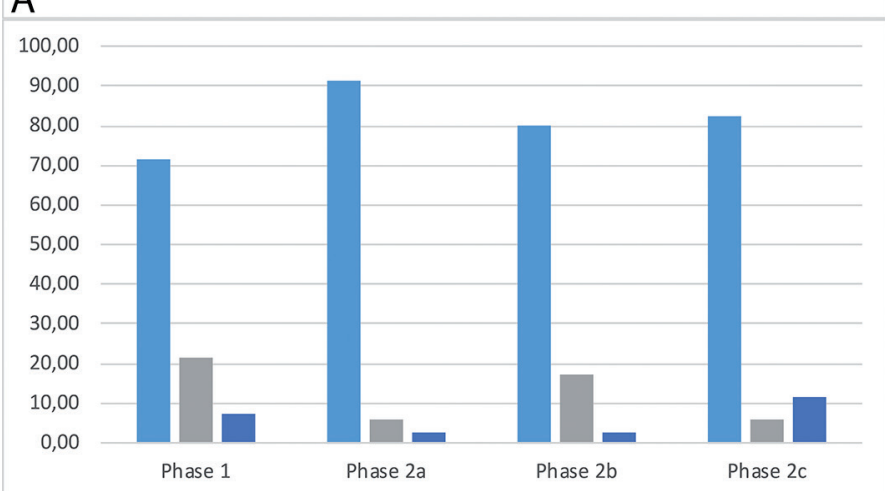

B

$\square$ CNT-PRO $\square$ CNT-BER $\square$ CNT-CL

I 51 Proportions des catégories de céramiques non tournées.

- A - \% sur total des fragments de céramiques non tournées.

I B - \% sur total des individus de céramiques non tournées. coupe ou le bol; la jatte; et enfin le mortier, copie avérée des productions communes massaliètes.

\subsubsection{Formes et répartition de la céramique tournée}

La céramique marseillaise (pâte claire, commune et pseudoattique) représente une écrasante majorité de la céramique tournée avec plus de $85 \%$ des fragments toutes périodes confondues et $70 \%$ des individus. La céramique grise monochrome est loin derrière avec seulement $8 \%$ des fragments et $20 \%$ des individus de la céramique tournée. Toutes les autres productions sont anecdotiques. Seules les productions italiques durant les deux dernières phases d'occupation du village apparaissent plus clairement, mais le corpus n'est pas suffisamment important pour bien apprécier cette apparition.

Sur la longue durée, il est intéressant de noter le fort déclin de la céramique fine massaliète lors de la période $3 \mathrm{~b}$ au profit de la céramique à vernis noir italique. En effet, la claire massaliète, qui lors de la période $2 \mathrm{c}$ représente plus de $60 \%$ des céramiques tournées en nombre d'individus, chute à $25 \%$ des individus de la céramique tournée lors de la phase suivante. Cette diminution apparait liée à l'arrivée des productions italiques qui, en nombre d'individus, deviennent même majoritaires (fig. 52, A et B).

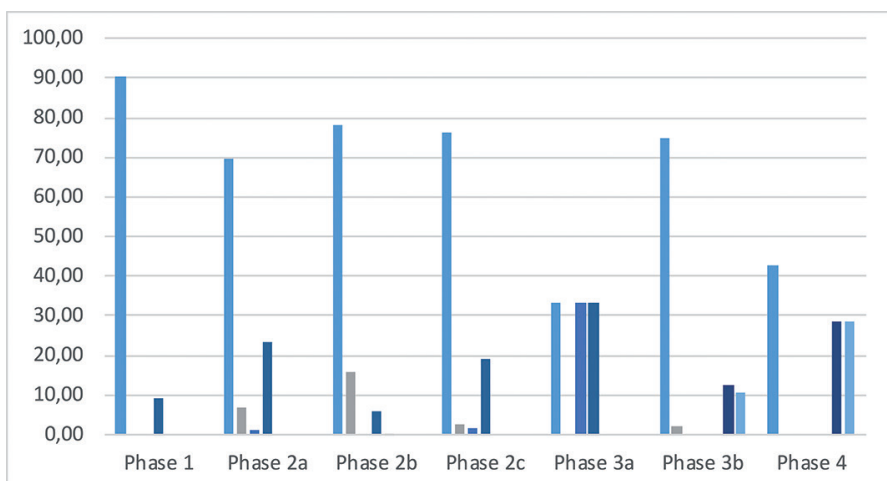

A $\square$ CL-MAS COM-MAS PS-AT GR-MONO AT-VN

A

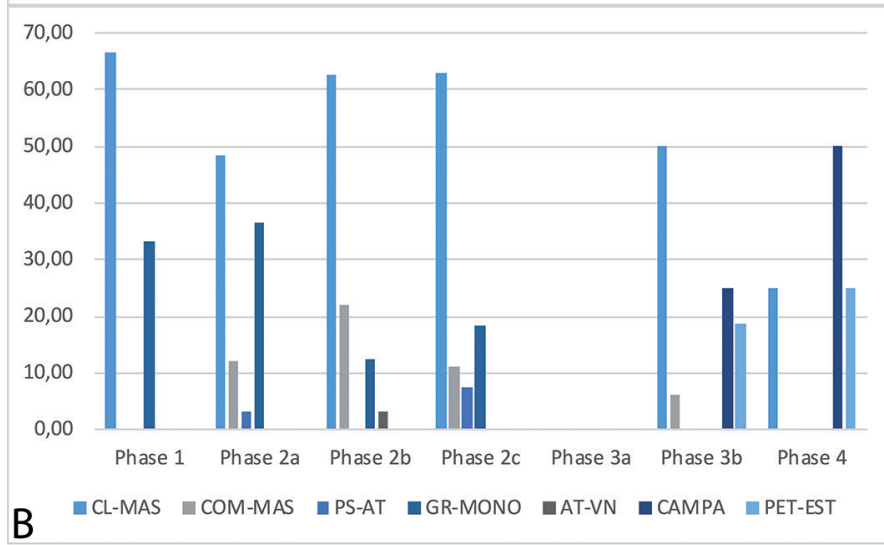

I 52 Proportions des catégories de céramiques tournées.

I A - \% sur total des fragments de céramiques tournées.

I B - \% sur total des individus de céramiques tournées. 
Le répertoire de la céramique tournée utilisée à Martigues apparaît très commun pour les sites de basse Provence occidentale $^{22}$ et conforme aux résultats des études précédentes (Damotte 2003, 216). Peu abondant, il est dominé par les coupes qui représentent plus de $40 \%$ des individus. On trouve ensuite les cruches à pâte claire, avec environ $15 \%$ des individus, auxquelles on peut ajouter les œnochoés en céramique grise monochrome qui ont la même fonction. Les mortiers représentent $14 \%$ des formes tournées. Les autres formes sont marginales, seules quelques olpés devant réellement être prises en compte avec un peu plus de $5 \%$ des formes tournées.

\subsection{Répartition fonction- nelle de la céramique}

\subsubsection{Vaisselle de cuisine}

Cette catégorie apparaît majoritaire avec presque $50 \%$ des individus. C'est notamment grâce à la quantité très importante d'urnes qui représentent plus d'un tiers des formes. L'urne demeure en effet le vase majoritaire de la panoplie de cuisine gauloise. Faites d'abord pour cuire et mijoter les aliments - plusieurs exemplaires présentent des traces de feu sous le fond et sur les flancs - nombreuses sont les urnes à avoir eu d'autres usages. On pense d'une part à la réserve alimentaire pour les plus importantes, en particulier lors des périodes anciennes, et d'autre part à une utilisation comme gobelet ou vase à verser pour les plus petites (fig. 53).

Quant aux jattes, qui représentent $12 \%$ de la vaisselle de cuisine, leurs usages sont également nombreux. Elles permettaient tout autant la préparation de la nourriture, sa cuisson, sa présentation ou encore une consommation en commun (Bats 1988, 219). Celles qui présentent un large verseur réservé sur la lèvre suggèrent évidemment leur utilisation en lien avec des produits liquides ou semi-liquides. On observera surtout leur association fonctionnelle avec les foyers et en particulier avec les fours complexes dont l'un a été mis au jour dans la maison $\mathrm{H} 4$.

Enfin, les mortiers, qui forment les seuls éléments exogènes venant s'insérer dans la vaisselle de cuisine, sont ici assez bien représentés avec environ $11 \%$ des individus de la vaisselle de cuisine. On a là un phénomène bien attesté dans la région de l'étang de Berre qui tranche avec les autres régions de Gaule méridionale où leur part est bien moins importante (Curé 2013, 176) 23. $^{23}$

\begin{tabular}{|c|c|c|c|}
\hline & \multicolumn{3}{|c|}{ Toutes phases } \\
\hline & ind & $\% g r$ & \%tot \\
\hline \multicolumn{4}{|l|}{ Vaisselle de cuisine } \\
\hline \multicolumn{4}{|l|}{ Cuire } \\
\hline Urnes non tournées & 89 & 74,17 & 36,78 \\
\hline \multicolumn{4}{|l|}{ Préparer-Cuire } \\
\hline jattes & 15 & 12,50 & 6,20 \\
\hline \multicolumn{4}{|l|}{ Préparer-broyer } \\
\hline mortiers non tournés & 2 & 1,67 & 0,83 \\
\hline mortiers massaliètes & 14 & 11,67 & 5,79 \\
\hline Total & 120 & 100,00 & 49,59 \\
\hline \multicolumn{4}{|l|}{ Vaisselle de table } \\
\hline \multicolumn{4}{|l|}{ Verser boisson } \\
\hline cruches pâte claire & 16 & 16,00 & 6,61 \\
\hline olpés pâte claire & 7 & 7,00 & 2,89 \\
\hline cruches grises monochromes & 4 & 4,00 & 1,65 \\
\hline \multicolumn{4}{|l|}{ Boire } \\
\hline coupes à deux anses claire & 7 & 7,00 & 2,89 \\
\hline coupes à anse grises monochromes & 3 & 3,00 & 1,24 \\
\hline coupes pseudo-attique & 3 & 3,00 & 1,24 \\
\hline coupes vernis noir & 1 & 1,00 & 0,41 \\
\hline bols campaniens & 4 & 4,00 & 1,65 \\
\hline bols pet-est & 4 & 4,00 & 1,65 \\
\hline \multicolumn{4}{|l|}{ Boire-manger } \\
\hline coupes sans anses pâte claire & 5 & 5,00 & 2,07 \\
\hline coupes carénée pate claire & 3 & 3,00 & 1,24 \\
\hline coupes carénée grise mono & 14 & 14,00 & 5,79 \\
\hline coupes non tournées & 20 & 20,00 & 8,26 \\
\hline coupes monoansées pâte claire & 9 & 9,00 & 3,72 \\
\hline Total & 100 & 100,00 & 41,32 \\
\hline \multicolumn{4}{|l|}{ Divers } \\
\hline indet non tournées & 11 & 50,00 & 4,55 \\
\hline indet pâte claire & 8 & 36,36 & 3,31 \\
\hline indet grises monochromes & 2 & 9,09 & 0,83 \\
\hline askos grise monochrome & 1 & 4,55 & 0,41 \\
\hline Total & 22 & 100,00 & 9,09 \\
\hline Total de la vaisselle & 242 & & \\
\hline
\end{tabular}

I 53 Répartition fonctionnelle de la vaisselle. 


\subsubsection{Vaisselle de table}

La vaisselle de table est constituée de récipients permettant de servir les boissons, de les boire et de manger les aliments (fig. 53). Les cruches sont majoritaires et représentent plus de $20 \%$ des individus de ce groupe (massaliète et grise monochrome $)^{24}$.

En ce qui concerne la consommation des liquides, elle semble être faite principalement dans des formes de céramiques exogènes: des coupes avec ou sans anses massaliètes et en céramique grise monochrome, supplantées par les bols à vernis noir italiens à partir de la fin du $\mathrm{IV}^{\mathrm{e}}$ siècle.

L'introduction puis l'adoption rapide des productions italiques paraissent ici, comme sur bien d'autres habitats protohistoriques, s'intégrer dans des pratiques culinaires déjà existantes, ce qui a fait dire à $M$. Bats qu'il s'agissait de pratiques de substitution de formes plutôt que d'un phénomène d'acculturation (Bats 1988, 223).

\section{Conclusion générale}

Malgré le caractère très particulier et tronqué des recherches réalisées en 1989, les apports de cette campagne sont importants. Elle a d'abord permis de restituer la morphologie générale du premier village gaulois de l'Île de Martigues grâce à la découverte de la partie méridionale du site.

Plusieurs éléments du rempart sud (deux sections de sa courtine, un dispositif d'entrée piétonne au sud-est et le départ d'une tour arrondie au sud-ouest) ont pu être mis au jour ainsi que de nombreux murs appartenant au découpage d'un îlot simple d'habitations, l'îlot $\mathrm{H}$, adossé au rempart qui a pu être partiellement exploré.

Du point de vue topographique et chrono-stratigraphique, les fouilles ont également apporté nombre d'informations nouvelles venant confirmer ou enrichir notre connaissance du site. Au rang des confirmations, l'organisation primitive (périodes 1 et $2 \mathrm{a}$ ) de cette partie sud du village diffère quelque peu de celle adoptée à partir du début du $\mathrm{IV}^{\mathrm{e}}$ s. av. J.-C. (période 2b), comme pour le quartier nordouest. Par le phénomène de remontage systématique des murs sur d'anciennes parois, elles ont aussi montré que le découpage du village perdurait ensuite sans changement pendant deux siècles jusqu'à sa destruction définitive. Autre confirmation, la mise en évidence des mêmes épisodes destructifs qui marquent les périodes $1,2 \mathrm{c}$ et 4 . Les données concernant l'incendie de la période $2 \mathrm{c}$ s'y sont même révélées particulièrement bien documentées, aussi bien du point de vue du mobilier que de celui des aménagements domestiques.

Parmi les éléments nouveaux apportés par cette campagne, on doit signaler, pour les $\mathrm{V}^{\mathrm{e}}$ et $\mathrm{IV}^{\mathrm{e}}$ siècles av. J.-C., la découverte de dépotoirs domestiques et surtout de vestiges d'habitat en dur (murs, foyers) situés hors les murs du village.

Pour ce qui concerne le mobilier céramique, l'intérêt de cette étude est d'offrir la publication d'un corpus homogène et bien daté permettant des comparaisons avec des contextes similaires sur le site même de l'Île ou ailleurs. Le faciès général de la collection étudiée est très proche de celui mis en évidence pour d'autres contextes, les quelques différences relevées étant dues pour l'essentiel à la représentativité parfois insuffisante de notre échantillon. Le faciès céramique de Martigues s'intègre dans celui des autres habitats gaulois de Provence. Pour toutes les périodes, il montre une majorité de céramiques non tournées, avec un répertoire traditionnel des formes, qui n'empêche pas les spécificités propres à la région de l'étang de Berre déjà mises en évidence.

Cependant, à la fois par l'omniprésence de la vaisselle et des amphores massaliètes et par l'émergence de séries particulières de céramique tournée ou d'imitations en pâte locale, on observe ici également l'importance, la variabilité, mais aussi la persistance des liens noués entre la cité grecque et l'agglomération gauloise. C'est à ces divers titres que l'habitat de l'île de Martigues s'impose aujourd'hui comme l'un des principaux sites protohistoriques permettant de comprendre l'évolution des communautés gauloises du Midi durant le second âge du Fer.

AXEL CAUVIN

Doctorant contractuel Aix Marseille Univ, CNRS, Minist Culture, CCJ, Aix-en-Provence, France 
1. Je tiens à remercier chaleureusement J. Chausserie-Laprée de m'avoir confié toute la documentation (photographies, fiches US, dessins et évidemment mobilier céramique) nécessaire à la réalisation de cette étude. Son aide fut précieuse tout au long de la réalisation de ce travail et sans lui et ses nombreuses corrections, cet article n'existerait pas.

2. L'église de la Madeleine, telle qu'on la voit encore aujourd'hui, est un bâtiment imposant, édifié dans la deuxième moitié du XVII' siècle (consécration en 1680) sur des fondations et à la place d'une église antérieure, plus petite, remontant au moins au $X V^{e}$ siècle. C'est à cette première église qu'appartient très probablement le caveau mis au jour. Les recherches effectuées à ce sujet par l'historien René Cornet, que nous remercions ici, relèvent l'inhumation de pas moins de 120 défunts au sein de l'église de La Madeleine entre 1651 et 1653 . Ils représentent près du tiers des 385 défunts relevés dans le registre paroissial des sépultures du quartier de l'île pour ces trois années. Tous les autres, soit 265 individus, ont été enterrés dans le cimetière (ACM, GG 3-2).

3. Deux éléments ont pu être isolés et intégrés dans d'autres travaux, d'une part une amphore étrusque tardive du type A-ETR 4A (Chausserie-Laprée 2004, 51-52, fig. 48, n ${ }^{\circ}$ 5), d'autre part une coupe carénée en céramique grise peinte (type GR-Peinte 4) (Chausserie-Laprée, Nin 1995, 356, fig. 10, $\mathrm{n}^{\mathrm{o}}$ 117). Ces deux objets sont datés des IVe-III' $\mathrm{s}$. av. J.-C.

4. Cette étude est tirée d'une annexe d'un mémoire de Master 2 soutenu en juin 2015 sous la direction de D. Garcia: A. Cauvin, Étude du mobilier des secteurs sud du quartier de l'Île à Martigues, Aix-Marseille Université, 94 p., $17 \mathrm{pl}$.

5. Pour ce qui concerne le sondage 8904 , seules les données qui concernent également l'occupation de l'îlot $\mathrm{H}$ ont été prises en compte.

6. À moins que l'effet de parement multiple de cet amas de blocs ne soit que le résultat de l'effondrement du rempart MR1008. On pourrait le penser au regard de l'absence d'un tel doublage dans le sondage 8904, à l'intérieur à l'église.

7. Le caractère trop incertain des données stratigraphiques de ce secteur nous a fait exclure de cette étude le mobilier en provenant.

8. Cette jatte montre une réparation au moyen d'une agrafe en bronze (Rétif $\left.2000, n^{\circ} 195 a\right)$.

9. L'une, appuyée contre le mur est, repose sur un pied haut de forme quadrangulaire, alors que la seconde parait assise sur un pied bas annulaire.

10. On notera que la fouille exécutée dans la tranchée intérieure à l'église a mis au jour, en relation avec ce niveau de destruction de la maison $\mathrm{H} 3$ (sol 020 du sondage 8904), un fragment de mortier massaliète et une couronne d'argile crue jaune. Ces objets sont associés à deux grandes pierres plates, l'une appartenant à une banquette, l'autre taillée en ovale faisant office de support.

11. On y a trouvé en particulier une fibule et une pointe de trait du type d'Olympie (Rétif 2000, ${ }^{\text {os }} 201$ et 202).

12. Cette tranchée a également oblitéré une bonne part du mur MR1066, non visible dans la coupe, qui forme un refend avec MR1067 et limite la partie sud de la maison mitoyenne $\mathrm{H} 6$.
13. Sur la configuration et le fonctionnement débattu de ces fours, dont on connaît près d'une dizaine d'exemplaires sur le site de l'île, on se reportera à l'étude de N. Nin (2003, 107-113) et aux recherches en cours de Jean Coulon (2015). Leur utilisation a fait l'objet de nombreuses hypothèses, dont l'une, basée sur les fouilles de Philippe Boissinot sur le site de Roquepertuse, évoque leur usage dans le processus de fabrication de la bière (Bouby et al. 2011).

14. La cuisson insuffisante de ces objets en terre crue n'a permis ni de les prélever, ni de les dessiner.

15. Il se distingue en cela des quelques maçonneries très sommaires, de la même époque ( $\mathrm{V}^{\mathrm{e}} \mathrm{I} \mathrm{IV}^{\mathrm{e}} \mathrm{s}$. av. J.-C.) mises au jour au nord-est du premier village lors des fouilles de sauvetage effectuées en 2001 dans la rue de La République.

16. La céramique grise-monochrome, déjà étudiée et détaillée, n'est pas décrite dans cet article; on se réfèrera à la publication de 1995 (Chausserie-Laprée, Nin 1995). Seuls les dessins ont été repris et retravaillés afin de restituer la totalité des ensembles stratigraphiques par période.

17. Cette identification a posteriori fut rendue possible par M. Py que nous remercions ici.

18. Un seul fragment de vaisselle attique à vernis noir, non identifiable, est issu de cette fouille. La coupe de type PSEUDO-AT 349-352 de la période 2a, ne permet pas non plus de resserrer la chronologie étant donné que sa diffusion s'échelonne entre 400 et 300 av. J.-C

19. C'est un pourcentage comparable à ceux mis en évidence au nord-ouest du site: la CNT représente $71,5 \%$ de la vaisselle dans les maisons de l'îlot A étudiées par L. Damotte $(2003,229)$ et encore $61,6 \%$ des contextes de voirie (Chausserie-Laprée, Nin 1995, 341). Une telle concordance confirme la bonne représentativité de la collection étudiée ici.

20. La période 3a ne contient pas assez d'éléments pour que l'on puisse tirer un enseignement des chiffres également importants de doliums.

21. Sur la part des CNT-BER, leur augmentation perçue dans le sondage 8903 , pour la période $2 \mathrm{~b}$ en particulier, est ici plus faible que celle observée au nord-ouest du site (Damotte 2003, Chausserie-Laprée, Nin 1995). C'est probablement dû à la faiblesse de l'échantillon et aussi aux critères d'identification et de discrimination de la vaisselle non tournée en deux groupes (CNTPRO et CNT-BER), qui, selon les chercheurs, aboutissent à son classement dans l'une (CNT-PRO) ou l'autre (CNT-BER) catégorie. Rappelons en effet que la CNT-PRO de Martigues a livré un bon nombre de vases imitant les formes et les décors des céramiques en CNR-BER.

22. À l'exception notable de l'oppidum du Baou de Saint-Marcel qui montre une prévalence de la céramique grise monochrome sur les productions massaliètes à pâte claire (Guichard, Rayssiguier 1993, 247).

23. Sur l'interprétation débattue du mortier comme signe de l'hellénisation des sociétés locales du Midi de la Gaule, voir les travaux d'E. Gomez (2000, 141) à Béziers, et a contrario la réfutation d'A.-M. Curé (2010).

24. Les imitations en céramique locale, marquées par l'existence de vases munis d'anses, ne sont que marginales et relativement tardives (III ${ }^{\mathrm{e}} \mathrm{s}$. av. J.-C.). 
Références bibliographiques

Bats 1988: BATS (M.) - Vaisselle et alimentation à Olbia de Provence (v. 350 - v. 50 av. J.-C.). Modèles culturels et catégories céramiques. Paris, Édition du CNRS, 1988, 272 p. (Revue Archéologique de Narbonnaise, supplément 18).

Barruol, Py 1978 : BARRUOL (G.), PY (M.) - Recherches récentes sur la ville antique d'Espeyran à Saint-Gilles-du-Gard. Revue Archéologique de Narbonnaise, 11, 1978, p. 19-100

Bouby, Boissinot, Marinval 2011 : BOUBY (L.), BOISSINOT (Ph.), MARINVAL (Ph.) - Never Mind the Bottle. Archaeobotanical Evidence of Beer-brewing in Mediterranean France and the Consumption of Alcoholic Beverages During the 5th Century BC. Human Ecology, 39, 2011, p. 351-360.

Campenon, Chausserie-Laprée 2000: CAMPENON (Chr.), CHAUSSERIELAPRÉE (J.) - La céramique attique de Martigues au IVe siècle. In: SABATTINI (B.) dir. - La céramique attique du IV siècle en Méditerranée occidentale. Actes du colloque international organisé par le Centre Camille Jullian, Arles, 7-9 déc. 1995. Naples, Centre Jean Bérard, 2000, p. 145-158.

Chausserie-Laprée 1998: CHAUSSERIE-LAPRÉE (J.) - Les meules des habitats protohistoriques de Martigues. Documents d'Archéologie Méridionale, 21, 1998, p. 211-235.

Chausserie-Laprée 2004 : CHAUSSERIE-LAPRÉE (J.) - La circulation des amphores sur le littoral provençal; l'exemple des habitats gaulois de Martigues. Arqueo Mediterrània, 8, 2004, p. 25-58.

Chausserie-Laprée 2005: CHAUSSERIE-LAPRÉE (J.) - Martigues, terre gauloise entre Celtique et Méditerranée. Paris, Martigues, Errance, Ville de Martigues, 2005, $251 \mathrm{p}$.

Chausserie-Laprée, Nin 1988: CHAUSSERIE-LAPRÉE (J.), NIN (N.) - Le mobilier céramique. Dossiers Histoire et Archéologie., 1988, p. 84-89.

Chausserie-Laprée, Nin 1990 : CHAUSSERIE-LAPRÉE (J.), NIN (N.) - Le village protohistorique du quartier de l'Île à Martigues (Bouches-du-Rhône). Les espaces domestiques de la phase primitive. I - Les aménagements domestiques. Documents d'Archéologie Méridionale, 13, 1990, p. 35-136.

Chausserie-Laprée, Nin 1995 : CHAUSSERIE-LAPRÉE (J.), NIN (N.) - La céramique grise monochrome de l'habitat protohistorique de l'Île à Martigues (Bouches-du-Rhône). In : ARCELIN (P.), BATS (M.), GARCIA (D.), MARCHAND (G.), SCHWALLER (M.) dir. - Sur les pas des Grecs en Occident... : hommages à André Nickels. Paris, Lattes, Errance, A.D.A.M, 1995, p. 339-362, (Études Massaliètes, 4).

Chausserie-Laprée, Domallain, Nin 1984: CHAUSSERIE-LAPRÉE (J.) DOMALLAIN (L.), NIN (N.) - Le quartier de l'Île à Martigues. Six années de recherches archéologiques, Martigues, Musée d'Art et d'Archéologie, $1984,64 \mathrm{p}$

Chazelles, Ugolini 2015 : CHAZELLES (C.-A. de), UGOLINI (D.) dir. - Mont laurès (Narbonne, Aude) à la fin du premier âge du Fer. Lattes, Edition de l'Association pour le Développement de l'archéologie en Languedoc-Roussillon, 2015, $614 \mathrm{p}$

Coulon 2015: COULON (J.) - Les fours dits de «potier» de type Sévrier (Haute-Savoie, France). Les indices d'une hypothèse fonctionnelle alternative. In : BOULUD-GAZO (S.), NICOLAS (Th.) dir. - Artisanats et productions à l'âge du Bronze. Dijon, Paris, Association pour la promotion des recherches sur l'âge du Bronze, Société préhistorique française, 2015, p. 109-122. (Séances de la Société préhistorique française, 4).
Curé 2010 : CURÉ (A.-M.) - Réflexions sur l'utilisation culinaire des mortiers protohistoriques en céramique: Le cas du Languedoc méditerranéen à l'âge du Fer (VIe-III e av. n. è.) In : Mata Parreño (C.), Pérez Jordá (G.), Vives-Ferrándiz Sánchez (J.) dir. - De la cuina a la taula. IV reunio d'economia en el premier mil.lenni aC. 2010, p. 189-198. (Saguntum, supplément 9).

Curé 2013 : CURÉ (A.-M.) - La céramique de cuisine tournée et les pratiques culinaires à l'âge du Fer en Gaule méditerranéenne. Thèse de doct., Montpellier: Université Paul Valéry 3, 2013, 386 p.

Damotte 2003 : DAMOTTE (L.) - Mobilier céramique et faciès culturel de l'habitat gaulois de l'Île de Martigues. Documents d'Archéologie Méridionale, 26, 2003, p. 171-234.

Duval 1998: DUVAL (S.) - Bilan de recherches et d'études du mobilier des fouilles de Charles Lagrand. Documents d'Archéologie Méridionale, 21, 1998, p. 133-180.

Girard-Millereau 2010: GIRARD-MILLEREAU (B.) - Le mobilier métallique de l'âge du Fer en Provence (VIe - I ${ }^{e r}$ s. av. J.-C.). Contribution à l'étude des Celtes de France méditerranéenne. Thèse de doct., Dijon, Aix-en-Provence: Université de Bourgogne, Université de Provence, 2010, 722 p.

Gomez 2000 : GOMEZ (É.) - Contribution à l'étude des mortiers de cuisine : les mortiers du Languedoc occidental du VIe au IVe s. av. J.-C. Documents d'Archéologie Méridionale, 23, 2000, p. 113-143.

Guichard, Rayssiguier 1993 : GUICHARD (Chr.), RAYSSIGUIER (G.) - Les Baou-de Saint-Marcel à Marseille. Étude stratigraphique du secteur III (VI ${ }^{\mathrm{e}}$-II ${ }^{\mathrm{e}}$ siècles avant J.-C.). Documents d'Archéologie Méridionale, 16, 1993, p. 231-256.

Legros 1986: LEGROS (Th.) - Les Salins de Ferrières et l'Abion à Martigues (B.-du-Rh.) Cahiers Ligures de Préhistoire et de Protohistoire, nouvelle série, 3. 1986. p. 227-257.

Marino, Damotte, Mercurin 2009: MARINO (H.), DAMOTTE (L.), MERCURIN (R.) - Martigues, Chapelle de l'Annonciade, B.S.R.-PACA, 2008, p. 147-149

Marty 2002 : MARTY (Fr.) - L'habitat de hauteur du Castellan (Istres, Bouchesdu-Rhône) à l'âge du Fer. Étude des collections anciennes et recherches récentes. Documents d'Archéologie Méridionale, 25, 2002, p. 129-169.

Marty 2005: MARTY (Fr.) - La céramique non tournée domestique de l'âge du Fer en Provence. Mémoire de Master de Recherche en Archéologie et Histoire de l'Art. Aix-en-Provence: Université de Provence - Aix-Marseille 1, $2005,144 \mathrm{p}$.

Morel 1965: MOREL (J.-P.) - Céramique à vernis noir du forum romain et $d u$ Palatin. Paris, De Boccard, 1965, 274 p.

Morel 1969: MOREL (J.-P.) - Études de céramique campanienne, I: l'atelier des petites estampilles. Mélanges d'archéologie et d'histoire, 81, 1969, p. 59-117.

Moulin 2014: MOULIN (C.) - La céramique peinte à pâte claire du Pègue (Drôme) : caractérisation et répartition. Mémoire de Master 2, Université Lyon 2, 2014, 203 p.

Nin 1999: NIN (N.) - Les espaces domestiques en Provence durant la Protohistoire. Aménagements et pratiques rituelles du $\mathrm{VI}^{\mathrm{e}} \mathrm{s}$. av. n. è. à l'époque augustéenne. Documents d'Archéologie Méridionale, 22, 1999, p. 221-278. 
Nin 2003: NIN (N.) - Vases et objets en terre crue dans le Midi durant l'Âge du Fer. In: CHAZELLES (C.-A. de), KLEIN (A.) dir. - Échanges transdisciplinaires sur les constructions en terre crue, 1. Table ronde de Montpellier, 17-18 novembre 2001. Montpellier, Édition de l’Espérou, 2003, p. 95-146.

Nin, Chausserie-Laprée 2016: NIN (N.), CHAUSSERIE-LAPRÉE (J.) - Les fosses rituelles de l'oppidum de Saint-Pierre-les-Martigues: mythes ou réalité. Les données des fouilles de Charles Lagrand. In: CHAZELLES (C.-A. de), SCHWALLER (M.) dir. - Vie quotidienne, tombes et symboles des sociétés protohistoriques de Méditerranée nord-occidentale. Lattes, Publication de l'UMR 5140 du CNRS «Archéologie des Sociétés Méditerranéennes», Éditions de l'Association pour le Développement de l'Archéologie en Languedoc-Roussillon, 2016, p. 677-702. (Monographies d'Archéologie Méditerranéenne. Hors-série 7).

Py et al. 1993: PY (M.), ADROHER AUROUX (A. M.), RAYNAUD (Cl.) dir. Dicocer. Dictionnaire des céramiques antiques (VII s. av. n. è.-VII s. de n. è.) en Méditerranée nord-occidentale (Provence, Languedoc, Ampurdan). Lattes, Édition de l'Association pour la Recherche Archéologique en Languedoc Oriental, 1993, 622 p. (Lattara, 6).

Py et al. 2001 : PY (M.), ADROHER AUROUX (A. M.), SANCHEZ (C.) - Dicocer 2. Corpus des céramiques de l'âge du Fer de Lattes (fouilles 1963-1999) - Tome 1 et 2. Lattes, Édition de 1'Association pour la Recherche Archéologique en Languedoc Oriental, 2001, 1305 p. (Lattara, 14).

Py 2006: PY (M.) - La zone H de l'oppidum des Castels (Nages-et-Solorgues, Gard). Documents d'Archéologie Méridionale, 29-30, 2006-2007, p. 277-344.
Py 2016: PY (M.) - Dictionnaire des objets protohistoriques de Gaule méditerranéenne ( $\left(X^{e}-I^{e r}\right.$ siècles avant notre ère). Lattes, Édition de l'Association pour la Recherche Archéologique en Languedoc Oriental, 2016, 576 p. (Lattara, 23).

Ratsimba 2005: RATSIMBA (A.) - Le pithos en Gaule méridionale: Production, diffusion et utilisation d'un mobilier d'origine grecque (VI'-IVE s. av. J.-C.). Mémoire de Master de Recherche en Archéologie et Histoire de l'Art, Aixen-Provence, Université de Provence, 2005, 73 p.

Raux 1999: RAUX (St.) - Les objets de la vie quotidienne à Lattes au IVe siècle avant notre ère. In: PY (M.) dir. - Recherches sur le quatrième siècle avant notre ère à Lattes. Lattes, Édition de l'Association pour la Recherche Archéologique en Languedoc Oriental, 1999, p. 439-518. (Lattara, 12).

Rétif 2000: RÉTIF (M.) - Le mobilier métallique de l'habitat protohistorique de l'Île de Martigues, Bouches-du-Rhône (Ve-II $\mathrm{e}$. av. J.-C.). Documents d'Archéologie Méridionale, 23, 2000, p. 157-208.

Sanmartí-Grego 1973: SANMARTI-GREGO (E.) - El taller de las pequeñas estampillas en la Península Ibérica. Ampurias, 35, 1973, p. 135-173.

Savarese 2011 : SAVARESE (L.) - Les marques sur amphores découvertes dans les Pyrénées-Orientales. Sylloge Epigraphica Barcinonensis, 9, 2011, p. 207-269

Verdin et al. 1996-1997: VERDIN (Fl.), BRIEN-POITEVIN (Fr.), CHABAL (L.), MARINVAL (Ph.), PROVANSAL (M.) - Coudounèu (Lançon-Provence, Bouches-du-Rhône) : une ferme grenier et son terroir au $\mathrm{V}^{\mathrm{e}}$ siècle avant J.-C. Documents d'Archéologie Méridionale, 19-20, 1996-1997, p. 165-198. 